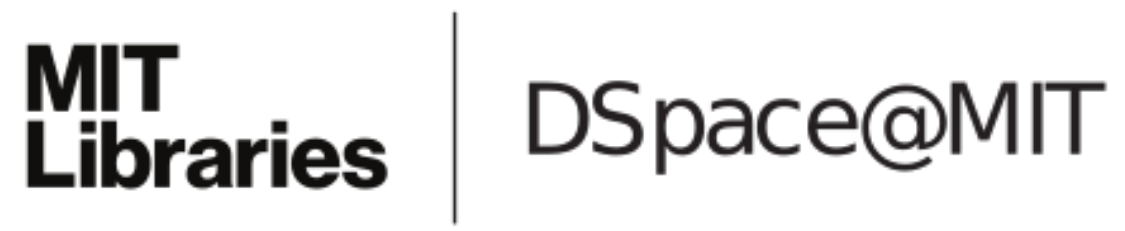

\author{
MIT Open Access Articles
}

Higgs boson studies at the Tevatron

The MIT Faculty has made this article openly available. Please share how this access benefits you. Your story matters.

Citation: Aaltonen, T., V. M. Abazov, B. Abbott, B. S. Acharya, M. Adams, T. Adams, J. P. Agnew, et al. “Higgs boson studies at the Tevatron." Physical Review D 88, no. 5 (September 2013). (C) 2013 American Physical Society

As Published: http://dx.doi.org/10.1103/PhysRevD.88.052014

Publisher: American Physical Society

Persistent URL: http://hdl.handle.net/1721.1/84685

Version: Final published version: final published article, as it appeared in a journal, conference proceedings, or other formally published context

Terms of Use: Article is made available in accordance with the publisher's policy and may be subject to US copyright law. Please refer to the publisher's site for terms of use. 


\section{Higgs boson studies at the Tevatron}

T. Aaltonen, ${ }^{12, *}$ V. M. Abazov, ${ }^{47, \dagger}$ B. Abbott,${ }^{109, \dagger}$ B. S. Acharya,${ }^{30, \dagger}$ M. Adams,${ }^{77, \dagger}$ T. Adams, ${ }^{73, \dagger}$ J. P. Agnew, ${ }^{64, \dagger}$ G. D. Alexeev,${ }^{47, \dagger}$ G. Alkhazov ${ }^{51, \dagger}$ A. Alton,${ }^{93, \dagger, \text { ii }}$ S. Amerio, ${ }^{34 a, *}$ D. Amidei,${ }^{93, *}$ A. Anastassov,${ }^{75, *, c}$ A. Annovi,${ }^{33, *}$ J. Antos ${ }^{52, *}$ G. Apollinari ${ }^{75, *}$ J. A. Appel,${ }^{75, *}$ T. Arisawa, ${ }^{40, *}$ A. Artikov,${ }^{47, *}$ J. Asaadi,${ }^{116, *}$ W. Ashmanskas, ${ }^{75, *}$ A. Askew, ${ }^{73, \dagger}$ S. Atkins, ${ }^{87, \dagger}$ B. Auerbach,${ }^{74, *}$ K. Augsten, ${ }^{9, \dagger}$ A. Aurisano, ${ }^{116, *}$ C. Avila, ${ }^{7, \dagger}$ F. Azfar, ${ }^{65, *}$ F. Badaud ${ }^{13, \dagger}$ W. Badgett ${ }^{75, *}$ T. Bae,${ }^{42, *}$ L. Bagby,${ }^{75, \dagger}$ B. Baldin, ${ }^{75, \dagger}$ D. V. Bandurin, ${ }^{73, \dagger}$ S. Banerjee, ${ }^{30, \dagger}$ A. Barbaro-Galtieri, ${ }^{67, *}$

E. Barberis, ${ }^{89, \dagger}$ P. Baringer, ${ }^{86, \dagger}$ V. E. Barnes, ${ }^{84, *}$ B. A. Barnett, ${ }^{88, *}$ P. Barria,${ }^{35 c, 35 a, *}$ J. F. Bartlett,${ }^{75, \dagger}$ P. Bartos, ${ }^{52, *}$ U. Bassler, ${ }^{18, \dagger}$ M. Bauce, ${ }^{34 b, 34 a, *}$ V. Bazterra, ${ }^{77, \dagger}$ A. Bean ${ }^{86, \dagger}$ F. Bedeschi, ${ }^{35 a, *}$ M. Begalli, ${ }^{2, \dagger}$ S. Behari, ${ }^{75, *}$ L. Bellantoni, ${ }^{75, \dagger}$ G. Bellettini, ${ }^{35 b, 35 a, *}$ J. Bellinger, ${ }^{122, *}$ D. Benjamin, ${ }^{106, *}$ A. Beretvas,${ }^{75, *}$ S. B. Beri, ${ }^{28, \dagger}$ G. Bernardi, ${ }^{17, \dagger}$ R. Bernhard, ${ }^{22, \dagger}$

I. Bertram, ${ }^{60, \dagger}$ M. Besançon, ${ }^{18, \dagger}$ R. Beuselinck, ${ }^{62, \dagger}$ P. C. Bhat,${ }^{75, \dagger}$ S. Bhatia,${ }^{96, \dagger}$ V. Bhatnagar, ${ }^{28, \dagger}$ A. Bhatti, ${ }^{102, *}$

K. R. Bland,${ }^{119, *}$ G. Blazey, ${ }^{78, \dagger}$ S. Blessing, ${ }^{73, \dagger}$ K. Bloom,${ }^{97, \dagger}$ B. Blumenfeld,${ }^{88, *}$ A. Bocci, ${ }^{106, *}$ A. Bodek,${ }^{103, *}$ A. Boehnlein ${ }^{75, \dagger}$ D. Boline,${ }^{104, \dagger}$ E. E. Boos,${ }^{49, \dagger}$ G. Borissov,${ }^{60, \dagger}$ D. Bortoletto, ${ }^{84, *}$ J. Boudreau, ${ }^{113, *}$ A. Boveia ${ }^{76, *}$

A. Brandt, ${ }^{115, \dagger}$ O. Brandt, ${ }^{23, \dagger}$ L. Brigliadori, ${ }^{32 b, 32 a, *}$ R. Brock, ${ }^{95, \dagger}$ C. Bromberg, ${ }^{95, *}$ A. Bross, ${ }^{75, \dagger}$ D. Brown, ${ }^{17, \dagger}$ E. Brucken, ${ }^{12, *}$ J. Budagov, ${ }^{47, *}$ X. B. Bu, ${ }^{75, \dagger}$ H. S. Budd, ${ }^{103, *}$ M. Buehler,${ }^{75}{ }^{\dagger}$ V. Buescher, ${ }^{25, \dagger}$ V. Bunichev,${ }^{49, \dagger}$ S. Burdin, ${ }^{60, \dagger, j j}$ K. Burkett, ${ }^{75, *}$ G. Busetto, ${ }^{34 b, 34 a, *}$ P. Bussey, ${ }^{59, *}$ C. P. Buszello, ${ }^{57, \dagger}$ P. Butti, ${ }^{35 b, 35 a, *}$ A. Buzatu, ${ }^{59, *}$ A. Calamba, ${ }^{12, *}$ E. Camacho-Pérez,${ }^{44, \dagger}$ S. Camarda, ${ }^{53, *}$ M. Campanelli, ${ }^{63, *}$ F. Canelli, ${ }^{76, *, c c}$ B. Carls,${ }^{80, *}$ D. Carlsmith ${ }^{122, *}$ R. Carosi, ${ }^{35 a, *}$ S. Carrillo, ${ }^{72, *, \mathrm{~d}}$ B. Casal,${ }^{56, *, \mathrm{e}}$ M. Casarsa, ${ }^{37 \mathrm{a}, *}$ B. C. K. Casey,${ }^{75, \dagger}$ H. Castilla-Valdez, ${ }^{44, \dagger}$ A. Castro, ${ }^{32 \mathrm{~b}, 32 \mathrm{a}}$ P. Catastini, ${ }^{90, *}$ S. Caughron, ${ }^{95, \dagger}$ D. Cauz,${ }^{37 a *}$ V. Cavaliere, ${ }^{80, *}$ M. Cavalli-Sforza, ${ }^{53, *}$ A. Cerri, ${ }^{67, *, f}$ L. Cerrito, ${ }^{63,9}$ S. Chakrabarti, ${ }^{104, \dagger}$

K. M. Chan,${ }^{83, \dagger}$ A. Chandra, ${ }^{118, \dagger}$ E. Chapon,${ }^{18, \dagger}$ G. Chen,${ }^{86, \dagger}$ Y.C. Chen, ${ }^{6, *}$ M. Chertok, ${ }^{68, *}$ G. Chiarelli, ${ }^{35 a, *}$

G. Chlachidze, ${ }^{75, *}$ K. Cho, ${ }^{42, *}$ S. W. Cho, ${ }^{43, \dagger}$ S. Choi, ${ }^{43, \dagger}$ D. Chokheli, ${ }^{47, *}$ B. Choudhary, ${ }^{29, \dagger}$ S. Cihangir, ${ }^{75, \dagger}$

M. A. Ciocci, ${ }^{35 c, 35 a, *}$ D. Claes, ${ }^{97, \dagger}$ A. Clark,${ }^{58, *}$ C. Clarke,,${ }^{94, *}$ J. Clutter, ${ }^{86, \dagger}$ M. E. Convery,${ }^{75, *}$ J. Conway, ${ }^{68, *}$ M. Cooke, ${ }^{75, \dagger}$ W. E. Cooper, ${ }^{75 \dagger}$ M. Corbo,${ }^{75, *}$ M. Corcoran, ${ }^{118, \dagger}$ M. Cordelli, ${ }^{33, *}$ F. Couderc,,${ }^{18, \dagger}$ M.-C. Cousinou, ${ }^{15, \dagger}$ C. A. Cox,${ }^{68, *}$ D. J. Cox,${ }^{68, *}$ M. Cremonesi, ${ }^{35 a, *}$ D. Cruz, ${ }^{16, *}$ J. Cuevas, ${ }^{56, *, b}$ R. Culbertson, ${ }^{75, *}$ D. Cutts, ${ }^{114, \dagger}$ N. d'Ascenzo, ${ }^{75, *, \mathrm{~h}}$ A. Das, ${ }^{66, \dagger}$ M. Datta,${ }^{75, *, d d}$ G. Davies ${ }^{62, \dagger}$ P. De Barbaro, ${ }^{103, *}$ S. J. de Jong,${ }^{45,46, \dagger}$ E. De La Cruz-Burelo, ${ }^{44, \dagger}$ F. Déliot, ${ }^{18, \dagger}$ R. Demina,${ }^{103, \dagger}$ L. Demortier, ${ }^{102, *}$ M. Deninno, ${ }^{32 a, *}$ D. Denisov, ${ }^{75, \dagger}$ S. P. Denisov,${ }^{50, \dagger}$ M. d'Errico, ${ }^{34 b, 34 a, *}$ S. Desai, ${ }^{75, \dagger}$ C. Deterre ${ }^{23, \dagger, 11}$ K. DeVaughan, ${ }^{97, \dagger}$ F. Devoto, ${ }^{12, *}$ A. Di Canto, ${ }^{35 b, 35 a, *}$ B. Di Ruzza, ${ }^{75, *, e e}$ H. T. Diehl,${ }^{75, \dagger}$ M. Diesburg, ${ }^{75, \dagger}$ P. F. Ding, ${ }^{64, \dagger}$ J. R. Dittmann, ${ }^{119, *}$ A. Dominguez,${ }^{97, \dagger}$ S. Donati, ${ }^{35 b, 35 a, *}$ M. D’Onofrio,${ }^{61, *}$ M. Dorigo, ${ }^{37 b, 37 a, *}$ A. Driutti, ${ }^{37 a, *}$ A. Dubey,${ }^{29, \dagger}$ L. V. Dudko, ${ }^{49, \dagger}$ A. Duperrin, ${ }^{15, \dagger}$ S. Dutt,${ }^{28, \dagger}$ M. Eads,${ }^{78, \dagger}$ K. Ebina, ${ }^{40, *}$ R. Edgar, ${ }^{93, *}$ D. Edmunds, ${ }^{95, \dagger}$ A. Elagin,${ }^{16, *}$ J. Ellison, ${ }^{70, \dagger}$ V.D. Elvira ${ }^{75, \dagger}$ Y. Enari, ${ }^{17, \dagger}$ R. Erbacher ${ }^{68, *}$ S. Errede,${ }^{80, *}$ B. Esham, ${ }^{80, *}$ R. Eusebi, ${ }^{116, *}$

H. Evans ${ }^{81, \dagger}$ V. N. Evdokimov, ${ }^{50, \dagger}$ G. Facini, ${ }^{89, \dagger}$ S. Farrington, ${ }^{65, *}$ A. Fauré, ${ }^{18, \dagger}$ L. Feng, ${ }^{78, \dagger}$ T. Ferbel,,${ }^{103, \dagger}$

J.P. Fernández Ramos, ${ }^{55, *}$ F. Fiedler ${ }^{25, \dagger}$ R. Field ${ }^{72, *}$ F. Filthaut, ${ }^{45,46, \dagger}$ W. Fisher,${ }^{95, \dagger}$ H. E. Fisk, ${ }^{75, \dagger}$ G. Flanagan, ${ }^{75, *, j}$ R. Forrest,${ }^{68, *}$ M. Fortner, ${ }^{78, \dagger}$ H. Fox,${ }^{60, \dagger}$ M. Franklin,,${ }^{90, *}$ J. C. Freeman, ${ }^{75, *}$ H. Frisch, ${ }^{76, *}$ S. Fuess, ${ }^{75, \dagger}$ Y. Funakoshi, ${ }^{40, *}$

A. Garcia-Bellido, ${ }^{103, \dagger}$ J. A. García-González, ${ }^{44, \dagger}$ A. F. Garfinkel, ${ }^{84, *}$ P. Garosi,${ }^{35 c, 35 a, *}$ V. Gavrilov, ${ }^{48, \dagger}$ W. Geng, ${ }^{15,95, \dagger}$ C. E. Gerber, ${ }^{77, \dagger}$ H. Gerberich, ${ }^{80, *}$ E. Gerchtein, ${ }^{75, *}$ Y. Gershtein,${ }^{99,}$ S. Giagu, ${ }^{36 a, *}$ V. Giakoumopoulou ${ }^{27, *}$ K. Gibson, ${ }^{113, *}$ C. M. Ginsburg ${ }^{75, *}$ G. Ginther, ${ }^{75,103, \dagger}$ N. Giokaris,${ }^{27, *}$ P. Giromini, ${ }^{33, *}$ G. Giurgiu, ${ }^{88, *}$ V. Glagolev, ${ }^{47, *}$ D. Glenzinski, ${ }^{75, *}$

M. Gold, ${ }^{100, *}$ D. Goldin, ${ }^{116, *}$ A. Golossanov, ${ }^{75}, *$ G. Golovanov, ${ }^{47, \dagger}$ G. Gomez,${ }^{56, *}$ G. Gomez-Ceballos, ${ }^{91, *}$

M. Goncharov, ${ }^{91, *}$ O. González López, ${ }^{55 *}$ I. Gorelov, ${ }^{100, *}$ A. T. Goshaw, ${ }^{106, *}$ K. Goulianos, ${ }^{102, *}$ E. Gramellini, ${ }^{32 a, *}$

P.D. Grannis, ${ }^{104, \dagger}$ S. Greder,${ }^{19, \dagger}$ H. Greenlee, ${ }^{75, \dagger}$ G. Grenier,${ }^{20, \dagger}$ S. Grinstein, ${ }^{53, *}$ Ph. Gris, ${ }^{13, \dagger}$ J.-F. Grivaz, ${ }^{16, \dagger}$

A. Grohsjean, ${ }^{18, \dagger, 11}$ C. Grosso-Pilcher ${ }^{76, *}$ R. C. Group ${ }^{120,75, *}$ S. Grünendahl, ${ }^{75, \dagger}$ M. W. Grünewald, ${ }^{31, \dagger}$ T. Guillemin, ${ }^{16, \dagger}$

J. Guimaraes da Costa, ${ }^{90, *}$ G. Gutierrez, ${ }^{75, \dagger}$ P. Gutierrez, ${ }^{109, \dagger}$ S. R. Hahn, ${ }^{75, *}$ J. Haley, ${ }^{89, \dagger}$ J. Y. Han, ${ }^{103, *}$ L. Han, ${ }^{5, \dagger}$

F. Happacher ${ }^{33, *}$ K. Hara, ${ }^{41, *}$ K. Harder,${ }^{64, \dagger}$ M. Hare, ${ }^{92, *}$ A. Harel, ${ }^{103, \dagger}$ R. F. Harr, ${ }^{94, *}$ T. Harrington-Taber, ${ }^{75, *, s}$ K. Hatakeyama, ${ }^{119, *}$ J. M. Hauptman,${ }^{85, \dagger}$ C. Hays,${ }^{65, *}$ J. Hays,${ }^{62, \dagger}$ T. Head,${ }^{64, \dagger}$ T. Hebbeker,${ }^{21, \dagger}$ D. Hedin,${ }^{78, \dagger}$ H. Hegab, ${ }^{110, \dagger}$ J. Heinrich, ${ }^{111, *}$ A. P. Heinson,${ }^{70, \dagger}$ U. Heintz, ${ }^{114, \dagger}$ C. Hensel,${ }^{23, \dagger}$ I. Heredia-De La Cruz, ${ }^{44, \dagger, k \mathrm{k}}$ M. Herndon, ${ }^{122, *}$ K. Herner, ${ }^{75, \dagger}$ G. Hesketh ${ }^{64, \dagger, m m}$ M. D. Hildreth, ${ }^{83, \dagger}$ R. Hirosky, ${ }^{120, \dagger}$ T. Hoang, ${ }^{73, \dagger}$ J. D. Hobbs, ${ }^{104, \dagger}$ A. Hocker, ${ }^{75, *}$ B. Hoeneisen, ${ }^{11, \dagger}$ J. Hogan,${ }^{18, \dagger}$ M. Hohlfeld,${ }^{25, \dagger}$ J. L. Holzbauer, ${ }^{96, \dagger}$ Z. Hong,${ }^{116, *}$ W. Hopkins,${ }^{75, *, k}$ S. Hou, ${ }^{6, *}$ I. Howley, ${ }^{115, \dagger}$ Z. Hubacek, ${ }^{9,18, \dagger}$

R. E. Hughes, ${ }^{107, *}$ U. Husemann, ${ }^{71, *}$ M. Hussein, ${ }^{95, *, i}$ J. Huston, ${ }^{95, *}$ V. Hynek, ${ }^{9, \dagger}$ I. Iashvili, ${ }^{101, \dagger}$ Y. Ilchenko, ${ }^{117, \dagger}$

R. Illingworth, ${ }^{75, \dagger}$ G. Introzzi,${ }^{35 e, 35 a, *}$ M. Iori, ${ }^{36 b, 36 a, *}$ A. S. Ito ${ }^{75, \dagger}$ A. Ivanov,${ }^{68, *, 1}$ S. Jabeen, ${ }^{114, \dagger}$ M. Jaffré,${ }^{16, \dagger}$ E. James, ${ }^{75, *}$ D. Jang, ${ }^{112, *}$ A. Jayasinghe, ${ }^{109, \dagger}$ B. Jayatilaka, ${ }^{75, *}$ E. J. Jeon, ${ }^{42, *}$ M. S. Jeong, ${ }^{43, \dagger}$ R. Jesik, ${ }^{62, \dagger}$ P. Jiang, ${ }^{5, \dagger}$ S. Jindariani ${ }^{75, *}$

K. Johns, ${ }^{66, \dagger}$ E. Johnson, ${ }^{95, \dagger}$ M. Johnson, ${ }^{75, \dagger}$ A. Jonckheere, ${ }^{75, \dagger}$ M. Jones, ${ }^{84, *}$ P. Jonsson, ${ }^{62, \dagger}$ K. K. Joo, ${ }^{42, *}$ J. Joshi, ${ }^{70, \dagger}$ S. Y. Jun, ${ }^{112, *}$ A. W. Jung, ${ }^{75, \dagger}$ T. R. Junk,${ }^{75, *}$ A. Juste, ${ }^{54, \dagger}$ E. Kajfasz, ${ }^{15, \dagger}$ M. Kambeitz ${ }^{24, *}$ T. Kamon, ${ }^{42,116, *}$ 
P. E. Karchin, ${ }^{94, *}$ D. Karmanov ${ }^{49, \dagger}$ A. Kasmi,${ }^{119, *}$ Y. Kato,${ }^{39, * m}$ I. Katsanos,${ }^{97, \dagger}$ R. Kehoe, ${ }^{117, \dagger}$ S. Kermiche ${ }^{15, \dagger}$

W. Ketchum, ${ }^{76, *, f f}$ J. Keung, ${ }^{111, *}$ N. Khalatyan, ${ }^{75, \dagger}$ A. Khanov, ${ }^{110, \dagger}$ A. Kharchilava, ${ }^{101, \dagger}$ Y. N. Kharzheev, ${ }^{47, \dagger}$

B. Kilminster, ${ }^{75, * \text { cc }}$ D. H. Kim, ${ }^{42, *}$ H. S. Kim,${ }^{42, *}$ J. E. Kim ${ }^{42, *}$ M. J. Kim,${ }^{33, *}$ S. B. Kim, ${ }^{42, *}$ S. H. Kim, ${ }^{41, *}$ Y. J. Kim, ${ }^{42, *}$

Y. K. Kim, ${ }^{76, *}$ N. Kimura, ${ }^{40, *}$ M. Kirby, ${ }^{75, *}$ I. Kiselevich, ${ }^{48, \dagger}$ K. Knoepfel,,${ }^{75, *}$ J. M. Kohli, ${ }^{28, \dagger}$ K. Kondo, ${ }^{40, *, a}$

D. J. Kong, ${ }^{42, *}$ J. Konigsberg, ${ }^{72, *}$ A. V. Kotwal, ${ }^{106, *}$ A. V. Kozelov, ${ }^{50, \dagger}$ J. Kraus,${ }^{96, \dagger}$ M. Kreps,${ }^{24, *}$ J. Kroll, ${ }^{111, *}$ M. Kruse, ${ }^{106, *}$ T. Kuhr,${ }^{24, *}$ A. Kumar, ${ }^{101, \dagger}$ A. Kupco, ${ }^{10, \dagger}$ M. Kurata,${ }^{41, *}$ T. Kurča,${ }^{20, \dagger}$ V. A. Kuzmin, ${ }^{49, \dagger}$ A. T. Laasanen, ${ }^{84, *}$ S. Lammel, ${ }^{75, *}$ S. Lammers,${ }^{81, \dagger}$ M. Lancaster, ${ }^{63, *}$ K. Lannon, ${ }^{107, *, n}$ G. Latino, ${ }^{35 c, 35 a, *}$ P. Lebrun, ${ }^{20, \dagger}$ H. S. Lee, ${ }^{43, \dagger}$ H. S. Lee, ${ }^{42, *}$ J. S. Lee, ${ }^{42, *}$ S. W. Lee,${ }^{85, \dagger}$ W. M. Lee,${ }^{73, \dagger}$ X. Lei,${ }^{66, \dagger}$ J. Lellouch,${ }^{17, \dagger}$ S. Leo, ${ }^{35 a, *}$ S. Leone, ${ }^{35 a, *}$ J. D. Lewis, ${ }^{75, *}$ D. Li ${ }^{17, \dagger}$ H. Li,${ }^{120, \dagger}$ L. Li ${ }^{70, \dagger}$ Q. Z. Li ${ }^{75, \dagger}$ J. K. Lim ${ }^{43, \dagger}$ A. Limosani, ${ }^{106, *, o}$ D. Lincoln, ${ }^{75, \dagger}$ J. Linnemann, ${ }^{95, \dagger}$ V. V. Lipaev, ${ }^{50, \dagger}$ E. Lipeles, ${ }^{111, *}$ R. Lipton,${ }^{75, \dagger}$ A. Lister, ${ }^{58, *, b b}$ H. Liu, ${ }^{120, *}$ H. Liu, ${ }^{117, \dagger}$ Q. Liu, ${ }^{84, *}$ T. Liu ${ }^{75, *}$ Y. Liu, ${ }^{5, \dagger}$ A. Lobodenko, ${ }^{51, \dagger}$ S. Lockwitz, ${ }^{71, *}$ A. Loginov, ${ }^{71, *}$ M. Lokajicek, ${ }^{10, \dagger}$ R. Lopes de Sa, ${ }^{104, \dagger}$ D. Lucchesi, ${ }^{34 b, 34 a, *}$ J. Lueck, ${ }^{24, *}$ P. Lujan, ${ }^{67, *}$ P. Lukens, ${ }^{75, *}$ R. Luna-Garcia, ${ }^{44, \dagger, n n}$ G. Lungu, ${ }^{102, *}$ A. L. Lyon, ${ }^{75, \dagger}$ J. Lys, ${ }^{67, *}$ R. Lysak, ${ }^{52, *, p}$ A. K. A. Maciel, ${ }^{1, \dagger}$ R. Madar, ${ }^{22, \dagger}$ R. Madrak, ${ }^{75, *}$ P. Maestro, ${ }^{35 c, 35 a, *}$ R. Magaña-Villalba, ${ }^{44, \dagger}$ S. Malik, ${ }^{102, *}$ S. Malik, ${ }^{97, \dagger}$ V. L. Malyshev, ${ }^{47, \dagger}$ G. Manca, ${ }^{61, *, \mathrm{q}}$ A. Manousakis-Katsikakis, ${ }^{27, *}$ J. Mansour, ${ }^{23, \dagger}$ F. Margaroli, ${ }^{36 a, *}$ P. Marino, ${ }^{35 \mathrm{~d}, 35 \mathrm{3}, *}$ M. Martínez ${ }^{53, *}$ J. Martínez-Ortega ${ }^{44, \dagger}$ K. Matera,${ }^{80, *}$ M.E. Mattson, ${ }^{94, *}$ A. Mazzacane, ${ }^{75, *}$ P. Mazzanti, ${ }^{32 a, *}$ R. McCarthy, ${ }^{104, \dagger}$ C. L. McGivern,${ }^{64, \dagger}$ R. McNulty,${ }^{61, *, r}$ A. Mehta, ${ }^{61, *}$ P. Mehtala, ${ }^{12, *}$ M. M. Meijer, ${ }^{45,46, \dagger}$ A. Melnitchouk ${ }^{75, \dagger}$ D. Menezes, ${ }^{78, \dagger}$ P. G. Mercadante,${ }^{3, \dagger}$ M. Merkin,${ }^{49, \dagger}$ C. Mesropian, ${ }^{102, *}$ A. Meyer, ${ }^{21, \dagger}$ J. Meyer, ${ }^{23, \dagger, q q}$ T. Miao,${ }^{75, *}$ F. Miconi,${ }^{19, \dagger}$ D. Mietlicki, ${ }^{93, *}$ A. Mitra, ${ }^{6, *}$ H. Miyake, ${ }^{41, *}$ S. Moed, ${ }^{75, *}$ N. Moggi, ${ }^{32 a, *}$ N. K. Mondal, ${ }^{30,}$ C. S. Moon, ${ }^{75, *, w}$ R. Moore, ${ }^{75, * \text { gg }}$ M. J. Morello, ${ }^{35 d, 35 a, *}$ A. Mukherjee, ${ }^{75, *}$ M. Mulhearn,${ }^{120, \dagger}$ Th. Muller, ${ }^{24, *}$ P. Murat, ${ }^{75, *}$

M. Mussini, ${ }^{32 \mathrm{~b}, 32 \mathrm{a}, *}$ J. Nachtman, ${ }^{75, *, s}$ Y. Nagai ${ }^{41, *}$ J. Naganoma ${ }^{40, *}$ E. Nagy, ${ }^{15,{ }^{\dagger}}$ I. Nakano, ${ }^{38, *}$ A. Napier, ${ }^{92, *}$

M. Narain, ${ }^{114, \dagger}$ R. Nayyar, ${ }^{66, \dagger}$ H. A. Neal, ${ }^{93, \dagger}$ J. P. Negret, ${ }^{7, \dagger}$ J. Nett, ${ }^{116, *}$ C. Neu, ${ }^{10, *}$ P. Neustroev, ${ }^{51, \dagger}$ H. T. Nguyen, ${ }^{120, \dagger}$

T. Nigmanov, ${ }^{113, *}$ L. Nodulman, ${ }^{74, *}$ S. Y. Noh, ${ }^{42, *}$ O. Norniella, ${ }^{80, *}$ T. Nunnemann, ${ }^{26, \dagger}$ L. Oakes, ${ }^{65, *}$ S. H. Oh, ${ }^{106, *}$ Y.D. Oh, ${ }^{42, *}$ I. Oksuzian, ${ }^{120, *}$ T. Okusawa, ${ }^{39, *}$ R. Orava, ${ }^{12, *}$ J. Orduna,${ }^{118, \dagger}$ L. Ortolan,${ }^{53, *}$ N. Osman, ${ }^{15, \dagger}$ J. Osta, ${ }^{83, *}$ C. Pagliarone, ${ }^{37 a, *}$ A. Pal,${ }^{115, \dagger}$ E. Palencia,${ }^{56, *, f}$ P. Palni, ${ }^{100, *}$ V. Papadimitriou, ${ }^{75, *}$ N. Parashar, ${ }^{82, \dagger}$ V. Parihar, ${ }^{114, \dagger}$ S. K. Park, ${ }^{43, \dagger}$ W. Parker, ${ }^{122, *}$ R. Partridge, ${ }^{114, \dagger, 00}$ N. Parua,${ }^{81, \dagger}$ A. Patwa, ${ }^{105, \dagger, v}$ G. Pauletta, ${ }^{37 \mathrm{c}, 37 \mathrm{a}, *}$ M. Paulini, ${ }^{112, *}$ C. Paus, ${ }^{91, *}$ B. Penning,${ }^{75, \dagger}$ M. Perfilov,${ }^{49, \dagger}$ Y. Peters,${ }^{23, \dagger}$ K. Petridis,${ }^{64, \dagger}$ G. Petrillo, ${ }^{103, \dagger}$ P. Pétroff, ${ }^{16, \dagger}$ T. J. Phillips, ${ }^{106, *}$ G. Piacentino, ${ }^{35 a, *}$ E. Pianori, ${ }^{111, *}$ J. Pilot, ${ }^{107, *}$ K. Pitts, ${ }^{80, *}$ C. Plager,${ }^{69, *}$ M.-A. Pleier, ${ }^{105, \dagger}$ V. M. Podstavkov,${ }^{7, \dagger}$

L. Pondrom, ${ }^{12, *}$ A. V. Popov ${ }^{50, \dagger}$ S. Poprocki,${ }^{75, *, \mathrm{k}}$ K. Potamianos, ${ }^{67, *}$ A. Pranko, ${ }^{67, *}$ M. Prewitt, ${ }^{118, \dagger}$ D. Price,${ }^{81, \dagger}$ N. Prokopenko, ${ }^{50, \dagger}$ F. Prokoshin, ${ }^{47, *, t}$ F. Ptohos, ${ }^{33, *, \mathrm{u}}$ G. Punzi, ${ }^{35 \mathrm{~b}, 35 \mathrm{a}, *}$ J. Qian, ${ }^{93, \dagger}$ A. Quadt, ${ }^{23, \dagger}$ B. Quinn, ${ }^{96, \dagger}$ N. Ranjan, ${ }^{84, *}$

P. N. Ratoff ${ }^{60, \dagger}$ I. Razumov, ${ }^{50, \dagger}$ I. Redondo Fernández, ${ }^{55, *}$ P. Renton, ${ }^{65, *}$ M. Rescigno, ${ }^{36 a, *}$ F. Rimondi, ${ }^{32 a, *, a}$

I. Ripp-Baudot, ${ }^{19, \dagger}$ L. Ristori, ${ }^{35,75, *}$ F. Rizatdinova, ${ }^{110, \dagger}$ A. Robson, ${ }^{59, *}$ T. Rodriguez,,${ }^{11, *}$ S. Rolli, ${ }^{92, *, v}$ M. Rominsky, ${ }^{75, \dagger}$ M. Ronzani, ${ }^{35 b, 35 a, *}$ R. Roser, ${ }^{75, *}$ J. L. Rosner, ${ }^{76, *}$ A. Ross,${ }^{60, \dagger}$ C. Royon, ${ }^{18, \dagger}$ P. Rubinov, ${ }^{75, \dagger}$ R. Ruchti, ${ }^{83, \dagger}$ F. Ruffini, ${ }^{35 c, 35 a, *}$

A. Ruiz, ${ }^{56, *}$ J. Russ, ${ }^{12, *}$ V. Rusu, ${ }^{75}, *$ G. Sajot,${ }^{14, \dagger}$ W. K. Sakumoto, ${ }^{103, *}$ Y. Sakurai ${ }^{40, *}$ A. Sánchez-Hernández, ${ }^{44, \dagger}$ M.P. Sanders, ${ }^{26, \dagger}$ L. Santi, ${ }^{37 \mathrm{c}, 37 \mathrm{a}, *}$ A. S. Santos, ${ }^{1, \dagger, \mathrm{pp}}$ K. Sato, ${ }^{41, *}$ G. Savage,${ }^{75, \dagger}$ V. Saveliev, ${ }^{75, *, \mathrm{~h}}$ A. Savoy-Navarro, ${ }^{75, *, w}$ L. Sawyer, ${ }^{87, \dagger}$ T. Scanlon, ${ }^{62, \dagger}$ R. D. Schamberger, ${ }^{104, \dagger}$ Y. Scheglov, ${ }^{51, \dagger}$ H. Schellman,${ }^{79, \dagger}$ P. Schlabach,${ }^{75, *}$ E. E. Schmidt, ${ }^{75, *}$ C. Schwanenberger, ${ }^{64, \dagger}$ T. Schwarz, ${ }^{93, *}$ R. Schwienhorst ${ }^{95, \dagger}$ L. Scodellaro, ${ }^{56, *}$ F. Scuri, ${ }^{35 a, *}$ S. Seidel ${ }^{100, *}$ Y. Seiya, ${ }^{39, *}$ J. Sekaric, ${ }^{86, \dagger}$ A. Semenov, ${ }^{47, *}$ H. Severini, ${ }^{109, \dagger}$ F. Sforza, ${ }^{35 b, 35 a, *}$ E. Shabalina, ${ }^{23, \dagger}$ S. Z. Shalhout, ${ }^{68, *}$ V. Shary, ${ }^{18, \dagger}$ S. Shaw,${ }^{95,}$ A. A. Shchukin, ${ }^{50, \dagger}$ T. Shears, ${ }^{61, *}$ P. F. Shepard, ${ }^{113, *}$ M. Shimojima, ${ }^{41, *, x}$ M. Shochet,${ }^{76, *}$

I. Shreyber-Tecker, ${ }^{48, *}$ V. Simak, ${ }^{9, \dagger}$ A. Simonenko, ${ }^{47, *}$ P. Sinervo, ${ }^{4, *}$ P. Skubic,${ }^{109, \dagger}$ P. Slattery, ${ }^{103, \dagger}$ K. Sliwa, ${ }^{92, *}$

D. Smirnov, ${ }^{83, \dagger}$ J. R. Smith, ${ }^{68, *}$ F. D. Snider, ${ }^{75, *}$ G. R. Snow,${ }^{97,}$ J. Snow, ${ }^{108, \dagger}$ S. Snyder, ${ }^{105, \dagger}$ S. Söldner-Rembold ${ }^{64, \dagger}$

H. Song,${ }^{113, *}$ L. Sonnenschein, ${ }^{21, \dagger}$ V. Sorin,${ }^{53, *}$ K. Soustruznik, ${ }^{8, \dagger}$ M. Stancari, ${ }^{75, *}$ R. St. Denis,${ }^{59, *}$ J. Stark, ${ }^{14, \dagger}$

B. Stelzer, ${ }^{4, *}$ O. Stelzer-Chilton, ${ }^{4, *}$ D. Stentz, ${ }^{75, *, c}$ D. A. Stoyanova, ${ }^{50, \dagger}$ M. Strauss, ${ }^{109, \dagger}$ J. Strologas, ${ }^{100, *}$ Y. Sudo, ${ }^{41, *}$ A. Sukhanov ${ }^{75, *}$ I. Suslov,${ }^{47, *}$ L. Suter,${ }^{64, \dagger}$ P. Svoisky, ${ }^{109, \dagger}$ K. Takemasa, ${ }^{41, *}$ Y. Takeuchi, ${ }^{41, *}$ J. Tang,${ }^{76, *}$ M. Tecchio, ${ }^{93, *}$ P. K. Teng, ${ }^{6, *}$ J. Thom,${ }^{75, *, k}$ E. Thomson, ${ }^{111, *}$ V. Thukral, ${ }^{116, *}$ M. Titov, ${ }^{18, \dagger}$ D. Toback, ${ }^{116, *}$ S. Tokar, ${ }^{52, *}$ V. V. Tokmenin, ${ }^{47, \dagger}$

K. Tollefson, ${ }^{95, *}$ T. Tomura, ${ }^{41, *}$ D. Tonelli,${ }^{75, *, f}$ S. Torre,${ }^{33, *}$ D. Torretta,${ }^{75, *}$ P. Totaro, ${ }^{34 a, *}$ M. Trovato,${ }^{35 d, 35 a, *}$

Y.-T. Tsai, ${ }^{103, \dagger}$ D. Tsybychev ${ }^{104, \dagger}$ B. Tuchming, ${ }^{18, \dagger}$ C. Tully, ${ }^{98, \dagger}$ F. Ukegawa, ${ }^{41, *}$ S. Uozumi, ${ }^{42, *}$ L. Uvarov, ${ }^{51, \dagger}$

S. Uvarov,${ }^{51, \dagger}$ S. Uzunyan, ${ }^{78, \dagger}$ R. Van Kooten,${ }^{81, \dagger}$ W. M. van Leeuwen, ${ }^{45, \dagger}$ N. Varelas, ${ }^{77, \dagger}$ E. W. Varnes,${ }^{66, \dagger}$ I. A. Vasilyev, ${ }^{50, \dagger}$

F. Vázquez, ${ }^{72, *, d}$ G. Velev, ${ }^{75, *}$ C. Vellidis ${ }^{75, *}$ A. Y. Verkheev, ${ }^{47, \dagger}$ C. Vernieri, ${ }^{35 d, 35 a, *}$ L. S. Vertogradov, ${ }^{47, \dagger}$

M. Verzocchi, ${ }^{75, \dagger}$ M. Vesterinen ${ }^{64, \dagger}$ M. Vidal, ${ }^{84, *}$ D. Vilanova, ${ }^{18, \dagger}$ R. Vilar, ${ }^{56, *}$ J. Vizán, ${ }^{56, *, \text { hh }}$ M. Vogel, ${ }^{100, *}$ P. Vokac, ${ }^{9, \dagger}$ G. Volpi ${ }^{33, *}$ P. Wagner, ${ }^{111, *}$ H.D. Wahl, ${ }^{73, \dagger}$ R. Wallny, ${ }^{69, *}$ S. M. Wang, ${ }^{6, *}$ M. H. L. S. Wang,${ }^{75, \dagger}$ R.-J. Wang, ${ }^{89, \dagger}$ A. Warburton, ${ }^{4, *} \mathrm{~J}$. Warchol, ${ }^{83, \dagger}$ D. Waters ${ }^{63, *}$ G. Watts, ${ }^{121, \dagger}$ M. Wayne, ${ }^{83, \dagger}$ J. Weichert, ${ }^{25, \dagger}$ L. Welty-Rieger, ${ }^{79, \dagger}$ 
W. C. Wester III, ${ }^{75, *}$ D. Whiteson, ${ }^{111, *, y}$ A. B. Wicklund, ${ }^{74, *}$ S. Wilbur, ${ }^{76, *}$ H. H. Williams, ${ }^{111, *}$ M. R. J. Williams ${ }^{81, \dagger}$ G. W. Wilson, ${ }^{86, \dagger}$ J. S. Wilson, ${ }^{93, *}$ P. Wilson, ${ }^{75, *}$ B. L. Winer, ${ }^{107, *}$ P. Wittich, ${ }^{75, *, k}$ M. Wobisch, ${ }^{87, \dagger}$ S. Wolbers, ${ }^{75, *}$ H. Wolfe, ${ }^{107, *}$ D. R. Wood,${ }^{89, \dagger}$ T. Wright,${ }^{93, *}$ X. Wu,${ }^{58, *}$ Z. Wu,${ }^{19, *}$ T. R. Wyatt, ${ }^{64, \dagger}$ Y. Xie, ${ }^{75, \dagger}$ R. Yamada,${ }^{75, \dagger}$ K. Yamamoto, ${ }^{39, *}$ D. Yamato, ${ }^{39, *}$ S. Yang, ${ }^{5, \dagger}$ T. Yang,${ }^{75, *}$ U. K. Yang, ${ }^{76, *, z}$ Y. C. Yang, ${ }^{42, *}$ W.-M. Yao,${ }^{67, *}$ T. Yasuda, ${ }^{75, \dagger}$ Y. A. Yatsunenko, ${ }^{47, \dagger}$ W. Ye,${ }^{104, \dagger}$ Z. Ye,${ }^{75, \dagger}$ G.P. Yeh,${ }^{75, *}$ K. Yi, ${ }^{75, *, s}$ H. Yin,${ }^{75, \dagger}$ K. Yip,${ }^{105, \dagger}$ J. Yoh ${ }^{75, *}$ K. Yorita, ${ }^{40, *}$ T. Yoshida, ${ }^{39, * \text { aa }}$ S. W. Youn, ${ }^{75, \dagger}$ G. B. Yu,${ }^{106, *}$ I. Yu, ${ }^{42, *}$ J. M. Yu,${ }^{93, \dagger}$ A. Zanetti ${ }^{37 a, *}$ Y. Zeng, ${ }^{106, *}$ J. Zennamo, ${ }^{101, \dagger}$ T. G. Zhao, ${ }^{64, \dagger}$ B. Zhou, ${ }^{93, \dagger}$ C. Zhou, ${ }^{106, *}$ J. Zhu,${ }^{93, \dagger}$ M. Zielinski, ${ }^{103, \dagger}$ D. Zieminska, ${ }^{81, \dagger}$ L. Zivkovic, ${ }^{17, \dagger}$ and S. Zucchelli ${ }^{32 b, 32 a, *}$

\section{$\left({ }^{*}\right.$ CDF Collaboration) ('D0 Collaboration)}

${ }^{1}$ LAFEX, Centro Brasileiro de Pesquisas Físicas, Rio de Janeiro, Brazil

${ }^{2}$ Universidade do Estado do Rio de Janeiro, Rio de Janeiro, Brazil

${ }^{3}$ Universidade Federal do ABC, Santo André, Brazil

${ }^{4}$ Institute of Particle Physics: McGill University, Montréal, Québec, Canada H3A 2T8; Simon Fraser University, Burnaby, British Columbia, Canada V5A 1S6; University of Toronto, Toronto, Ontario, Canada M5S 1A7; and TRIUMF, Vancouver, British Columbia, V6T 2A3, Canada

${ }^{5}$ University of Science and Technology of China, Hefei, People's Republic of China

${ }^{6}$ Institute of Physics, Academia Sinica, Taipei, Taiwan 11529, Republic of China

${ }^{7}$ Universidad de los Andes, Bogotá, Colombia

${ }^{8}$ Charles University, Faculty of Mathematics and Physics, Center for Particle Physics, Prague, Czech Republic

${ }^{9}$ Czech Technical University in Prague, Prague, Czech Republic

${ }^{10}$ Center for Particle Physics, Institute of Physics, Academy of Sciences of the Czech Republic, Prague, Czech Republic

${ }^{11}$ Universidad San Francisco de Quito, Quito, Ecuador

${ }^{12}$ Division of High Energy Physics, Department of Physics, University of Helsinki and Helsinki Institute of Physics, FIN-00014, Helsinki, Finland

${ }^{13}$ LPC, Université Blaise Pascal, CNRS/IN2P3, Clermont, France

${ }^{14}$ LPSC, Université Joseph Fourier Grenoble 1, CNRS/IN2P3, Institut National Polytechnique de Grenoble, Grenoble, France

${ }^{15}$ CPPM, Aix-Marseille Université, CNRS/IN2P3, Marseille, France

${ }^{16}$ LAL, Université Paris-Sud, CNRS/IN2P3, Orsay, France

${ }^{17}$ LPNHE, Universités Paris VI and VII, CNRS/IN2P3, Paris, France

${ }^{18}$ CEA, Irfu, SPP, Saclay, France

${ }^{19}$ IPHC, Université de Strasbourg, CNRS/IN2P3, Strasbourg, France

${ }^{20} I P N L$, Université Lyon 1, CNRS/IN2P3, Villeurbanne, France and Université de Lyon, Lyon, France

${ }^{21}$ III. Physikalisches Institut A, RWTH Aachen University, Aachen, Germany

${ }^{22}$ Physikalisches Institut, Universität Freiburg, Freiburg, Germany

${ }^{23}$ II. Physikalisches Institut, Georg-August-Universität Göttingen, Göttingen, Germany

${ }^{24}$ Institut für Experimentelle Kernphysik, Karlsruhe Institute of Technology, D-76131 Karlsruhe, Germany

${ }^{25}$ Institut für Physik, Universität Mainz, Mainz, Germany

${ }^{26}$ Ludwig-Maximilians-Universität München, München, Germany

${ }^{27}$ University of Athens, 15771 Athens, Greece

${ }^{28}$ Panjab University, Chandigarh, India

${ }^{29}$ Delhi University, Delhi, India

${ }^{30}$ Tata Institute of Fundamental Research, Mumbai, India

${ }^{31}$ University College Dublin, Dublin, Ireland

${ }^{32 \mathrm{a}}$ Istituto Nazionale di Fisica Nucleare Bologna, I-40127 Bologna, Italy

${ }^{32 \mathrm{~b}}$ University of Bologna, I-40127 Bologna, Italy

${ }^{33}$ Laboratori Nazionali di Frascati, Istituto Nazionale di Fisica Nucleare, I-00044 Frascati, Italy

${ }^{34 \mathrm{a}}$ Istituto Nazionale di Fisica Nucleare, Sezione di Padova-Trento, I-35131 Padova, Italy

${ }^{34 \mathrm{~b}}$ University of Padova, I-35131 Padova, Italy

${ }^{35 a}$ Istituto Nazionale di Fisica Nucleare Pisa, I-56127 Pisa, Italy

${ }^{35 \mathrm{~b}}$ University of Pisa, I-56127 Pisa, Italy

${ }^{35 c}$ University of Siena, I-56127 Pisa, Italy

${ }^{35 \mathrm{~d}}$ Scuola Normale Superiore, I-56127 Pisa, Italy

${ }^{35 \mathrm{e}}$ INFN Pavia and University of Pavia, I-27100 Pavia, Italy

${ }^{36 a}$ Istituto Nazionale di Fisica Nucleare, Sezione di Roma 1, I-00185 Roma, Italy

${ }^{36 \mathrm{~b}}$ Sapienza Università di Roma, I-00185 Roma, Italy

${ }^{37}$ Istituto Nazionale di Fisica Nucleare Trieste/Udine, I-34127 Trieste, Italy 
${ }^{37 \mathrm{~b}}$ University of Trieste, I-34127 Trieste, Italy

${ }^{37 \mathrm{c}}$ University of Udine, I-33100 Udine, Italy

${ }^{38}$ Okayama University, Okayama 700-8530, Japan

${ }^{39}$ Osaka City University, Osaka 588, Japan

${ }^{40}$ Waseda University, Tokyo 169, Japan

${ }^{41}$ University of Tsukuba, Tsukuba, Ibaraki 305, Japan

${ }^{42}$ Center for High Energy Physics: Kyungpook National University, Daegu 702-701, Korea;

Seoul National University, Seoul 151-742, Korea; Sungkyunkwan University, Suwon 440-746, Korea;

Korea Institute of Science and Technology Information, Daejeon 305-806, Korea;

Chonnam National University, Gwangju 500-757, Korea; Chonbuk National University, Jeonju 561-756, Korea;

Ewha Womans University, Seoul, 120-750, Korea

${ }^{43}$ Korea Detector Laboratory, Korea University, Seoul, Korea

${ }^{44}$ CINVESTAV, Mexico City, Mexico

${ }^{45}$ Nikhef, Science Park, Amsterdam, Netherlands

${ }^{46}$ Radboud University Nijmegen, Nijmegen, Netherlands

${ }^{47}$ Joint Institute for Nuclear Research, Dubna, Russia

${ }^{48}$ Institution for Theoretical and Experimental Physics, ITEP, Moscow 117259, Russia

${ }^{49}$ Moscow State University, Moscow, Russia

${ }^{50}$ Institute for High Energy Physics, Protvino, Russia

${ }^{51}$ Petersburg Nuclear Physics Institute, St. Petersburg, Russia

${ }^{52}$ Comenius University, 84248 Bratislava, Slovakia; Institute of Experimental Physics, 04001 Kosice, Slovakia

${ }^{53}$ Institut de Fisica d'Altes Energies, ICREA, Universitat Autonoma de Barcelona, E-08193, Bellaterra (Barcelona), Spain

${ }^{54}$ Institució Catalana de Recerca i Estudis Avançats (ICREA) and Institut de Física d'Altes Energies (IFAE), Barcelona, Spain

${ }^{55}$ Centro de Investigaciones Energeticas Medioambientales y Tecnologicas, E-28040 Madrid, Spain

${ }^{56}$ Instituto de Fisica de Cantabria, CSIC-University of Cantabria, 39005 Santander, Spain

${ }^{57}$ Uppsala University, Uppsala, Sweden

${ }^{58}$ University of Geneva, CH-1211 Geneva 4, Switzerland

${ }^{59}$ Glasgow University, Glasgow G12 8QQ, United Kingdom

${ }^{60}$ Lancaster University, Lancaster LA1 4YB, United Kingdom

${ }^{61}$ University of Liverpool, Liverpool L69 7ZE, United Kingdom

${ }^{62}$ Imperial College London, London SW7 2AZ, United Kingdom

${ }^{63}$ University College London, London WC1E 6BT, United Kingdom

${ }^{64}$ The University of Manchester, Manchester M13 9PL, United Kingdom

${ }^{65}$ University of Oxford, Oxford OX1 3RH, United Kingdom

${ }^{66}$ University of Arizona, Tucson, Arizona 85721, USA

${ }^{67}$ Ernest Orlando Lawrence Berkeley National Laboratory, Berkeley, California 94720, USA

${ }^{68}$ University of California, Davis, Davis, California 95616, USA

${ }^{69}$ University of California, Los Angeles, Los Angeles, California 90024, USA

${ }^{70}$ University of California, Riverside, Riverside, California 92521, USA

${ }^{71}$ Yale University, New Haven, Connecticut 06520, USA

${ }^{72}$ University of Florida, Gainesville, Florida 32611, USA

${ }^{73}$ Florida State University, Tallahassee, Florida 32306, USA

${ }^{74}$ Argonne National Laboratory, Argonne, Illinois 60439, USA

${ }^{75}$ Fermi National Accelerator Laboratory, Batavia, Illinois 60510, USA

${ }^{76}$ Enrico Fermi Institute, University of Chicago, Chicago, Illinois 60637, USA

${ }^{77}$ University of Illinois at Chicago, Chicago, Illinois 60607, USA

${ }^{78}$ Northern Illinois University, DeKalb, Illinois 60115, USA

${ }^{79}$ Northwestern University, Evanston, Illinois 60208, USA

${ }^{80}$ University of Illinois, Urbana, Illinois 61801, USA

${ }^{81}$ Indiana University, Bloomington, Indiana 47405, USA

${ }^{82}$ Purdue University Calumet, Hammond, Indiana 46323, USA

${ }^{83}$ University of Notre Dame, Notre Dame, Indiana 46556, USA

${ }^{84}$ Purdue University, West Lafayette, Indiana 47907, USA

${ }^{85}$ Iowa State University, Ames, Iowa 50011, USA

${ }^{86}$ University of Kansas, Lawrence, Kansas 66045, USA

${ }^{87}$ Louisiana Tech University, Ruston, Louisiana 71272, USA

${ }^{88}$ The Johns Hopkins University, Baltimore, Maryland 21218, USA

${ }^{89}$ Northeastern University, Boston, Massachusetts 02115, USA

${ }^{90}$ Harvard University, Cambridge, Massachusetts 02138, USA 
${ }^{91}$ Massachusetts Institute of Technology, Cambridge, Massachusetts 02139, USA

${ }^{92}$ Tufts University, Medford, Massachusetts 02155, USA

${ }^{93}$ University of Michigan, Ann Arbor, Michigan 48109, USA

${ }^{94}$ Wayne State University, Detroit, Michigan 48201, USA

${ }^{95}$ Michigan State University, East Lansing, Michigan 48824, USA

${ }^{96}$ University of Mississippi, University, Mississippi 38677, USA

${ }^{97}$ University of Nebraska, Lincoln, Nebraska 68588, USA

${ }^{98}$ Princeton University, Princeton, New Jersey 08544, USA

${ }^{99}$ Rutgers University, Piscataway, New Jersey 08855, USA

${ }^{100}$ University of New Mexico, Albuquerque, New Mexico 87131, USA

${ }^{101}$ State University of New York, Buffalo, New York 14260, USA

${ }^{102}$ The Rockefeller University, New York, New York 10065, USA

${ }^{103}$ University of Rochester, Rochester, New York 14627, USA

${ }^{104}$ State University of New York, Stony Brook, New York 11794, USA

${ }^{105}$ Brookhaven National Laboratory, Upton, New York 11973, USA

${ }^{106}$ Duke University, Durham, North Carolina 27708, USA

${ }^{107}$ The Ohio State University, Columbus, Ohio 43210, USA

${ }^{108}$ Langston University, Langston, Oklahoma 73050, USA

${ }^{109}$ University of Oklahoma, Norman, Oklahoma 73019, USA

${ }^{110}$ Oklahoma State University, Stillwater, Oklahoma 74078, USA

${ }^{111}$ University of Pennsylvania, Philadelphia, Pennsylvania 19104, USA

${ }^{112}$ Carnegie Mellon University, Pittsburgh, Pennsylvania 15213, USA

${ }^{113}$ University of Pittsburgh, Pittsburgh, Pennsylvania 15260, USA

${ }^{114}$ Brown University, Providence, Rhode Island 02912, USA

${ }^{115}$ University of Texas, Arlington, Texas 76019, USA

${ }^{116}$ Mitchell Institute for Fundamental Physics and Astronomy, Texas A\&M University, College Station, Texas 77843, USA

${ }^{117}$ Southern Methodist University, Dallas, Texas 75275, USA

${ }^{118}$ Rice University, Houston, Texas 77005, USA

${ }^{119}$ Baylor University, Waco, Texas 76798, USA

${ }^{120}$ University of Virginia, Charlottesville, Virginia 22904, USA

${ }^{121}$ University of Washington, Seattle, Washington 98195, USA

${ }^{122}$ University of Wisconsin, Madison, Wisconsin 53706, USA

(Received 26 March 2013; published 17 September 2013)

We combine searches by the CDF and D0 Collaborations for the standard model Higgs boson with mass in the range $90-200 \mathrm{GeV} / c^{2}$ produced in the gluon-gluon fusion, $W H, Z H, t \bar{t} H$, and vector boson fusion processes, and decaying in the $H \rightarrow b \bar{b}, H \rightarrow W^{+} W^{-}, H \rightarrow Z Z, H \rightarrow \tau^{+} \tau^{-}$, and $H \rightarrow \gamma \gamma$ modes. The

${ }^{\mathrm{a}}$ Deceased.

${ }^{\mathrm{b}}$ Visitor from Universidad de Oviedo, E-33007 Oviedo, Spain.

${ }^{c}$ Visitor from Northwestern University, Evanston, IL 60208, USA.

${ }^{\mathrm{d}}$ Visitor from Universidad Iberoamericana, Mexico D.F., Mexico.

${ }^{\mathrm{e}}$ Visitor from ETH, 8092 Zürich, Switzerland.

${ }^{\mathrm{f}}$ Visitor from CERN, CH-1211 Geneva, Switzerland.

${ }^{\mathrm{g}}$ Visitor from Queen Mary, University of London, London, E1 4NS, United Kingdom.

${ }^{\mathrm{h}}$ Visitor from National Research Nuclear University, Moscow, Russia.

${ }^{\mathrm{i}}$ Visitor from Yarmouk University, Irbid 211-63, Jordan.

${ }^{\mathrm{j}}$ Visitor from Muons, Inc., Batavia, IL 60510, USA.

${ }^{\mathrm{k}}$ Visitor from Cornell University, Ithaca, NY 14853, USA.

${ }^{1}$ Visitor from Kansas State University, Manhattan, KS 66506, USA.

${ }^{\mathrm{m}}$ Visitor from Kinki University, Higashi-Osaka City, Japan 577-8502.

${ }^{\mathrm{n}}$ Visitor from University of Notre Dame, Notre Dame, IN 46556, USA.

${ }^{\circ}$ Visitor from University of Melbourne, Victoria 3010, Australia.

${ }^{\mathrm{p}}$ Visitor from Institute of Physics, Academy of Sciences of the Czech Republic, Czech Republic.

${ }^{\mathrm{q}}$ Visitor from Istituto Nazionale di Fisica Nucleare, Sezione di Cagliari, 09042 Monserrato (Cagliari), Italy.

${ }^{\mathrm{r}}$ Visitor from University College Dublin, Dublin 4, Ireland.

${ }^{\mathrm{s}}$ Visitor from University of Iowa, Iowa City, IA 52242, USA.

${ }^{\mathrm{t}}$ Visitor from Universidad Tecnica Federico Santa Maria, 110v Valparaiso, Chile.

${ }^{\mathrm{u}}$ Visitor from University of Cyprus, Nicosia CY-1678, Cyprus.

${ }^{\vee}$ Visitor from Office of Science, U.S. Department of Energy, Washington, DC 20585, USA.

${ }^{\text {w}}$ Visitor from CNRS-IN2P3, Paris, F-75205 France.

${ }^{\mathrm{x}}$ Visitor from Nagasaki Institute of Applied Science, Nagasaki, Japan. 
data correspond to integrated luminosities of up to $10 \mathrm{fb}^{-1}$ and were collected at the Fermilab Tevatron in $p \bar{p}$ collisions at $\sqrt{s}=1.96 \mathrm{TeV}$. The searches are also interpreted in the context of fermiophobic and fourth generation models. We observe a significant excess of events in the mass range between 115 and $140 \mathrm{GeV} / c^{2}$. The local significance corresponds to 3.0 standard deviations at $m_{H}=$ $125 \mathrm{GeV} / c^{2}$, consistent with the mass of the Higgs boson observed at the LHC, and we expect a local significance of 1.9 standard deviations. We separately combine searches for $H \rightarrow b \bar{b}, H \rightarrow W^{+} W^{-}$, $H \rightarrow \tau^{+} \tau^{-}$, and $H \rightarrow \gamma \gamma$. The observed signal strengths in all channels are consistent with the presence of a standard model Higgs boson with a mass of $125 \mathrm{GeV} / c^{2}$.

DOI: 10.1103/PhysRevD.88.052014

PACS numbers: 13.85.Rm, 14.80.Bn

\section{INTRODUCTION}

Within the standard model (SM) [1], spontaneous breaking of electroweak symmetry gives mass to the $W$ and $Z$ bosons [2], and to the fundamental fermions via their Yukawa interactions with the Higgs field. In the SM, the symmetry-breaking mechanism predicts the existence of one neutral scalar particle, the Higgs boson, whose mass $\left(m_{H}\right)$ is a free parameter.

Precision electroweak data, including the recently updated measurements of the $W$-boson and top-quark masses from the CDF and D0 Collaborations [3,4], yield an indirect constraint on the allowed mass of the Higgs boson, $m_{H}<152 \mathrm{GeV} / c^{2}$ [5], at the $95 \%$ confidence level (C.L.) [6]. Direct searches at LEP2 exclude SM Higgs boson masses below $114.4 \mathrm{GeV} / c^{2}$ [7]. The ATLAS and CMS Collaborations at the Large Hadron Collider (LHC) have recently reported the observation of a new boson with mass of around $125 \mathrm{GeV} / c^{2}[8,9]$. Much of the sensitivity of the LHC searches comes from gluon-gluon fusion $(g g \rightarrow H)$ production and Higgs boson decays to $\gamma \gamma, Z Z$, and $W^{+} W^{-}$. Published searches for associated production $V H \rightarrow V b \bar{b}$ at the LHC, where $V=W$ or $Z[10,11]$, have not yet reached sensitivity to SM Higgs boson production. The CDF and D0 Collaborations have recently reported combined evidence for a particle, with a mass consistent with that of the new boson observed at the LHC, produced in association with a $W$ or $Z$ boson and decaying to a bottom-antibottom quark pair [12].

In this article, we combine the most recent results of SM Higgs boson searches in $p \bar{p}$ collisions at $\sqrt{s}=1.96 \mathrm{TeV}$ using the full Tevatron Run II integrated luminosity of up to $10 \mathrm{fb}^{-1}$ per experiment. The analyses combined here seek signals of Higgs bosons in the mass range $90-200 \mathrm{GeV} / c^{2}$, produced in association with a vector boson $(q \bar{q} \rightarrow V H)$, in association with top quarks, through gluon-gluon fusion, and through vector boson fusion (VBF) $\left(q \bar{q} \rightarrow q^{\prime} \bar{q}^{\prime} H\right)$. The Higgs boson decay modes studied are $H \rightarrow b \bar{b}, H \rightarrow$ $W^{+} W^{-}, H \rightarrow Z Z, H \rightarrow \tau^{+} \tau^{-}$, and $H \rightarrow \gamma \gamma$. For Higgs boson masses greater than $130 \mathrm{GeV} / c^{2}$, searches for $H \rightarrow$ $W^{+} W^{-}$decays with subsequent leptonic $W$ decays provide the greatest sensitivity. Below $130 \mathrm{GeV} / c^{2}$, sensitivity comes mainly from associated $V H$ production, with the $H$ boson decaying to $b \bar{b}$ and the $W$ or $Z$ boson decaying leptonically. While we present our results in the full mass range, we also focus specifically on the mass hypothesis $m_{H}=125 \mathrm{GeV} / c^{2}$, due to the recent LHC findings. Specifically, we show the sensitivity of the searches over the full mass range to a SM Higgs boson signal with $m_{H}=125 \mathrm{GeV} / c^{2}$. Previous Tevatron SM combinations, focused respectively on the $H \rightarrow b \bar{b}$ and $H \rightarrow W^{+} W^{-}$ decay modes, are published in Refs. [12,13]. The results

\footnotetext{
${ }^{\mathrm{y}}$ Visitor from University of California Irvine, Irvine, CA 92697, USA.

${ }^{\mathrm{z}}$ Visitor from University of Manchester, Manchester M13 9PL, United Kingdom.

${ }^{\text {aa }}$ Visitor from University of Fukui, Fukui City, Fukui Prefecture, Japan 910-0017.

${ }^{\mathrm{bb}}$ Visitor from University of British Columbia, Vancouver, BC V6T 1Z1, Canada.

${ }^{\mathrm{cc}}$ Visitor from University of Zürich, 8006 Zürich, Switzerland.

${ }^{\mathrm{dd}}$ Visitor from Hampton University, Hampton, VA 23668, USA.

${ }^{\text {ee } V i s i t o r ~ f r o m ~ B r o o k h a v e n ~ N a t i o n a l ~ L a b o r a t o r y, ~ U p t o n, ~ N Y ~ 11973, ~ U S A . ~}$

${ }^{\mathrm{ff}}$ Visitor from Los Alamos National Laboratory, Los Alamos, NM 87544, USA.

${ }^{g g}$ Visitor from Massachusetts General Hospital and Harvard Medical School, Boston, MA 02114, USA.

${ }^{\text {hh }}$ Visitor from Université catholique de Louvain, 1348 Louvain-La-Neuve, Belgium.

${ }^{i i}$ Visitor from Augustana College, Sioux Falls, SD, USA.

${ }^{\mathrm{jj}}$ Visitor from The University of Liverpool, Liverpool, UK.

${ }^{\mathrm{k}}$ Visitor from Universidad Michoacana de San Nicolas de Hidalgo, Morelia, Mexico.

${ }^{11}$ Visitor from DESY, Hamburg, Germany.

${ }^{\mathrm{mm}}$ Visitor from University College London, London, UK.

${ }^{\mathrm{nn}}$ Visitor from Centro de Investigacion en Computacion - IPN, Mexico City, Mexico.

${ }^{\mathrm{oo}}$ Visitor from SLAC, Menlo Park, CA, USA.

${ }^{p p}$ Visitor from Universidade Estadual Paulista, São Paulo, Brazil.

${ }^{\mathrm{qq}}$ Visitor from Karlsruher Institut für Technologie (KIT) - Steinbuch Centre for Computing (SCC).
} 
presented here are based on the combinations of the searches from each experiment as published in Refs. [14,15].

This article is structured as follows. Section II discusses the simulation methods used to predict the yields from the signal and SM background processes. Section III briefly describes the CDF and D0 detectors. Section IV describes the event selections used by the various analyses and Section V presents the data. Section VI provides a brief introduction to the statistical procedures used and Section VII discusses the different sources of systematic uncertainties and how they are controlled. Sections VIII and IX present the results in the contexts of the SM and extensions to it. Section X summarizes the article.

\section{EVENT SIMULATION}

Higgs boson signal events are simulated using the leading-order (LO) calculation from PYTHIA [16], with CTEQ5L (CDF) and CTEQ6L1 (D0) [17] parton distribution functions (PDFs). The normalization of these Monte Carlo (MC) samples is obtained using the highestorder cross-section calculation available for the corresponding production process. The cross section for the gluon-gluon fusion process is calculated at next-to-nextto-leading order (NNLO) in quantum chromodynamics (QCD) with soft gluon resummation to next-to-next-toleading-log (NNLL) accuracy $[18,19]$. These calculations include two-loop electroweak corrections, and also threeloop $\mathcal{O}\left(\alpha \alpha_{s}\right)$ corrections. The $W H$ and $Z H$ cross-section calculations are performed at NNLO precision in QCD and next-to-leading-order (NLO) precision in the electroweak corrections [20]. The VBF cross section is computed at NNLO in QCD [21], and the electroweak corrections are computed with the HAWK program [22]. The $t \bar{t} H$ production cross sections are taken from Ref. [23]. The signal production cross sections are computed using the MSTW2008 PDF set [24], except for the $t \bar{t} H$ production cross section which uses the CTEQ6M [17] PDF set. The Higgs boson decay branching fractions are from Ref. [25] and rely on calculations using HDECAY [26] and PROPHECY4F [27]. The distribution of the transverse momentum $\left(p_{T}\right)$ of the Higgs boson in the PYTHIA-generated gluon-fusion sample is reweighted to match the $p_{T}$ as calculated by HQT [28], at NNLL and NNLO accuracy.

We model SM and instrumental background processes using a mixture of $\mathrm{MC}$ and data-driven methods. In the $\mathrm{CDF}$ analyses, backgrounds from SM processes with electroweak gauge bosons or top quarks are modeled using PYTHIA, ALPGEN [29], MC@NLO [30], and HERWIG [31]. For D0, these backgrounds are modeled using PYTHIA, ALPGEN, and SINGLETOP [32]. An interface to PYTHIA provides parton showering and hadronization for generators without this functionality.

Diboson $(W W, W Z, Z Z)$ MC samples are normalized using the NLO calculations from MCFM [33]. For top-quark-pair production $(t \bar{t})$, we use a production cross section of $7.04 \pm 0.49 \mathrm{pb}$ [34], which is based on a topquark mass of $173 \mathrm{GeV} / c^{2}$ [4] and MSTW 2008 PDFs [24]. The single-top-quark production cross section is taken to be $3.15 \pm 0.31 \mathrm{pb}$ [35]. For many analyses, the $V+$ jet processes are normalized using the NNLO crosssection calculations of Ref. [36], though in some cases data-driven techniques are used. Likewise, the normalizations of the instrumental, multijet and, for the CDF searches, the $V+$ heavy-flavor jet backgrounds [37] are constrained from data samples where the expected signalto-background ratio is several orders of magnitude smaller than in the search samples. For the D0 searches, the $V+$ light-flavor is normalized to data in a control region, and the $V+$ heavy-flavor normalization, relative to the $V+$ light-flavor, is taken from MCFM. In addition, for the D0 searches, prior to $b$-tagging [38] $V+$ jets samples are compared to data and corrections applied to mitigate any discrepancies in kinematic distributions.

All MC samples are processed through a GEANT [39] simulation of the detector and reconstructed in the same way as data. The effects of instrumental noise and additional $p \bar{p}$ interactions are modeled using MC in the CDF analyses, while recorded data from randomly selected beam crossings with the same instantaneous luminosity profile as data are overlaid onto the MC events in the D0 analyses. In the entire Run II data sample, the average number of reconstructed primary vertices is approximately 3 -including the hard scatter.

For the $H \rightarrow W^{+} W^{-}$analyses, the dominant irreducible background process is diboson production, while the dominant reducible backgrounds are $Z / \gamma^{*}+$ jets, $t \bar{t}, W+\gamma$, $W+$ jets, and multijet production where in the latter three cases photons or jets can be misidentified as leptons. For the analyses targeting $H \rightarrow b \bar{b}$ the main backgrounds originate from $V+$ heavy-flavor-jets and $t \bar{t}$ production.

\section{DETECTORS AND OBJECT RECONSTRUCTION}

The CDF and D0 detectors have central trackers surrounded by hermetic calorimeters and muon detectors and are designed to study the products of $1.96 \mathrm{TeV}$ protonantiproton collisions [40,41]. Most searches combined here use the complete Tevatron data sample, which corresponds to up to $10 \mathrm{fb}^{-1}$ depending on the experiment and the search channel, after data-quality requirements. The online event selections (triggers) rely on fast reconstruction of combinations of high- $p_{T}$ lepton candidates, jets, and missing transverse energy $\left(\mathscr{E}_{T}\right)$, defined below. To maximize sensitivity, all events satisfying any trigger requirement from the complete suite of triggers used for data taking are considered whenever possible. For instance, while most of the $H \rightarrow W^{+} W^{-}$candidate events are selected by single-lepton and dilepton triggers, a gain in efficiency of up to $20 \%$, depending on the channel, is achieved by including events that pass lepton + jets and lepton $+\mathbb{E}_{T}$ triggers. 
TABLE I. Luminosities, explored mass ranges, and references for the different processes and final states $\left(\ell=e\right.$ or $\mu$, and $\tau_{\text {had }}$ denotes a hadronic tau-lepton decay) for the CDF analyses. The generic labels " $1 \times$, , " $2 \times$, , " $3 \times$," and " $4 \times$ " refer to separations based on lepton or photon categories. The analyses are grouped in five categories, corresponding to the Higgs boson decay mode to which the analysis is most sensitive: $H \rightarrow b \bar{b}, H \rightarrow W^{+} W^{-}, H \rightarrow \tau^{+} \tau^{-}, H \rightarrow \gamma \gamma$, and $H \rightarrow Z Z$.

\begin{tabular}{|c|c|c|c|c|}
\hline Channel & & $\begin{array}{l}\text { Luminosity } \\
\qquad\left(\mathrm{fb}^{-1}\right)\end{array}$ & $\begin{array}{l}m_{H} \text { range } \\
\left(\mathrm{GeV} / c^{2}\right)\end{array}$ & Reference \\
\hline$W H \rightarrow \ell \nu b \bar{b}$ 2-jet channels $4 \times(5 b$-tag categories $)$ & & 9.45 & $90-150$ & [42] \\
\hline$W H \rightarrow \ell \nu b \bar{b} 3$-jet channels $3 \times(2 b$-tag categories $)$ & & 9.45 & $90-150$ & [42] \\
\hline$Z H \rightarrow \nu \bar{\nu} b \bar{b}(3 b$-tag categories) & & 9.45 & $90-150$ & [43] \\
\hline$Z H \rightarrow \ell^{+} \ell^{-} b \bar{b}$ 2-jet channels $2 \times(4 b$-tag categories $)$ & $H \rightarrow b \bar{b}$ & 9.45 & $90-150$ & [44] \\
\hline $\mathrm{ZH} \rightarrow \ell^{+} \ell^{-} b \bar{b} 3$-jet channels $2 \times(4 b$-tag categories $)$ & & 9.45 & $90-150$ & [44] \\
\hline$W H+Z H \rightarrow j j b \bar{b}$ (2b-tag categories) & & 9.45 & $100-150$ & [45] \\
\hline$t \bar{t} H \rightarrow W^{+} b W^{-} \bar{b} b \bar{b}(4$ jets, 5 jets, $\geq 6$ jets $) \times(5 b$-tag categories $)$ & & 9.45 & $100-150$ & {$[46]$} \\
\hline$H \rightarrow W^{+} W^{-} 2 \times(0$ jets $)+2 \times(1$ jet $)+1 \times(\geq 2$ jets $)+1 \times\left(\right.$ low $\left.-m_{\ell \ell}\right)$ & & 9.7 & $110-200$ & [47] \\
\hline$H \rightarrow W^{+} W^{-}\left(e-\tau_{\text {had }}\right)+\left(\mu-\tau_{\text {had }}\right)$ & & 9.7 & $130-200$ & [47] \\
\hline$W H \rightarrow W W^{+} W^{-}$(same-sign leptons) + (trileptons) & $H \rightarrow W^{+} W^{-}$ & 9.7 & $110-200$ & [47] \\
\hline$W H \rightarrow W W^{+} W^{-}$(trileptons with $1 \tau_{\text {had }}$ ) & & 9.7 & $130-200$ & [47] \\
\hline$Z H \rightarrow Z W^{+} W^{-}$(trileptons with 1 jet, $\geq 2$ jets) & & 9.7 & $110-200$ & [47] \\
\hline$H \rightarrow \tau^{+} \tau^{-}(1$ jet $)+(\geq 2$ jets $)$ & $H \rightarrow \tau^{+} \tau^{-}$ & 6.0 & $100-150$ & [48] \\
\hline$H \rightarrow \gamma \gamma 1 \times(0$ jet $)+1 \times(\geq 1$ jet $)+3 \times($ all jets $)$ & $H \rightarrow \gamma \gamma$ & 10.0 & $100-150$ & [49] \\
\hline$H \rightarrow Z Z$ (four leptons) & $H \rightarrow Z Z$ & 9.7 & $120-200$ & [50] \\
\hline
\end{tabular}

High-quality electron candidates are identified by associating charged-particle tracks with deposits of energy in the electromagnetic calorimeters when both measurements are available. High-quality muon candidates are identified by associating tracks with hits in the muon detectors surrounding the calorimeters in the CDF and D0 detectors. Lepton candidates are categorized based on the quality of the contributing measurements. Tight selection requirements yield samples of leptons with low background rates from hadrons or jets of hadrons misidentified as leptons. Looser requirements are designed to increase the acceptance for lepton candidates with poorly measured or partially missing information, with resulting higher rates for backgrounds. To optimize the sensitivity of the combined results, events that are selected with high-quality leptons are analyzed separately from those with low-quality leptons.

TABLE II. Luminosities, explored mass ranges, and references for the different processes and final states $\left(\ell=e\right.$ or $\mu$, and $\tau_{\text {had }}$ denotes a hadronic tau-lepton decay) for the D0 analyses. The generic labels " $1 \times$, , " $2 \times$, , " $3 \times$," and " $4 \times$ " refer to separations based on lepton, photon, or background characterization categories. The analyses are grouped in four categories, corresponding to the Higgs boson decay mode to which the analysis is most sensitive: $H \rightarrow b \bar{b}, H \rightarrow W^{+} W^{-}, H \rightarrow \tau^{+} \tau^{-}$, and $H \rightarrow \gamma \gamma$.

\begin{tabular}{|c|c|c|c|c|}
\hline Channel & & $\begin{array}{c}\text { Luminosity } \\
\left(\mathrm{fb}^{-1}\right)\end{array}$ & $\begin{array}{l}m_{H} \text { range } \\
\left(\mathrm{GeV} / c^{2}\right)\end{array}$ & Reference \\
\hline$W H \rightarrow \ell \nu b \bar{b} 2$-jet channels $2 \times(4 b$-tag categories $)$ & \multirow{4}{*}{$H \rightarrow b \bar{b}$} & 9.7 & $90-150$ & {$[51,52]$} \\
\hline$W H \rightarrow \ell \nu b \bar{b} 3$-jet channels $2 \times(4 b$-tag categories $)$ & & 9.7 & $90-150$ & {$[51,52]$} \\
\hline$Z H \rightarrow \nu \bar{\nu} b \bar{b}(2 b-$ tag categories $)$ & & 9.5 & $100-150$ & [53] \\
\hline$Z H \rightarrow \ell^{+} \ell^{-} b \bar{b} 2 \times(2 b-\mathrm{tag}) \times(4$ lepton categories $)$ & & 9.7 & $90-150$ & {$[54,55]$} \\
\hline$H \rightarrow W^{+} W^{-} \rightarrow \ell^{ \pm} \nu \ell^{\mp} \nu 2 \times(0$ jets, 1 jet, $\geq 2$ jets $)$ & \multirow{6}{*}{$H \rightarrow W^{+} W^{-}$} & 9.7 & $115-200$ & {$[56]$} \\
\hline$H+X \rightarrow W^{+} W^{-} \rightarrow \mu^{\mp} \nu \tau_{\mathrm{had}}^{ \pm} \nu(3 \tau$ categories $)$ & & 7.3 & $115-200$ & [57] \\
\hline$H \rightarrow W^{+} W^{-} \rightarrow \ell \bar{\nu} j j 2 \times(2 b$-tag categories $) \times(2$ jets, 3 jets $)$ & & 9.7 & $100-200$ & [52] \\
\hline$V H \rightarrow e^{ \pm} \mu^{ \pm}+X$ & & 9.7 & $100-200$ & [58] \\
\hline$V H \rightarrow \ell \ell \ell+X(\mu \mu e, 3 \times e \mu \mu)$ & & 9.7 & $100-200$ & [58] \\
\hline$V H \rightarrow \ell \bar{\nu} j j j j 2 \times(\geq 4$ jets $)$ & & 9.7 & $100-200$ & {$[52]$} \\
\hline$V H \rightarrow \tau_{\text {had }} \tau_{\text {had }} \mu+X(3 \tau$ categories $)$ & \multirow{2}{*}{$H \rightarrow \tau^{+} \tau^{-}$} & 8.6 & $100-150$ & {$[58]$} \\
\hline$H+X \rightarrow \ell^{ \pm} \tau_{\text {had }}^{\mp} j j 2 \times(3 \tau$ categories $)$ & & 9.7 & $105-150$ & [59] \\
\hline$H \rightarrow \gamma \gamma$ (4 categories) & $H \rightarrow \gamma \gamma$ & 9.6 & $100-150$ & {$[60]$} \\
\hline
\end{tabular}


Jets are clustered from energy deposits in the electromagnetic and hadronic calorimeters and, in some analyses, combine information from charged particle tracks to improve purity or energy resolution. The transverse energy vector $\vec{E}_{T}$ of a calorimeter energy deposit is $E \sin \theta \hat{n}$, where $E$ is the measured energy, $\theta$ is the angle with respect to the proton beam axis of a line drawn from the collision point to the energy deposit, and $\hat{n}$ is a unit vector in the plane perpendicular to the beam pointing along that line. The missing transverse energy $\mathscr{E}_{T}$ is the magnitude of the vector opposite to the sum of the $\vec{E}_{T}$ vectors measured in the calorimeter, after propagation of all corrections to the calorimetric objects and for identified muons (which deposit only small amounts of energy in the calorimeters) contributing to the signal topology. Further details of the object reconstruction algorithms used in the Higgs boson searches can be found in the references for the individual analyses (see Tables I and II).

\section{EVENT SELECTION}

Event selections are similar in the CDF and D0 analyses, typically consisting of a preselection based on event topology and kinematics. Multivariate analysis (MVA) techniques [61] are used to combine several discriminating variables into a single final discriminant that is used in the statistical interpretation to compute upper limits, $p$-values, and fitted cross sections. Each channel is divided into exclusive subchannels according to various lepton, jet multiplicity, and $b$-tagging characterization criteria. This procedure groups events with similar signal-to-background ratios to optimize the overall sensitivity. Such subdivision allows, for example, the efficient use of poorly reconstructed leptons or those in the forward region, the exploitation of the different dominant signal and backgrounds when training the MVAs separately in each subchannel, or reduction of the impact of systematic uncertainties. The MVAs are trained separately at each value of $m_{H}$ in their respective mass ranges, in $5 \mathrm{GeV} / c^{2}$ steps.

For the analyses exploiting the $H \rightarrow b \bar{b}$ decay, $b$-tagging and dijet mass resolution are of great importance. Both collaborations have developed multivariate approaches to maximize the performance of the $b$-tagging algorithms. The CDF $b$-tagging algorithm is based on an MVA [62] and, depending on the chosen operating point, provides $b$-tagging efficiencies of $50 \%-70 \%$ with misidentification rates for light ( $u, d, s$, and gluon) jets of $0.5 \%-6 \%$. In the D0 analyses, the MVA builds and improves upon the previous neural network $b$-tagger $[53,63]$ and achieves identification efficiencies of about $80 \%(50 \%)$ for $b$ jets for a light jet misidentification rate of about $10 \%(0.5 \%)$.

The decay width of the SM Higgs boson is predicted to be much smaller than the experimental dijet mass resolution, which is typically $15 \%$ of the mean reconstructed mass. A SM Higgs boson signal would appear as a broad enhancement in the reconstructed di- $b$-jet mass distribution. The CDF and D0 Collaborations search for $H \rightarrow b \bar{b}$ produced in association with a leptonically decaying $W$ boson, or a leptonically or invisibly decaying $Z$ boson. $\mathrm{CDF}$ also contributes searches for $W H+Z H \rightarrow j j b \bar{b}$ and $t \bar{t} H \rightarrow t \bar{t} b \bar{b}$, where in the latter case one of the top quarks decays to a leptonically decaying $W$ boson.

Both collaborations search for the $H \rightarrow W^{+} W^{-}$signal in which both $W$ bosons decay leptonically by selecting events with large missing transverse energy and two oppositely charged, isolated leptons. The presence of neutrinos in the final state prevents reconstruction of the Higgs boson mass. Other observables are used for separating the signal from background. For example, the azimuthal angle between the leptons in signal events is smaller on average than that in background events due to the scalar nature of the Higgs boson and parity violation in $W^{ \pm}$decays. Furthermore, the missing transverse momentum is larger and the total transverse energy of the jets is lower than they are typically in background events. The D0 Collaboration also includes channels in which one of the $W$ bosons in the $H \rightarrow W^{+} W^{-}$process decays leptonically and the other hadronically.

Although the primary sensitivity at low mass $\left(m_{H} \leq\right.$ $\left.130 \mathrm{GeV} / c^{2}\right)$ is provided by the $H \rightarrow b \bar{b}$ analyses and at high mass $\left(m_{H}>130 \mathrm{GeV} / c^{2}\right)$ by the $H \rightarrow W^{+} W^{-}$ analyses, significant additional sensitivity is achieved by the inclusion of other channels. Both collaborations contribute analyses searching for Higgs bosons decaying into trilepton final states, tau-lepton pairs, and diphoton pairs. The full list of channels included is shown in Tables I and II which summarize, for the CDF and D0 analyses respectively, the integrated luminosities, the Higgs boson mass ranges over which the searches are performed, and references to further details for each analysis.

\section{CANDIDATE DISTRIBUTION}

The number of contributing channels is large, and several different kinds of discriminating variables are used. Visual comparison of the observed data with the predictions is challenging in some of the subchannels due to low data counts. For a more robust comparison, we display the data from all the subchannels together, aggregating bins with similar signal-to-background ratios $(s / b)$ from all contributing subchannels. We collect the signal predictions, the background predictions, and the data in narrow bins of $s / b$, summing the contributions from bins in the final discriminant histograms in the subchannels. A fit of the background model (see Sec. VI) to the data is performed before this aggregation procedure, in order to provide the best prediction for the background model in bins with the highest sensitivity. The classification of analysis events according to their $s / b$ preserves the importance of each of the events in the histogram, to the extent that they are not added to other events that are selected with different $s / b$. This representation of the data is not used to compute the final results, since the distribution indiscriminately sums unrelated backgrounds which are fit separately. It does, 


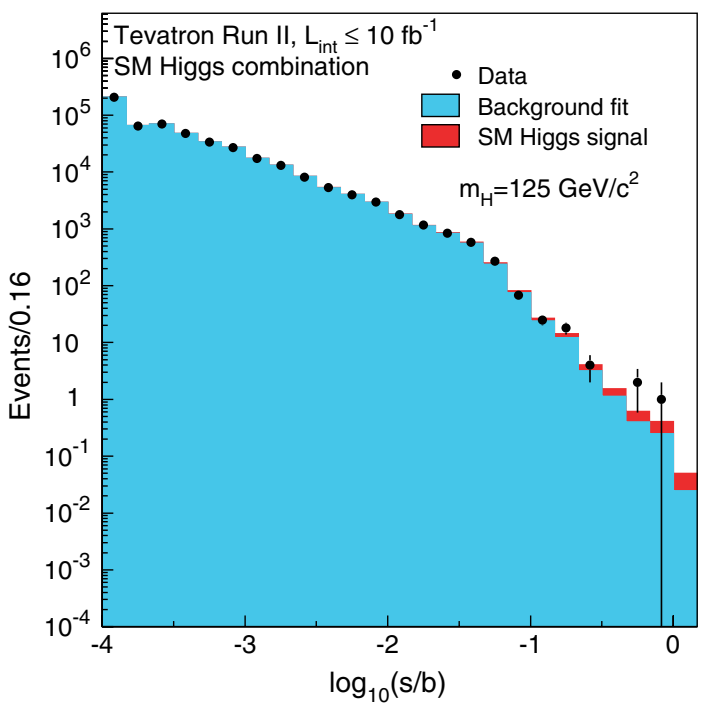

FIG. 1 (color online). Distribution of $\log _{10}(s / b)$, for the data from all contributing Higgs boson search channels from CDF and D0, for $m_{H}=125 \mathrm{GeV} / c^{2}$. The data are shown with points, and the expected signal is shown stacked on top of the backgrounds, which are fit to the data within their systematic uncertainties. The error bars shown on the data correspond in each bin to the square root of the observed data count. Underflows and overflows are collected into the leftmost and rightmost bins, respectively.

however, provide a guide to how much individual events contribute to the results and how well the signal is separated from backgrounds in the combined search. The resulting distribution of $\log _{10}(s / b)$ is shown for $m_{H}=125 \mathrm{GeV} / c^{2}$ in Fig. 1, demonstrating agreement with background over 5 orders of magnitude.

\section{STATISTICAL TECHNIQUES}

The results are interpreted using both Bayesian and modified frequentist techniques, separately at each value of $m_{H}$, as was done previously $[12,13,64]$. The two methods yield results that are numerically consistent; limits on the Higgs boson production rate typically agree within $5 \%$ at each value of $m_{H}$, and with a $1 \%$ deviation when averaged over all positive and negative departures. For simplicity, when summarizing the results, we quote one set of values as the default, and the a priori decision made for the earlier Tevatron combinations to use the Bayesian method is retained here. Both methods use the distributions of the final discriminants and not only the total event counts passing selection requirements.

Each of the techniques is built on a combined likelihood [including prior probability densities on systematic uncertainties, $\pi(\vec{\theta})$ ] based on the product of likelihoods for the individual channels, each of which is a product over histogram bins,

$$
\mathcal{L}(R, \vec{s}, \vec{b} \mid \vec{n}, \vec{\theta}) \times \pi(\vec{\theta})=\prod_{i=1}^{N_{\mathrm{C}} \prod_{j=1}^{N_{\mathrm{bins}}}} \mu_{i j}^{n_{i j}} \frac{e^{-\mu_{i j}}}{n_{i j} !} \times \prod_{k=1}^{n_{\mathrm{sys}}} e^{-\theta_{k}^{2} / 2},
$$

where the first product is over the number of channels $\left(N_{\mathrm{C}}\right)$ and the second product is over histogram bins containing $n_{i j}$ events, binned in ranges of the final discriminants used for the individual analyses. The predictions for the bin contents are $\mu_{i j}=R \times s_{i j}(\vec{\theta})+b_{i j}(\vec{\theta})$ for channel $i$ and histogram bin $j$, where $s_{i j}$ and $b_{i j}$ represent the expected SM signal and background in the bin, and $R$ is a scaling factor applied to the signal. By scaling all signal contributions by the same factor we assume that the relative contributions of the different processes at each $m_{H}$ are as predicted by the SM. Systematic uncertainties are parametrized by the dependence of $s_{i j}$ and $b_{i j}$ on $\vec{\theta}$. Each of the $n_{\text {sys }}$ components of $\vec{\theta}, \theta_{k}$ corresponds to a single independent source of systematic uncertainty scaled by its standard deviation, and each parameter may affect the predictions of several sources of signal and background in different channels, thus accounting for correlations. Gaussian prior densities are assumed for the nuisance parameters, truncated to ensure that no prediction is negative.

In the Bayesian calculation, we assume a uniform prior probability density for non-negative values of $R$ and integrate the likelihood function multiplied by prior densities for the nuisance parameters to obtain the posterior density for $R$. The observed 95\% credibility level upper limit on $R$, $R_{95}^{\mathrm{obs}}$, is the value of $R$ such that the integral of the posterior density of $R$ from zero to $R_{95}^{\mathrm{obs}}$ corresponds to $95 \%$ of the integral of $R$ from zero to infinity. The expected distribution of $R_{95}$ is computed in an ensemble of simulated experimental outcomes assuming no signal is present. In each simulated outcome, random values of the nuisance parameters are drawn from their prior densities. A combined measurement of the cross section for Higgs boson production times the branching fraction $\mathcal{B}(H \rightarrow X X)$, in units of the SM production rate, is given by $R^{\text {fit }}$, which is the value of $R$ that maximizes the posterior density. The $68 \%$ credibility interval, which corresponds to 1 standard deviation (s.d.), is quoted as the smallest interval containing $68 \%$ of the integral of the posterior.

We also perform calculations with the modified frequentist technique $\mathrm{CL}_{\mathrm{S}}$ [64], using a log-likelihood ratio $(\mathrm{LLR})$ as the test statistic: $\mathrm{LLR}=-2 \ln \frac{p(\text { data } \mid s+b)}{p(\text { data } \mid b)}$, where $p($ data $\mid s+b)$ and $p($ data $\mid b)$ are the probabilities that the data (either simulated or experimental data) are drawn from distributions predicted under the signal-plusbackground and background-only hypotheses, respectively. The probabilities $p$ are computed using the best-fit values of the parameters $\theta_{k}$, separately for each of the two hypotheses [65]. The use of these fits extends the procedure used at LEP [66], improving the sensitivity when the expected signals are small and the uncertainties on the backgrounds are large. The $\mathrm{CL}_{\mathrm{s}}$ technique involves computing two $p$-values, $\mathrm{CL}_{\mathrm{b}}=p\left(\mathrm{LLR} \geq \mathrm{LLR}_{\mathrm{obs}} \mid b\right)$, where $L_{L R}$ obs is the value of the test statistic computed for the data, and $\mathrm{CL}_{\mathrm{s}+\mathrm{b}}=p\left(\operatorname{LLR} \geq \operatorname{LLR}_{\mathrm{obs}} \mid s+b\right)$. To 
compute limits, we use the ratio of $p$-values, $\mathrm{CL}_{\mathrm{s}}=$ $\mathrm{CL}_{\mathrm{s}+\mathrm{b}} / \mathrm{CL}_{\mathrm{b}}$. If $\mathrm{CL}_{\mathrm{s}}<0.05$ for a particular choice of the signal-plus-background hypothesis, parametrized by the signal scale factor $R$, that hypothesis is excluded at least at the $95 \%$ C.L. The value of $R_{95}^{\text {obs }}$ in the $\mathrm{CL}_{\mathrm{s}}$ method is the smallest value of $R$ excluded at the 95\% C.L. The expected limit is computed using the median LLR value expected in the background-only hypothesis. Systematic uncertainties are included by fluctuating the nuisance parameters $\vec{\theta}$ within their prior distributions when generating the pseudoexperiments used to compute $\mathrm{CL}_{\mathrm{s}+\mathrm{b}}$ and $\mathrm{CL}_{\mathrm{b}}$.

In this framework, a second estimate of the signal rate, $R_{\text {profile }}^{\mathrm{fit}}$, is computed, maximizing the likelihood as a function of the unconstrained signal rate $R$ and the nuisance parameters $\theta_{k}$. This estimate of the combined signal rate may differ from the Bayesian calculation of $R^{\mathrm{fit}}$ when the likelihood function deviates from a Gaussian form, since the best fit depends on the likelihood near the maximum and the Bayesian calculation integrates over all values of the nuisance parameters which result in positive signal and background rates in all histogram bins.

\section{SYSTEMATIC UNCERTAINTIES}

Systematic uncertainties are evaluated for each final state, background, and signal process. Uncertainties that modify only the normalization and uncertainties that change the shape of the final discriminant distribution are included. To study the shape uncertainties on the distributions of the final discriminants, the relevant parameter is varied within 1 standard deviation of its uncertainty and the full analysis repeated using the modified distribution. For example, for the jet energy scale and resolution, the parameters of the energy scale and resolution are varied within 1 s.d. of their uncertainties and the analysis carried out using the kinematic distributions of the modified jets, also including the changes in sample composition resulting from the change in the jet energy parameters. No retraining of the MVAs is performed during the propagation of systematic uncertainties to the distributions of the discriminants. Correlations between signal and background, across different channels within an experiment and across the two experiments, are taken into account. Full details on the treatment of the systematic uncertainties in the individual channels can be found in the relevant references.

The uncertainties on the inclusive signal production cross sections are estimated from the variations in the factorization and renormalization scale, which include the impact of uncalculated higher-order corrections, uncertainties due to PDFs, and the dependence on the strong coupling constant, $\alpha_{s}$, as recommended by the PDF4LHC working group $[67,68]$. The resulting uncertainties on the inclusive $\mathrm{VH}$ and VBF production rates are taken to be $7 \%$ and $5 \%$, respectively [20]. Uncertainties on the branching fractions are taken from Ref. [69].
For analyses focusing on $g g \rightarrow H$ production that divide events into categories based on the number of reconstructed jets, the uncertainties associated with the renormalization and factorization scale are estimated following Ref. [70]. By propagating the uncorrelated uncertainties of the NNLL inclusive [18,19], NLO $\geq 1$ jet [68], and NLO $\geq 2$ jets [71] cross sections to the exclusive $g g \rightarrow H+0$ jet, $\geq 1$ jet, and $\geq 2$ jets rates, an uncertainty matrix containing correlated and uncorrelated uncertainty contributions between exclusive jet categories is obtained. The total uncertainty on $g g \rightarrow H$ production originating from these contributions varies from $10 \%$ to $35 \%$ in individual channels depending on the number of jets in the final state. The PDF uncertainties are evaluated following Refs. $[18,68]$.

Significant sources of uncertainty for all analyses are the integrated luminosities used to normalize the expected signal yield and MC-based backgrounds, and the cross sections for the simulated backgrounds. For the former, uncertainties of $6 \%$ (CDF) and 6.1\% (D0) are used, with $4 \%$ arising from the inelastic $p \bar{p}$ cross section which is taken to be $100 \%$ correlated between CDF and D0. Crosssection uncertainties of $6 \%$ and $7 \%$ are used for diboson and $t \bar{t}$ production, respectively. The uncertainty on the expected multijet background in each channel is dominated by the statistics of the data sample from which it is estimated and varies from $10 \%$ to $30 \%$.

Sources of systematic uncertainty that affect both the normalization and the shape of the final discriminant distribution include jet energy scale (1-4)\%, jet energy resolution (1-3)\%, lepton identification, trigger efficiencies, and $b$ tagging. Uncertainties on lepton identification and trigger efficiencies range from $2 \%$ to $6 \%$ and are applied to both the signal and MC-based background predictions. These uncertainties are estimated from data-based methods separately by CDF and D0, and differ based on lepton flavor and identification category. The $b$-tag efficiencies and mistag rates are similarly constrained by auxiliary data samples, such as inclusive jet data or $t \bar{t}$ events. The uncertainty on the per-jet $b$-tag efficiency is approximately $4 \%$, and the mistag uncertainties vary between $7 \%$ and $15 \%$.

For the analyses targeting the $H \rightarrow b \bar{b}$ decay, the largest sources of uncertainty on the dominant backgrounds are the rates of $V+$ heavy flavor jets, which are typically (20-30)\% of the predicted values. Using constraints from the data, the uncertainties on these rates are typically $8 \%$ or less. The data samples in the $V+$ jets selections prior to $b$ tagging are used as control samples to constrain systematic uncertainties in the MC modeling of the energies and angles of jets. Any residual discrepancy coming from the difference between light- and heavy-flavor components is shown to be smaller than the systematic uncertainties associated with the generator or the correction procedures themselves.

A total of 326 independent sources of systematic uncertainty are included in the combination of the Higgs boson search results at $m_{H}=125 \mathrm{GeV} / c^{2}$, not including the 
independent uncertainties in each bin of each template from limited Monte Carlo (or data) statistics. The uncertainties that are considered correlated between CDF and D0 are those on the differential and inclusive theoretical production cross-section predictions for the Higgs boson signals (itemized by $\mathrm{PDF}+\alpha_{s}$ and scales), the Higgs boson decay branching fractions, the $t \bar{t}$, single top, and diboson background processes, and the correlated part of the luminosity estimate. All other uncertainties are associated with parameters whose central values are estimated using techniques specific to the experiments and the analysis channels. We consider these uncorrelated so as not to extrapolate fit information improperly from one channel or experiment to another where the central value or the uncertainty scale may be different.

\section{RESULTS-STANDARD MODEL INTERPRETATION}

\section{A. Diboson production}

To validate our background modeling and methodology, independent measurements of SM diboson production in the same final states used for the SM Higgs searches are carried out. The high-mass analyses measure $p \bar{p} \rightarrow V V^{\prime}$ cross sections, while the low-mass analyses target $V Z(\rightarrow b \bar{b})$ production. The data sample, reconstruction, process modeling, uncertainties, and subchannel divisions are identical to those of the SM Higgs boson searches. However, discriminant functions are trained to distinguish the contributions of SM diboson production from those of other backgrounds, and potential contributions from Higgs boson production are not considered. By way of illustration, below, we focus on $V Z$ production.

The NLO SM cross section for $V Z$ production times the branching fraction of $Z \rightarrow b \bar{b}$ is $0.68 \pm 0.05 \mathrm{pb}[33,72]$. This is about 6 times larger than the $0.12 \pm 0.01 \mathrm{pb}[20,25]$ cross section times branching fraction of $H(\rightarrow b \bar{b}) V$ for a $125 \mathrm{GeV} / c^{2} \mathrm{SM}$ Higgs boson, but the associated background is larger, due to the distribution of the dijet invariant mass in the $V+$ jets events. $W W$ production is considered as background. The measured cross section, using the MVA discriminants, for $V Z$ is $3.0 \pm 0.6$ (stat) \pm 0.7 (syst) $\mathrm{pb}$ whereas the $\mathrm{SM}$ prediction is $4.4 \pm 0.3 \mathrm{pb}$ [33]. The combined background-subtracted dijet-mass distribution for the $V Z$ analysis is shown in Fig. 2 for illustration. The $V Z$ signal and the background contributions are fit to the data, and the fitted background is then subtracted. Also shown is the contribution expected from a SM Higgs boson with $m_{H}=125 \mathrm{GeV} / c^{2}$. The $V V^{\prime}$ boson cross sections measured by the high-mass analyses are likewise in good agreement with SM predictions [15,73].

\section{B. Higgs boson combination using all decay modes}

For the search for the Higgs boson, the results produced by the multivariate analyses can be visualized by combining the histograms of the final discriminants, adding the

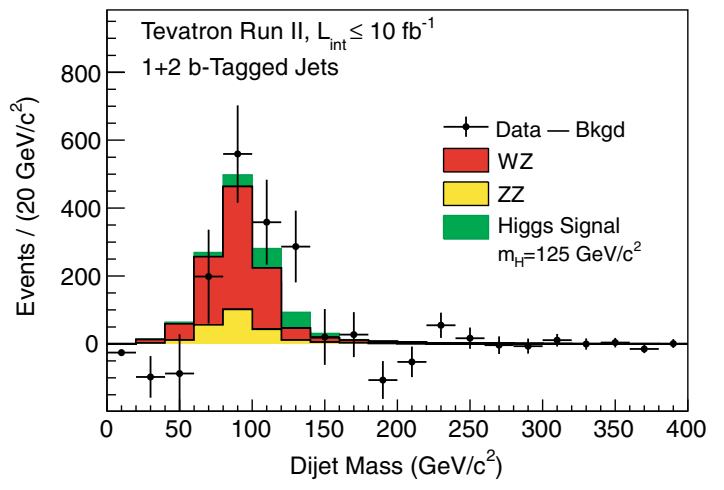

FIG. 2 (color online). Background-subtracted distribution of the reconstructed dijet mass, summed over CDF's and D0's channels contributing to the $V Z$ analysis. The $V Z$ signal and the background contributions are fit to the data, and the fitted background is subtracted. The fitted $V Z$ and expected SM Higgs $\left(m_{H}=125 \mathrm{GeV} / c^{2}\right)$ contributions are shown with filled histograms. The error bars shown on the data points correspond in each bin to the square root of the sum of the expected signal and background yields.

contents of bins with similar signal-to-background ratio $(s / b)$ as shown in Fig. 1. Figure 3 shows the signal expectation and the data with the background subtracted, as a function of the $s / b$ of the collected bins, for the combined search for a Higgs boson with mass $m_{H}=125 \mathrm{GeV} / c^{2}$. The background model is fit to the data, allowing the nuisance parameters to vary within their constraints. The uncertainties on the background predictions in each bin are

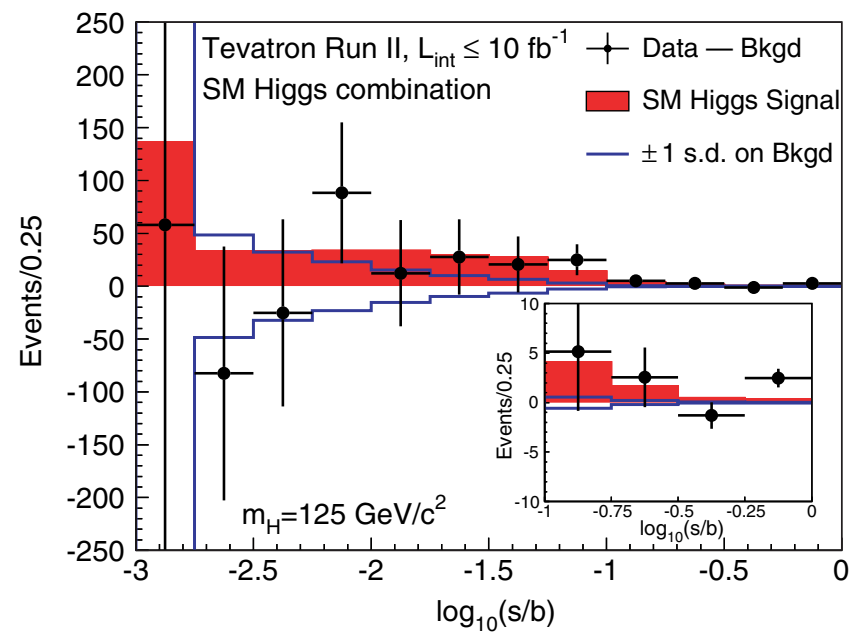

FIG. 3 (color online). Background-subtracted distribution of the discriminant histograms, summed for bins with similar signal-to-background ratio $(s / b)$ over all contributing Higgs boson search channels from $\mathrm{CDF}$ and $\mathrm{D} 0$, for $m_{H}=$ $125 \mathrm{GeV} / c^{2}$. The background is fit to the data, and the uncertainty on the background, shown with the unfilled histogram, is after the fit. The signal model, scaled to the SM expectation, is shown with a filled histogram. The error bars shown on the data points correspond in each bin to the square root of the sum of the expected signal and background yields. 


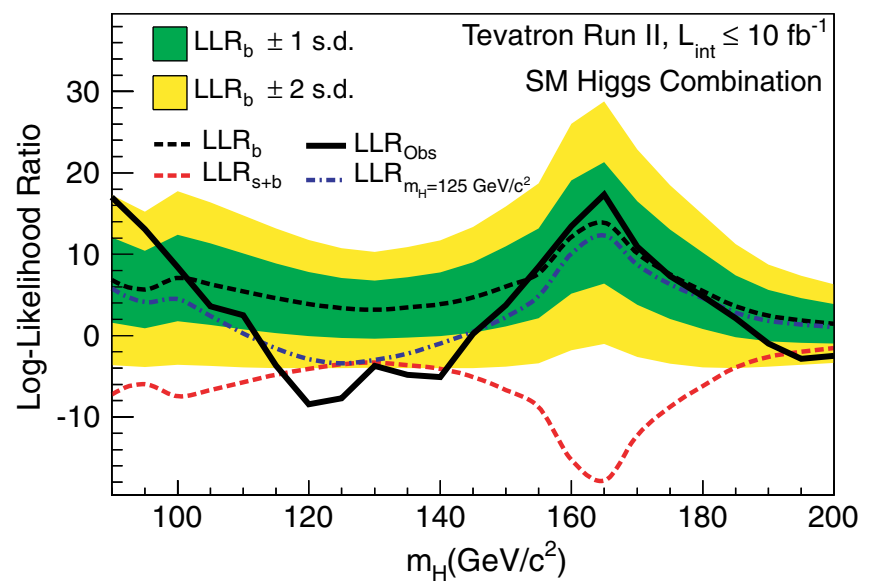

FIG. 4 (color online). LLR as a function of Higgs boson mass for all of CDF's and D0's SM Higgs boson searches in all decay modes combined. The solid line shows the observed LLR values, the dark long-dashed line shows the median expectation assuming no Higgs boson signal is present, and the dark- and light-shaded bands correspond, respectively, to the regions encompassing 1 and 2 s.d. fluctuations around the background-only expectation. The red long-dashed line shows the median expectation assuming a SM Higgs boson signal is present at each value of $m_{H}$ in turn. The blue short-dashed line shows the median expected LLR assuming the SM Higgs boson is present at $m_{H}=125 \mathrm{GeV} / c^{2}$. those after the fit. An excess of events in the highest $s / b$ bins relative to the background-only expectation is observed.

Figure 4 displays the LLR distributions for the combined analyses as functions of $m_{H}$. Included are the median of the LLR distributions for the background-only hypothesis $\left(\operatorname{LLR}_{b}\right)$, the signal-plus-background hypothesis $\left(\mathrm{LLR}_{s+b}\right)$, and the observed value for the data $\left(\mathrm{LLR}_{\mathrm{obs}}\right)$. For mass hypotheses of $95 \mathrm{GeV} / c^{2}$ and less, fewer channels are available for combination, giving rise to the behavior of the limits shown. The shaded bands represent the 1 and 2 s.d. departures for $\mathrm{LLR}_{b}$ centered on the median. These results are listed in Table III. The separation between the medians of the $\mathrm{LLR}_{b}$ and $\mathrm{LLR}_{s+b}$ distributions provides a measure of the discriminating power of the search. The widths of the 1- and 2-s.d. $\mathrm{LLR}_{b}$ bands indicate the width of the $\mathrm{LLR}_{b}$ distribution, assuming no signal and that fluctuations originate from statistical fluctuations and systematic effects only. The value of $L_{L R}$ obs relative to $L_{L R}+b$ and $\mathrm{LLR}_{b}$ indicates whether the data distribution more closely resembles the distributions expected if a signal is present (i.e., the $\mathrm{LLR}_{s+b}$ distribution, which is negative by construction) or only background is present. The significance of departures of $\mathrm{LLR}_{\mathrm{obs}}$ from $\mathrm{LLR}_{b}$ can be evaluated by the width of the $\operatorname{LLR}_{b}$ bands. The separation of the median signal-plus-background and background-only hypotheses is about 2 s.d., or greater, for Higgs boson

TABLE III. LLR values obtained from the combination of all of CDF's and D0's Higgs boson search channels using the $\mathrm{CL}_{\mathrm{s}}$ method.

\begin{tabular}{lrrrrrrr}
\hline \hline$m_{H}\left(\mathrm{GeV} / c^{2}\right)$ & $\mathrm{LLR}_{\mathrm{obs}}$ & $\mathrm{LLR}_{s+b}$ & $\mathrm{LLR}_{b}^{-2 \sigma}$ & $\mathrm{LLR}_{b}^{-1 \sigma}$ & $\mathrm{LLR}_{b}$ & $\mathrm{LLR}_{b}^{+1 \sigma}$ & $\mathrm{LLR}_{b}^{+2 \sigma}$ \\
\hline 90 & 17.02 & -7.24 & 17.31 & 12.08 & 6.84 & 1.61 & -3.62 \\
95 & 13.07 & -5.96 & 15.21 & 10.44 & 5.68 & 0.91 & -3.85 \\
100 & 8.39 & -7.44 & 17.73 & 12.40 & 7.08 & 1.76 & -3.56 \\
105 & 3.62 & -6.69 & 16.38 & 11.35 & 6.32 & 1.29 & -3.74 \\
110 & 2.53 & -5.73 & 14.79 & 10.12 & 5.45 & 0.78 & -3.89 \\
115 & -3.67 & -4.81 & 13.17 & 8.88 & 4.59 & 0.31 & -3.98 \\
120 & -8.44 & -4.09 & 11.76 & 7.82 & 3.88 & -0.06 & -4.00 \\
125 & -7.72 & -3.52 & 10.76 & 7.07 & 3.39 & -0.29 & -3.97 \\
130 & -3.74 & -3.30 & 10.31 & 6.74 & 3.18 & -0.39 & -3.95 \\
135 & -4.81 & -3.64 & 10.89 & 7.17 & 3.45 & -0.26 & -3.98 \\
140 & -5.08 & -4.09 & 11.72 & 7.79 & 3.86 & -0.07 & -4.00 \\
145 & 0.20 & -5.07 & 13.35 & 9.02 & 4.69 & 0.36 & -3.97 \\
150 & 3.72 & -6.68 & 15.87 & 10.95 & 6.04 & 1.12 & -3.79 \\
155 & 8.44 & -8.80 & 18.72 & 13.18 & 7.65 & 2.12 & -3.41 \\
160 & 13.45 & -15.25 & 26.04 & 19.08 & 12.12 & 5.15 & -1.81 \\
165 & 17.33 & -17.81 & 28.76 & 21.31 & 13.87 & 6.42 & -1.03 \\
170 & 10.93 & -12.26 & 22.87 & 16.50 & 10.13 & 3.77 & -2.60 \\
175 & 7.33 & -8.77 & 18.50 & 13.02 & 7.53 & 2.04 & -3.45 \\
180 & 4.86 & -6.17 & 14.87 & 10.18 & 5.50 & 0.81 & -3.88 \\
185 & 2.14 & -3.92 & 11.23 & 7.42 & 3.62 & -0.19 & -3.99 \\
190 & -0.99 & -2.61 & 8.73 & 5.60 & 2.46 & -0.68 & -3.81 \\
195 & -2.83 & -1.98 & 7.34 & 4.60 & 1.87 & -0.87 & -3.60 \\
200 & -2.50 & -1.53 & 6.29 & 3.88 & 1.46 & -0.96 & -3.37 \\
\hline \hline & & & & & & &
\end{tabular}


masses up to $\approx 185 \mathrm{GeV} / c^{2}$. The data are consistent with the background-only hypothesis (the black dashed line) at masses smaller than $\approx 110 \mathrm{GeV} / c^{2}$ and above approximately $145 \mathrm{GeV} / c^{2}$. A slight excess is seen above approximately $195 \mathrm{GeV} / c^{2}$, where our ability to separate the two hypotheses is limited. For $m_{H}$ from 115 to $140 \mathrm{GeV} / c^{2}$, an excess above 2 s.d. in the data with respect to the SM background expectation has an amplitude consistent with the expectation for a standard model Higgs boson (dashed red line). Additionally, the LLR curve under the hypothesis that a SM Higgs boson is present with $m_{H}=125 \mathrm{GeV} / c^{2}$ is shown. This signal-injected-LLR curve has a similar shape to the observed one. While the search for a $125 \mathrm{GeV} / c^{2}$ Higgs boson is optimized to find a Higgs boson of that mass, the excess of events over the SM background estimates also affects the results of Higgs boson searches at other masses. Nearby masses are the most affected, but the expected presence of $H \rightarrow W^{+} W^{-}$decays for a $125 \mathrm{GeV} / c^{2}$ Higgs boson implies a small expected excess in the $H \rightarrow W^{+} W^{-}$searches at all masses due to the poor reconstructed mass resolution in this final state.

The upper limit on SM Higgs boson production as a function of $m_{H}$ is extracted in the range $90-200 \mathrm{GeV} / c^{2}$ in terms of $R_{95}^{\text {obs }}$, the ratio of the observed limit to the predicted SM rate. The ratios of the 95\% C.L. expected and observed limits to the SM cross section using the Bayesian method are shown in Fig. 5 for the combined CDF and D0 analyses. The observed and median-expected ratios are listed for the tested Higgs boson masses in Table IV, as obtained by the Bayesian and the $\mathrm{CL}_{\mathrm{s}}$ methods.

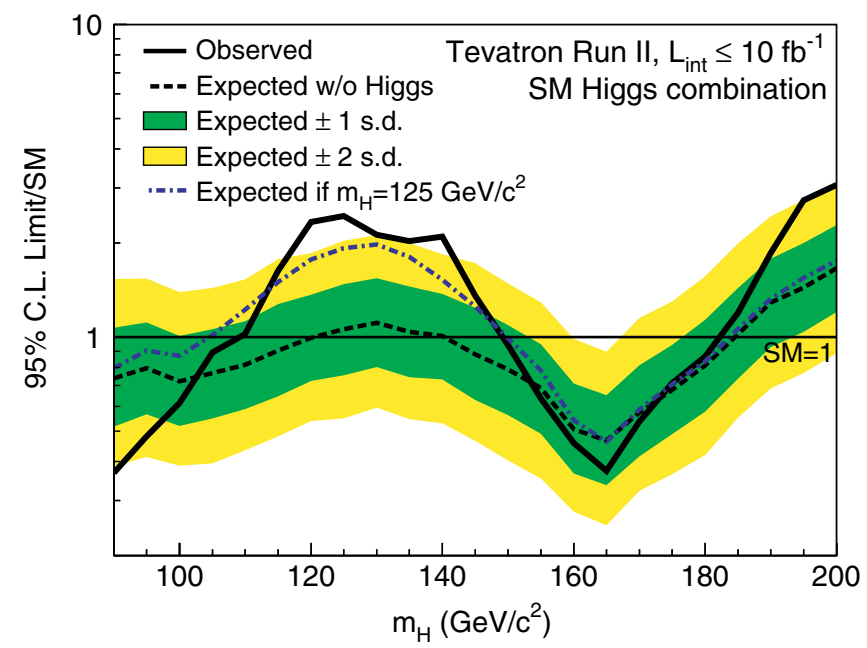

FIG. 5 (color online). Observed and median expected (for the background-only hypothesis) $95 \%$ C.L. Bayesian upper production limits expressed as multiples of the SM cross section as a function of Higgs boson mass for the combined CDF and D0 searches in all decay modes. The dark- and light-shaded bands indicate, respectively, the 1 and 2 s.d probability regions in which the limits are expected to fluctuate in the absence of signal. The blue short-dashed line shows median expected limits assuming the SM Higgs boson is present at $m_{H}=125 \mathrm{GeV} / c^{2}$.
TABLE IV. Ratios of observed and median expected (for the background-only hypothesis) $95 \%$ C.L. upper production limits to the SM cross section as a function of the Higgs boson mass for the combined CDF and D0 searches in all decay modes, obtained using the Bayesian and $\mathrm{CL}_{\mathrm{s}}$ methods.

\begin{tabular}{|c|c|c|c|c|}
\hline \multirow[b]{2}{*}{$m_{H}\left(\mathrm{GeV} / c^{2}\right)$} & \multicolumn{2}{|c|}{ Bayesian } & \multicolumn{2}{|c|}{$\mathrm{CL}_{\mathrm{s}}$} \\
\hline & $R_{95}^{\mathrm{obs}}$ & $R_{95}^{\exp }$ & $R_{95}^{\mathrm{obs}}$ & $R_{95}^{\exp }$ \\
\hline 90 & 0.37 & 0.74 & 0.39 & 0.74 \\
\hline 95 & 0.48 & 0.80 & 0.49 & 0.81 \\
\hline 100 & 0.62 & 0.72 & 0.62 & 0.73 \\
\hline 105 & 0.89 & 0.77 & 0.93 & 0.77 \\
\hline 110 & 1.02 & 0.82 & 1.03 & 0.83 \\
\hline 115 & 1.63 & 0.90 & 1.67 & 0.91 \\
\hline 120 & 2.33 & 1.00 & 2.40 & 0.99 \\
\hline 125 & 2.44 & 1.06 & 2.62 & 1.07 \\
\hline 130 & 2.13 & 1.11 & 2.10 & 1.10 \\
\hline 135 & 2.03 & 1.04 & 2.12 & 1.06 \\
\hline 140 & 2.10 & 1.01 & 2.08 & 1.00 \\
\hline 145 & 1.35 & 0.88 & 1.29 & 0.90 \\
\hline 150 & 0.94 & 0.79 & 0.91 & 0.78 \\
\hline 155 & 0.64 & 0.69 & 0.62 & 0.68 \\
\hline 160 & 0.46 & 0.51 & 0.45 & 0.51 \\
\hline 165 & 0.37 & 0.47 & 0.36 & 0.47 \\
\hline 170 & 0.54 & 0.57 & 0.53 & 0.57 \\
\hline 175 & 0.71 & 0.68 & 0.68 & 0.68 \\
\hline 180 & 0.87 & 0.81 & 0.86 & 0.82 \\
\hline 185 & 1.20 & 1.02 & 1.18 & 1.04 \\
\hline 190 & 1.86 & 1.29 & 1.86 & 1.27 \\
\hline 195 & 2.74 & 1.44 & 2.64 & 1.48 \\
\hline 200 & 3.07 & 1.66 & 2.97 & 1.67 \\
\hline
\end{tabular}

Intersections of piecewise linear interpolations of the observed and expected rate limits with the $\mathrm{SM}=1$ line are used to quote ranges of Higgs boson masses that are excluded and that are expected to be excluded. The regions of Higgs boson masses excluded at the $95 \%$ C.L. are $90<$ $m_{H}<109 \mathrm{GeV} / c^{2}$ and $149<m_{H}<182 \mathrm{GeV} / c^{2}$. The expected exclusion regions are $90<m_{H}<120 \mathrm{GeV} / c^{2}$ and $140<m_{H}<184 \mathrm{GeV} / c^{2}$.

The observed excess for $m_{H}$ from 115 to $140 \mathrm{GeV} / c^{2}$ is driven by an excess of data events with respect to the background predictions in the most sensitive bins of the discriminant distributions, favoring the hypothesis that a signal is present. To characterize the compatibility of this excess with the signal-plus-background hypothesis, the best-fit rate cross section, $R^{\text {fit }}$, is computed using the Bayesian calculation, and shown in Fig. 6. The measured signal strength is within 1 s.d. of the expectation for a SM Higgs boson in the range $115<m_{H}<140 \mathrm{GeV} / c^{2}$, with maximal strength between $120 \mathrm{GeV} / c^{2}$ and $125 \mathrm{GeV} / c^{2}$. At $125 \mathrm{GeV} / c^{2}, R^{\mathrm{fit}}=1.44_{-0.47}^{+0.49}(\mathrm{stat})_{-0.31}^{+0.33} \times$ (syst) \pm 0.10 (theory).

The significance of the excess in the data over the background prediction is computed at each hypothesized Higgs boson mass by calculating the local $p$-value under the 


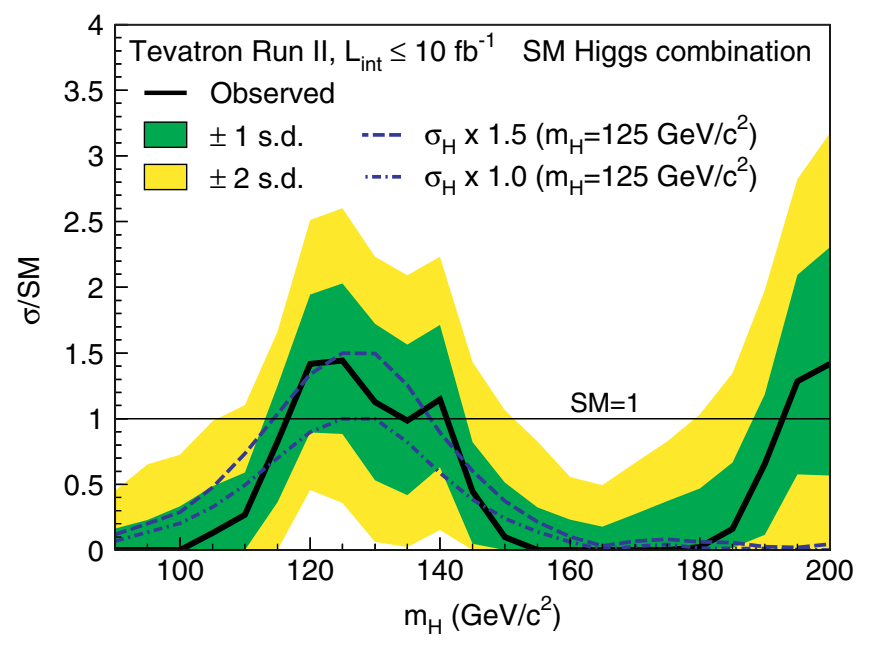

FIG. 6 (color online). The best-fit signal cross section expressed as a ratio to the SM cross section as a function of Higgs boson mass for all of CDF's and D0's SM Higgs boson searches in all decay modes combined. The dark- and lightshaded bands show the 1 and 2 s.d. uncertainty ranges on the fitted signal, respectively. Also shown with blue lines are the median fitted cross sections expected for a SM Higgs boson with $m_{H}=125 \mathrm{GeV} / c^{2}$ at signal strengths of 1.0 times (short-dashed) and 1.5 times (long-dashed) the SM prediction.

background-only hypothesis using $R_{\text {profile }}^{\mathrm{fit}}$, chosen a priori as the test statistic. These $p$-values express the probability to obtain the value of $R_{\text {profile }}^{\text {fit }}$ observed in the data or larger, assuming a signal is absent. These $p$-values are shown in Fig. 7 along with the expected $p$-values assuming a SM signal is present, separately for each value of $m_{H}$. The median expected $p$-values assuming the SM Higgs boson is present with $m_{H}=125 \mathrm{GeV} / c^{2}$ for signal strengths of 1.0 and 1.5 times the SM prediction are also shown. The median expected excess at $m_{H}=125 \mathrm{GeV} / c^{2}$ corresponds to 1.9 standard deviations assuming the SM Higgs boson is present at that mass. The observed local significance at $m_{H}=125 \mathrm{GeV} / c^{2}$ corresponds to 3.0 standard deviations. The maximum observed local significance is at $m_{H}=$ $120 \mathrm{GeV} / c^{2}$ and corresponds to 3.1 standard deviations. The fluctuations seen in the observed $p$-value as a function of the tested $m_{H}$ result from excesses seen in different search channels, as well as from point-to-point fluctuations due to the separate discriminants at each $m_{H}$, and are discussed in more detail below. The width of the dip in the observed $p$-values from 115 to $140 \mathrm{GeV} / c^{2}$ is consistent with the resolution of the combination of the $H \rightarrow b \bar{b}$ and $H \rightarrow$ $W^{+} W^{-}$channels, as illustrated by the injected signal curves in Fig. 7. The effective resolution of this search comes from two independent sources of information. The reconstructed candidate masses help constrain $m_{H}$, but more importantly, the expected cross sections times the relevant branching ratios for the $H \rightarrow b \bar{b}$ and $H \rightarrow W^{+} W^{-}$channels are strong functions of $m_{H}$ in the SM. The observed excess in the $H \rightarrow b \bar{b}$ channels coupled with the slight excess in

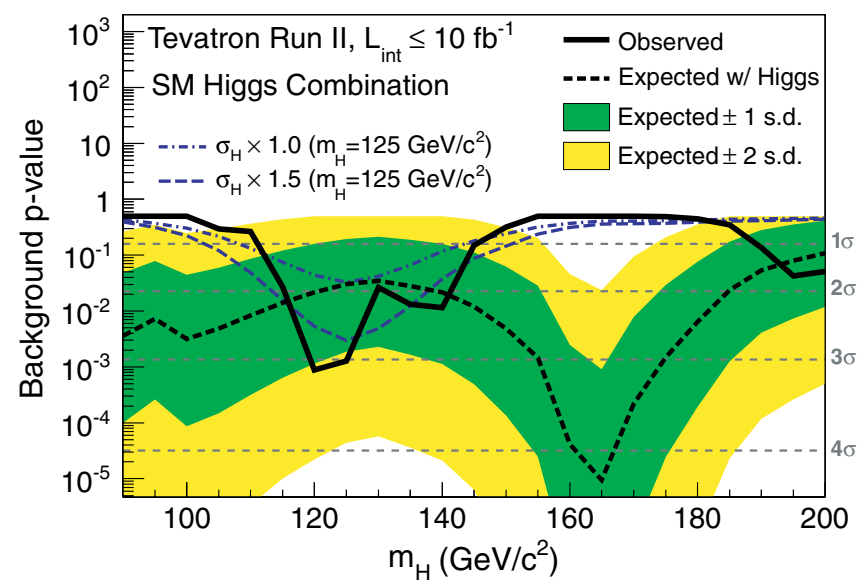

FIG. 7 (color online). The solid black line shows the background $p$-value as a function of $m_{H}$ for all of CDF's and D0's SM Higgs boson searches in all decay modes combined. The dotted black line shows the median expected values assuming a SM signal is present, evaluated separately at each $m_{H}$. The associated dark- and lightshaded bands indicate the 1 and 2 s.d. fluctuations of possible experimental outcomes under this scenario. The blue lines show the median expected $p$-values assuming the SM Higgs boson is present with $m_{H}=125 \mathrm{GeV} / c^{2}$ at signal strengths of 1.0 times (short-dashed) and 1.5 times (long-dashed) the SM prediction.

the $H \rightarrow W^{+} W^{-}$channels determines the shape of the observed $p$-value as a function of $m_{H}$.

Figure 8 shows the quantity $\mathrm{CL}_{\mathrm{s}+\mathrm{b}}$, corresponding to the $p$-value for the signal-plus-background hypothesis. The observed value, along with the expected $p$-values

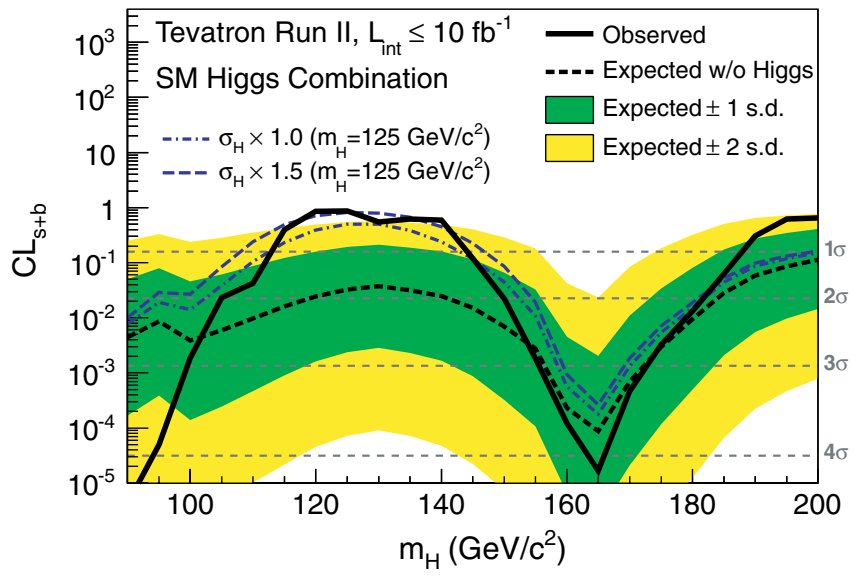

FIG. 8 (color online). The solid black line shows the signalplus-background $p$-value as a function of $m_{H}$ for all of CDF's and D0's SM Higgs boson searches in all decay modes combined. The dotted black line shows the median expected values assuming no SM signal is present, evaluated separately at each $m_{H}$. The associated dark- and light-shaded bands indicate the 1 and 2 s.d. fluctuations of possible experimental outcomes under this scenario. The blue lines show the median expected $p$-values assuming the SM Higgs boson is present with $m_{H}=$ $125 \mathrm{GeV} / c^{2}$ at signal strengths of 1.0 times (short-dashed) and 1.5 times (long-dashed) the SM prediction. 


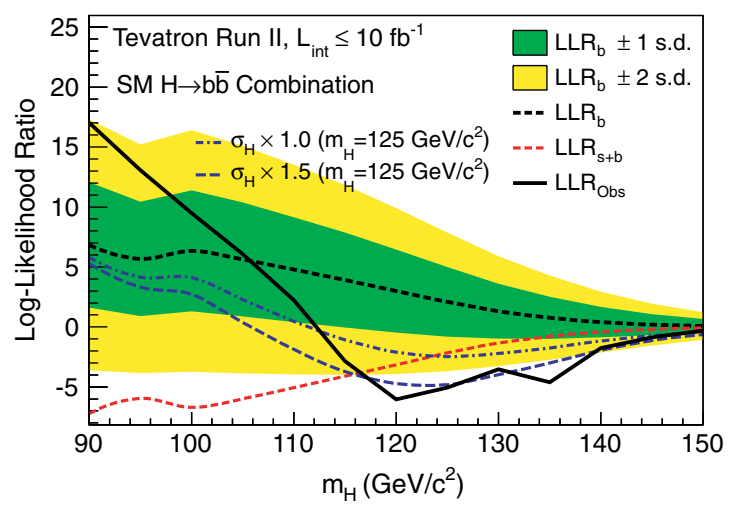

FIG. 9 (color online). LLR as a function of Higgs boson mass from the combination of CDF's and D0's SM Higgs boson searches focusing on the $H \rightarrow b \bar{b}$ decay mode. The solid line shows the observed LLR values, the dark short-dashed line shows the median expectation assuming no Higgs boson signal is present, and the dark- and light-shaded bands correspond, respectively, to the regions encompassing 1 and 2 s.d. fluctuations around the background-only expectation. The red long-dashed line shows the median expectation assuming a SM Higgs boson signal is present at each value of $m_{H}$ in turn. The blue lines show the median expected LLR assuming the SM Higgs boson is present at $m_{H}=125 \mathrm{GeV} / c^{2}$ with signal strengths of 1.0 times (short-dashed) and 1.5 times (long-dashed) the SM prediction.

assuming a signal is absent, is shown separately for each value of $m_{H}$. The median expected $p$-values assuming the SM Higgs boson is present with $m_{H}=125 \mathrm{GeV} / c^{2}$ for signal strengths of 1.0 and 1.5 times the SM prediction are also shown. In the mass region from 115 to $140 \mathrm{GeV} / c^{2}$ the observed values above $50 \%$ indicate a high level of consistency with the signal-plus-background hypothesis.

We also separate CDF's and D0's searches into combinations focusing on the $H \rightarrow b \bar{b}, H \rightarrow W^{+} W^{-}, H \rightarrow \gamma \gamma$, and $H \rightarrow \tau^{+} \tau^{-}$decay modes, and these are discussed in the following sections.

\section{C. $\boldsymbol{H} \rightarrow \boldsymbol{b} \bar{b}$ decay mode}

Below $130 \mathrm{GeV} / c^{2}$, the $H \rightarrow b \bar{b}$ searches contribute the majority of the search sensitivity. The $W H \rightarrow \ell \nu b \bar{b}, Z H \rightarrow$ $\nu \bar{\nu} b \bar{b}$, and $Z H \rightarrow \ell^{+} \ell^{-} b \bar{b}$ channels from both experiments are included in this subcombination. Two of the six contributing channels were updated for this subcombination compared with that reported in Ref. [12]. The CDF $Z H \rightarrow \nu \bar{\nu} b \bar{b}$ [74] analysis was updated to use a more powerful MVA $b$-tagging algorithm [62] along with changes to the kinematic selections. The assignment of correlated systematic uncertainties between channels was updated in the D0 $W H \rightarrow \ell \nu b \bar{b}$ analysis [51]. The observed LLR distribution is shown in Fig. 9, along with its expected values under the background-only and signal-plus-background hypotheses. The hypotheses that a SM Higgs boson is present with $m_{H}=125 \mathrm{GeV} / c^{2}$ for signal strengths of 1.0 and 1.5 times the SM prediction are also given. The LLR values as a function of Higgs boson mass are listed in Table V.

We multiply the best-fit rate cross section, $R^{\mathrm{fit}}$, for this subcombination by the SM prediction for the associatedproduction cross section times the decay branching ratio $\left(\sigma_{W H}+\sigma_{Z H}\right) \times \mathcal{B}(H \rightarrow b \bar{b})$, to obtain the observed value for this quantity. We show the fitted $\left(\sigma_{W H}+\sigma_{Z H}\right) \times$ $\mathcal{B}(H \rightarrow b \bar{b})$ as a function of $m_{H}$, along with the SM prediction, in Fig. 10. The figure also shows the expected cross section fits for each $m_{H}$, assuming that the SM Higgs boson with $m_{H}=125 \mathrm{GeV} / c^{2}$ is present, both at the rate predicted by the SM and also at a multiple of 1.5 times that of the SM. The best-fit rate corresponds to $\left(\sigma_{W H}+\sigma_{Z H}\right) \times$ $\mathcal{B}(H \rightarrow b \bar{b})=0.19_{-0.09}^{+0.08}$ (stat + syst) pb. The shift in this result compared with the value of $0.23 \pm 0.09$ (stat + syst) $\mathrm{pb}$ obtained previously [12] is due to the updated $Z H \rightarrow \nu \bar{\nu} b \bar{b}$ analysis from CDF [43,74] and corresponds to a change in the central value of 0.5 times the total uncertainty. For $m_{H}=125 \mathrm{GeV} / c^{2}$, the $\mathrm{SM}$ predicts $\left(\sigma_{W H}+\sigma_{Z H}\right) \times \mathcal{B}(H \rightarrow b \bar{b})=0.12 \pm 0.01 \mathrm{pb}$.

TABLE V. LLR values obtained from the combination of CDF's and D0's Higgs boson search channels focusing on the $H \rightarrow b \bar{b}$ decay mode using the $\mathrm{CL}_{\mathrm{s}}$ method.

\begin{tabular}{lccccccc}
\hline \hline$m_{H}\left(\mathrm{GeV} / c^{2}\right)$ & $\mathrm{LLR}_{\text {obs }}$ & $\mathrm{LLR}_{s+b}$ & $\mathrm{LLR}_{b}^{-2 \sigma}$ & $\mathrm{LLR}_{b}^{-1 \sigma}$ & $\mathrm{LLR}_{b}$ & $\mathrm{LLR}_{b}^{+1 \sigma}$ & $\mathrm{LLR}_{b}^{+2 \sigma}$ \\
\hline 90 & 17.02 & -7.24 & 17.31 & 12.08 & 6.84 & 1.61 & -3.62 \\
95 & 13.07 & -5.96 & 15.21 & 10.44 & 5.68 & 0.91 & -3.85 \\
100 & 9.50 & -6.71 & 16.41 & 11.38 & 6.34 & 1.30 & -3.73 \\
105 & 6.09 & -6.00 & 15.12 & 10.38 & 5.63 & 0.88 & -3.86 \\
110 & 2.21 & -5.06 & 13.52 & 9.15 & 4.78 & 0.41 & -3.97 \\
115 & -2.84 & -4.12 & 11.83 & 7.87 & 3.91 & -0.04 & -4.00 \\
120 & -6.05 & -3.16 & 9.91 & 6.45 & 2.99 & -0.47 & -3.93 \\
125 & -5.05 & -2.16 & 7.88 & 4.99 & 2.09 & -0.80 & -3.69 \\
130 & -3.53 & -1.34 & 5.89 & 3.60 & 1.31 & -0.98 & -3.27 \\
135 & -4.60 & -0.80 & 4.28 & 2.52 & 0.77 & -0.98 & -2.74 \\
140 & -1.77 & -0.41 & 2.93 & 1.67 & 0.40 & -0.87 & -2.13 \\
145 & -0.86 & -0.20 & 1.95 & 1.07 & 0.19 & -0.68 & -1.56 \\
150 & -0.31 & -0.08 & 1.23 & 0.66 & 0.08 & -0.49 & -1.07 \\
\hline \hline
\end{tabular}


HIGGS BOSON STUDIES AT THE TEVATRON

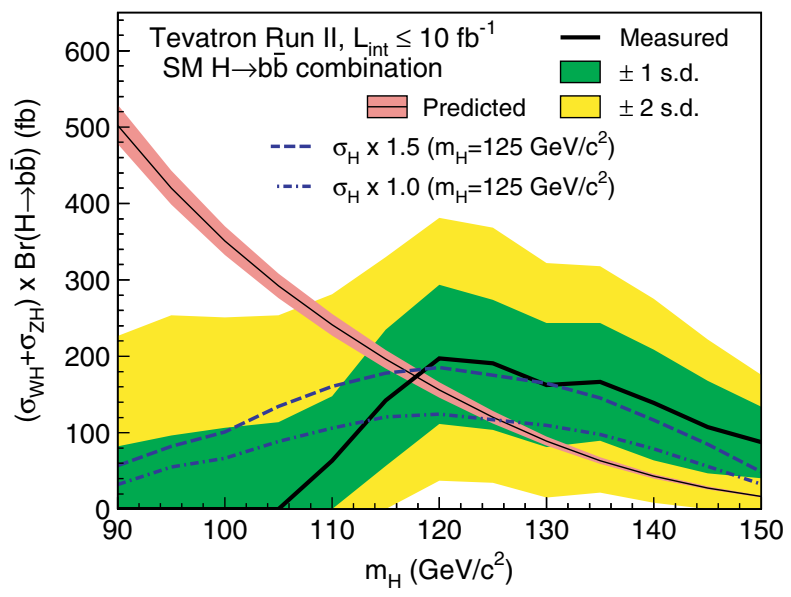

FIG. 10 (color online). The best-fit signal cross section times branching ratio $\left(\sigma_{W H}+\sigma_{Z H}\right) \times \mathcal{B}(H \rightarrow b \bar{b})$ as a function of Higgs boson mass from the combination of CDF's and D0's SM Higgs boson searches focusing on the $H \rightarrow b \bar{b}$ decay mode. The dark- and light-shaded bands show the 1 and 2 s.d. uncertainty ranges on the fitted signal, respectively. Also shown with blue lines are the median fitted cross sections expected for a SM Higgs boson with $m_{H}=125 \mathrm{GeV} / c^{2}$ at signal strengths of 1.0 times (short-dashed) and 1.5 times (long-dashed) the SM prediction. The SM prediction is shown as the smooth, falling curve where the narrow band indicates the theoretical uncertainty.

\section{D. $\boldsymbol{H} \rightarrow \boldsymbol{W}^{+} \boldsymbol{W}^{-}$decay mode}

Above $130 \mathrm{GeV} / c^{2}$, the $H \rightarrow W^{+} W^{-}$channels contribute the majority of the search sensitivity. We combine all $H \rightarrow W^{+} W^{-}$searches from CDF and D0, incorporating potential signal contributions from gluon-gluon fusion, $W H, \quad Z H$, and vector boson fusion production. Approximately $75 \%$ of the signal comes from the gluongluon fusion process, $20 \%$ from associated production, and $5 \%$ from the VBF process. The LLR distributions are shown in Fig. 11 and the values as a function of Higgs boson mass are listed in Table VI. The data present a 1 to 2 s.d. excess in the region from 115 to $140 \mathrm{GeV} / c^{2}$ where there is some separation between the two hypotheses. An excess is also seen in the searches for Higgs bosons with mass $m_{H}>195 \mathrm{GeV} / c^{2}$, as mentioned in Sec. VIII B, but the sensitivity to the SM Higgs boson is not as large at these masses as it is at lower masses. Figure 12 shows the best-fit cross section for the combined $H \rightarrow W^{+} W^{-}$ searches, normalized to the SM prediction, as a function of $m_{H}$, along with the expectations assuming the SM Higgs boson is present at $m_{H}=125 \mathrm{GeV} / c^{2}$ for signal strengths of 1.0 and 1.5 times the SM prediction.

\section{E. $H \rightarrow \gamma \gamma$ decay mode}

We also separately combine CDF's and D0's searches focusing on the $H \rightarrow \gamma \gamma$ decay mode and display the resulting upper limits on the production cross section times the decay branching ratio normalized to the SM prediction in Fig. 13. An excess of approximately 2 s.d. is seen in
PHYSICAL REVIEW D 88, 052014 (2013)

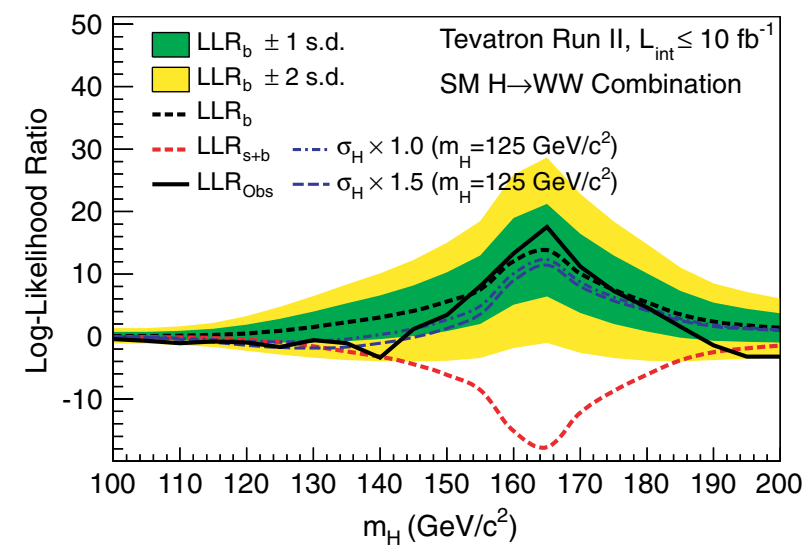

FIG. 11 (color online). LLR as a function of Higgs boson mass from the combination of CDF's and D0's SM Higgs boson searches focusing on the $H \rightarrow W^{+} W^{-}$decay mode. The solid line shows the observed LLR values, the dark short-dashed line shows the median expectation assuming no Higgs boson signal is present, and the dark- and light-shaded bands correspond, respectively, to the regions encompassing 1 and 2 s.d. fluctuations around the background-only expectation. The red longdashed line shows the median expectation assuming a SM Higgs boson signal is present at each value of $m_{H}$ in turn. The blue lines show the median expected LLR assuming the SM Higgs boson is present at $m_{H}=125 \mathrm{GeV} / c^{2}$ with signal strengths of 1.0 times (short-dashed) and 1.5 times (longdashed) the SM prediction.

these searches at $m_{H}=125 \mathrm{GeV} / c^{2}$, but its contributions to the fully combined SM cross section and limit are small due to the low expected signal yield in this channel. However, the observed excess in the $H \rightarrow \gamma \gamma$ search channel has a visible impact on Higgs boson coupling constraints as described in Sec. VIII G.

\section{F. $H \rightarrow \tau \tau$ decay mode}

We also separately combine CDF's and D0's searches focusing on the $H \rightarrow \tau^{+} \tau^{-}$decay mode and display the resulting upper limits on the production cross section times the decay branching ratio normalized to the SM prediction in Fig. 14.

\section{G. Compatibility of the excess with the SM Higgs boson hypothesis}

The best-fit rate parameters, $R^{\text {fit }}$, for the full combination of all channels and the combinations of channels focusing on the $H \rightarrow W^{+} W^{-}, H \rightarrow b \bar{b}, H \rightarrow \gamma \gamma$, and $H \rightarrow \tau^{+} \tau^{-}$decay modes [75] are listed in Table VII as a function of Higgs boson mass over the range $115<m_{H}<140 \mathrm{GeV} / c^{2}$, where the combined result has sensitivity to a signal and a clear excess exists. For $m_{H}=125 \mathrm{GeV} / c^{2}$, we obtain $R^{\mathrm{fit}}=1.44_{-0.56}^{+0.59}$ using all decay modes.

Figure 15 shows the contribution of the four combinations for the different decay modes to the best-fit signal cross 
TABLE VI. LLR values obtained from the combination of CDF's and D0's Higgs boson search channels focusing on the $H \rightarrow W^{+} W^{-}$decay mode using the $\mathrm{CL}_{\mathrm{s}}$ method.

\begin{tabular}{lrrrrrrr}
\hline \hline$m_{H}\left(\mathrm{GeV} / c^{2}\right)$ & $\mathrm{LLR}_{\mathrm{obs}}$ & $\mathrm{LLR}_{s+b}$ & $\mathrm{LLR}_{b}^{-2 \sigma}$ & $\mathrm{LLR}_{b}^{-1 \sigma}$ & $\mathrm{LLR}_{b}$ & $\mathrm{LLR}_{b}^{+1 \sigma}$ & $\mathrm{LLR}_{b}^{+2 \sigma}$ \\
\hline 100 & -0.42 & -0.10 & 1.36 & 0.73 & 0.10 & -0.53 & -1.16 \\
105 & -0.72 & -0.10 & 1.34 & 0.72 & 0.10 & -0.53 & -1.15 \\
110 & -1.07 & -0.14 & 1.62 & 0.88 & 0.14 & -0.60 & -1.34 \\
115 & -0.80 & -0.24 & 2.18 & 1.21 & 0.24 & -0.74 & -1.71 \\
120 & -0.98 & -0.49 & 3.26 & 1.87 & 0.48 & -0.91 & -2.30 \\
125 & -1.69 & -0.94 & 4.73 & 2.82 & 0.91 & -1.00 & -2.91 \\
130 & -0.59 & -1.57 & 6.44 & 3.98 & 1.52 & -0.95 & -3.41 \\
135 & -1.11 & -2.40 & 8.36 & 5.33 & 2.30 & -0.73 & -3.77 \\
140 & -3.38 & -3.24 & 10.09 & 6.58 & 3.08 & -0.43 & -3.94 \\
145 & 1.19 & -4.42 & 12.23 & 8.17 & 4.12 & 0.06 & -4.00 \\
150 & 3.43 & -6.13 & 15.01 & 10.29 & 5.57 & 0.85 & -3.87 \\
155 & 8.05 & -8.59 & 18.45 & 12.97 & 7.50 & 2.02 & -3.46 \\
160 & 13.27 & -15.15 & 25.92 & 18.98 & 12.04 & 5.10 & -1.84 \\
165 & 17.55 & -17.75 & 28.69 & 21.25 & 13.82 & 6.38 & -1.05 \\
170 & 11.19 & -12.21 & 22.80 & 16.45 & 10.09 & 3.74 & -2.61 \\
175 & 7.28 & -8.72 & 18.44 & 12.96 & 7.49 & 2.02 & -3.46 \\
180 & 4.63 & -6.12 & 14.80 & 10.13 & 5.46 & 0.78 & -3.89 \\
185 & 1.56 & -3.83 & 11.05 & 7.29 & 3.53 & -0.23 & -3.99 \\
190 & -1.39 & -2.50 & 8.51 & 5.44 & 2.36 & -0.71 & -3.79 \\
195 & -3.24 & -1.88 & 7.12 & 4.45 & 1.78 & -0.89 & -3.56 \\
200 & -3.23 & -1.45 & 6.08 & 3.73 & 1.38 & -0.97 & -3.32 \\
\hline \hline
\end{tabular}

section for $m_{H}=125 \mathrm{GeV} / c^{2}$. The results are consistent with each other, with the full combination, and with the production of the SM Higgs boson at that mass. Figure 16 shows the posterior probability densities obtained for the

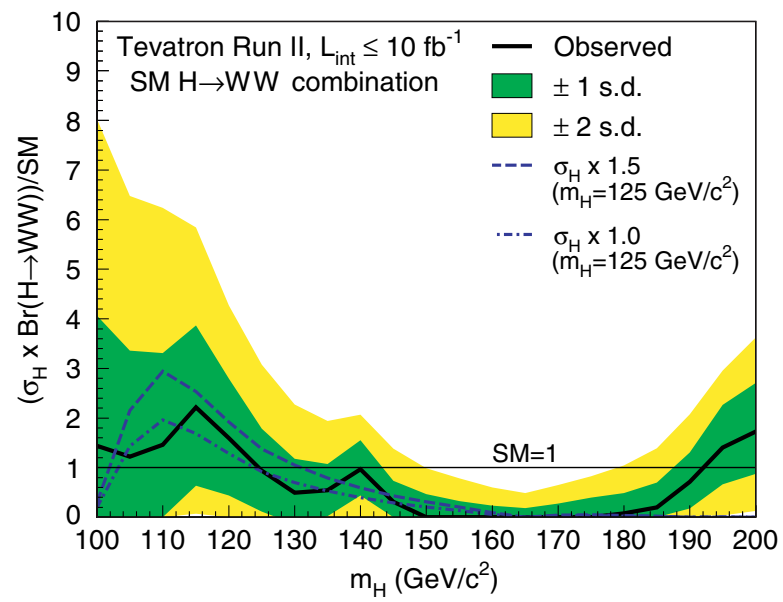

FIG. 12 (color online). The best-fit signal cross section expressed as a ratio to the SM cross section as a function of Higgs boson mass from the combination of CDF's and D0's SM Higgs boson searches focusing on the $H \rightarrow W^{+} W^{-}$decay mode. The dark- and light-shaded bands show the 1 and 2 s.d. uncertainty ranges on the fitted signal, respectively. Also shown with blue lines are the median fitted cross sections expected for a SM Higgs boson with $m_{H}=125 \mathrm{GeV} / c^{2}$ at signal strengths of 1.0 times (shortdashed) and 1.5 times (long-dashed) the SM prediction. cross-section scale factors from the $H \rightarrow b \bar{b}, H \rightarrow W^{+} W^{-}$, $H \rightarrow \gamma \gamma$, and $H \rightarrow \tau^{+} \tau^{-}$combinations.

The Higgs boson is expected to couple more strongly to more massive particles than to less massive ones, and thus

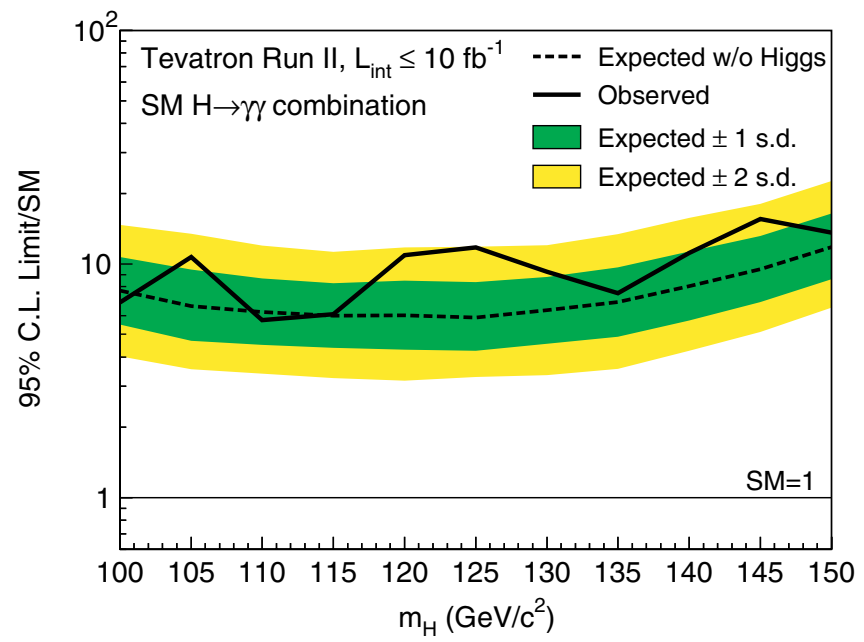

FIG. 13 (color online). Observed and median expected (for the background-only hypothesis) $95 \%$ C.L. Bayesian upper production limits expressed as multiples of the SM cross section as a function of Higgs boson mass from the combination of CDF's and D0's SM Higgs boson searches focusing on the $H \rightarrow \gamma \gamma$ decay mode. The dark- and light-shaded bands indicate, respectively, the 1 and 2 s.d. probability regions in which the limits are expected to fluctuate in the absence of signal. 
HIGGS BOSON STUDIES AT THE TEVATRON

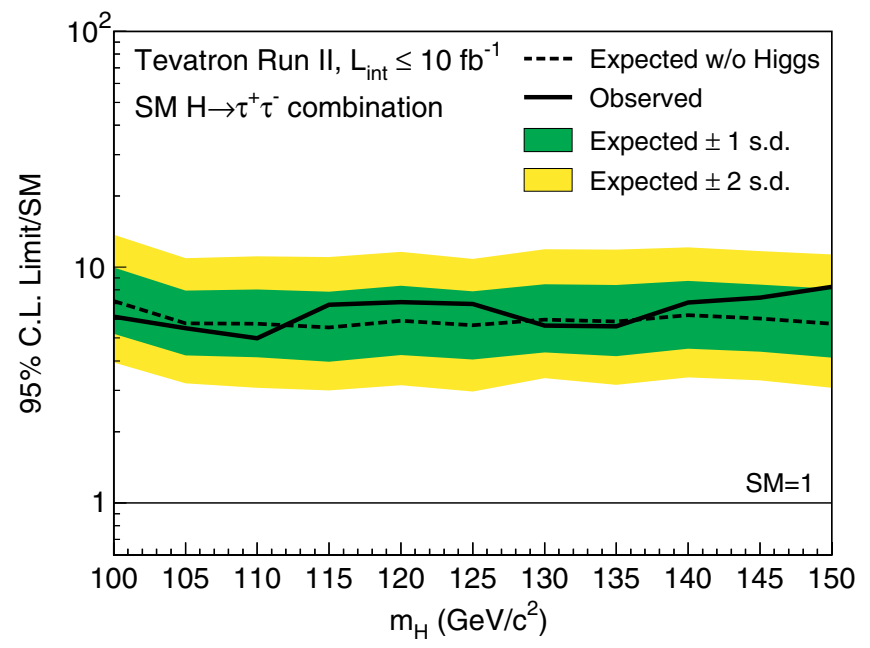

FIG. 14 (color online). Observed and median expected (for the background-only hypothesis) $95 \%$ C.L. Bayesian upper production limits expressed as multiples of the SM cross section as a function of Higgs boson mass from the combination of CDF's and D0's SM Higgs boson searches focusing on the $H \rightarrow \tau^{+} \tau^{-}$ decay mode. The dark- and light-shaded bands indicate, respectively, the 1 and 2 s.d. probability regions in which the limits are expected to fluctuate in the absence of signal.

may provide sensitivity to non-SM particles whose interactions become more relevant at higher energies. It is important therefore to study in detail the properties of the new particle. The channel-by-channel values of $R=(\sigma \times \mathcal{B}) /$ SM provide useful constraints on the possible couplings of the particle [76], but their interpretation is ambiguous because signal contributions from multiple sources are simultaneously accepted by each subchannel. For example, the $Z H \rightarrow \nu \bar{\nu} b \bar{b}$ channels have sensitivity to both the $W H$ and $Z H$ production modes, and the $H \rightarrow W^{+} W^{-}$searches are sensitive to gluon-gluon fusion, $W H, Z H$, and VBF in different mixtures within independent subchannels characterized by the number of reconstructed jets.

Most of the searches conducted at the Tevatron are sensitive to the product of fermion and boson coupling strengths. In the $V H \rightarrow V b \bar{b}$ searches, the production depends on the coupling of the Higgs boson to the weak
PHYSICAL REVIEW D 88, 052014 (2013)

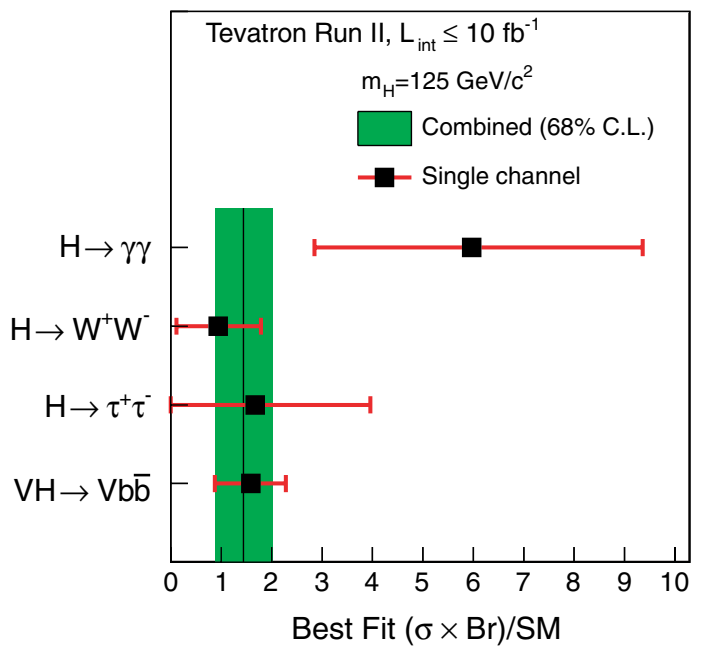

FIG. 15 (color online). Best-fit values of $R=(\sigma \times \mathcal{B}) / \mathrm{SM}$ using the Bayesian method for the combinations of CDF's and D0's Higgs boson search channels focusing on the $H \rightarrow W^{+} W^{-}$, $H \rightarrow b \bar{b}, H \rightarrow \gamma \gamma$, and $H \rightarrow \tau^{+} \tau^{-}$decay modes for a Higgs boson mass of $125 \mathrm{GeV} / c^{2}$. The shaded band corresponds to the 1 s.d. uncertainty on the best-fit value of $R$ for all SM Higgs boson decay modes combined.

vector bosons, while the decay is to fermions. In the $g g \rightarrow$ $H \rightarrow W^{+} W^{-}$searches, the production is dominated by the Higgs boson couplings to fermions via the quark loop processes, but the decay is to bosons. A large enhancement of the Higgs boson's couplings to fermions can thus be masked by a small coupling to bosons, and vice versa, as shown in Fig. 2 of Ref. [76]. However, other less-sensitive channels included in this combination provide additional constraints. The same-sign dilepton searches, the trilepton searches, and some of the searches with tau leptons as decay products of $W$ bosons are primarily sensitive to $V H \rightarrow V W^{+} W^{-}$, an entirely bosonic process, although their results are customarily reported in combination with the other $H \rightarrow W^{+} W^{-}$searches. The searches for $t \bar{t} H \rightarrow$ $t \bar{t} b \bar{b}$ provide constraints on the fermion couplings with minimal masking from the bosonic couplings.

We follow the notation of Ref. [77] and introduce multiplicative scaling factors for the coupling of the Higgs boson

TABLE VII. Best-fit values of $R=(\sigma \times \mathcal{B}) /$ SM using the Bayesian method for all SM Higgs boson decay modes combined and the combinations of CDF's and D0's Higgs boson search channels focusing on the $H \rightarrow W^{+} W^{-}, H \rightarrow b \bar{b}, H \rightarrow \gamma \gamma$, and $H \rightarrow \tau^{+} \tau^{-}$ decay modes as a function of Higgs boson mass over the range $115<m_{H}<140 \mathrm{GeV} / c^{2}$. The quoted uncertainties bound the smallest interval containing $68 \%$ of the integral of the posterior probability density.

\begin{tabular}{|c|c|c|c|c|c|c|}
\hline$m_{H}\left(\mathrm{GeV} / c^{2}\right)$ & 115 & 120 & 125 & 130 & 135 & 140 \\
\hline$R_{\mathrm{fit}}(\mathrm{SM})$ & $0.82_{-0.46}^{+0.43}$ & $1.42_{-0.52}^{+0.53}$ & $1.44_{-0.56}^{+0.59}$ & $1.13_{-0.60}^{+0.60}$ & $0.99_{-0.57}^{+0.58}$ & $1.15_{-0.52}^{+0.57}$ \\
\hline$R_{\mathrm{fit}}\left(H \rightarrow W^{+} W^{-}\right)$ & $2.22_{-1.59}^{+1.65}$ & $1.59_{-1.15}^{+1.20}$ & $0.94_{-0.83}^{+0.85}$ & $0.49_{-0.63}^{+0.69}$ & $0.54_{-0.52}^{+0.53}$ & $0.97_{-0.53}^{+0.58}$ \\
\hline$R_{\mathrm{fit}}(H \rightarrow b \bar{b})$ & $0.72_{-0.44}^{+0.47}$ & $1.26_{-0.55}^{+0.62}$ & $1.59_{-0.72}^{+0.69}$ & $1.82_{-0.91}^{+0.91}$ & $2.62_{-1.21}^{+1.22}$ & $3.23_{-1.74}^{+1.61}$ \\
\hline$R_{\mathrm{fit}}(H \rightarrow \gamma \gamma)$ & $0.65_{-0.54}^{+2.66}$ & $5.34_{-2.76}^{+3.20}$ & $5.97_{-3.12}^{+3.39}$ & $3.17_{-2.81}^{+2.69}$ & $0.00_{-0.00}^{+4.04}$ & $3.31_{-3.13}^{+3.30}$ \\
\hline$R_{\mathrm{fit}}\left(H \rightarrow \tau^{+} \tau^{-}\right)$ & $1.70_{-1.70}^{+2.20}$ & $2.00_{-1.90}^{+2.22}$ & $1.68_{-1.68}^{+2.28}$ & $0.00_{-0.00}^{+2.88}$ & $0.00_{-0.00}^{+2.83}$ & $1.25_{-1.15}^{+2.62}$ \\
\hline
\end{tabular}



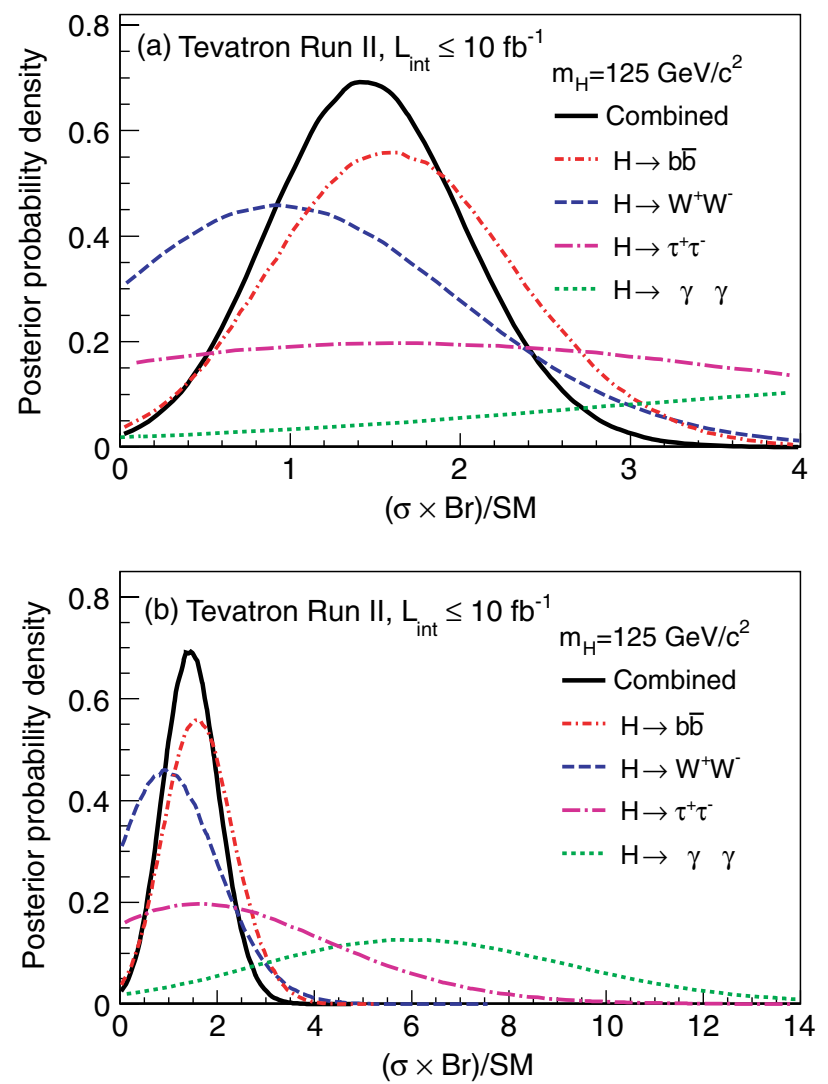

FIG. 16 (color online). (a) Posterior probability densities for $R=(\sigma \times \mathcal{B}) / \mathrm{SM}$ using the Bayesian method from the combinations of CDF's and D0's Higgs boson search channels focusing on the $H \rightarrow W^{+} W^{-}, H \rightarrow b \bar{b}, H \rightarrow \gamma \gamma$, and $H \rightarrow \tau^{+} \tau^{-}$decay modes and for all SM Higgs boson decay modes combined. The same curves are shown on an expanded scale in (b).

to fermions $\left(\kappa_{f}\right)$ and either to $W$ bosons $\left(\kappa_{W}\right)$ and $Z$ bosons $\left(\kappa_{Z}\right)$ or more generically to vector bosons $\left(\kappa_{V}\right)$. We then search for deviations from the expected SM values of $\kappa_{i}=1$.

The first test assumes $m_{H}=125 \mathrm{GeV} / c^{2}$, based on the ATLAS and CMS observations, and fits for the $H \rightarrow$ $W^{+} W^{-}$coupling, holding all other couplings fixed to their SM values. This test corresponds to holding the values of $\kappa_{Z}=\kappa_{f}=1$, while varying $\kappa_{W}$. At each value of $\kappa_{W}$, we recompute the predicted cross sections and decay branching ratios, as described in Ref. [77]. We assume a uniform prior density in $\kappa_{W}$, and show the posterior probability distribution in Fig. 17. A negative sign of $\kappa_{W}$ is preferred by the Tevatron data due to the excess seen in the $H \rightarrow \gamma \gamma$ searches. In the SM, this process proceeds at lowest order via a $W$-boson loop or a quark loop (dominated by the top quark), with destructive interference between the two contributions [78], as given by $\Gamma(H \rightarrow \gamma \gamma)=\Gamma(H \rightarrow$ $\gamma \gamma)_{\mathrm{SM}} \times\left|1.28 \kappa_{V}-0.28 \kappa_{f}\right|^{2}$. If the sign of the $H \rightarrow$ $W^{+} W^{-}$coupling is negative, then this interference becomes constructive, allowing for a larger prediction of the yield. We obtain a best-fit value of $\kappa_{W}=-1.27$. Our procedure for finding the smallest set of intervals

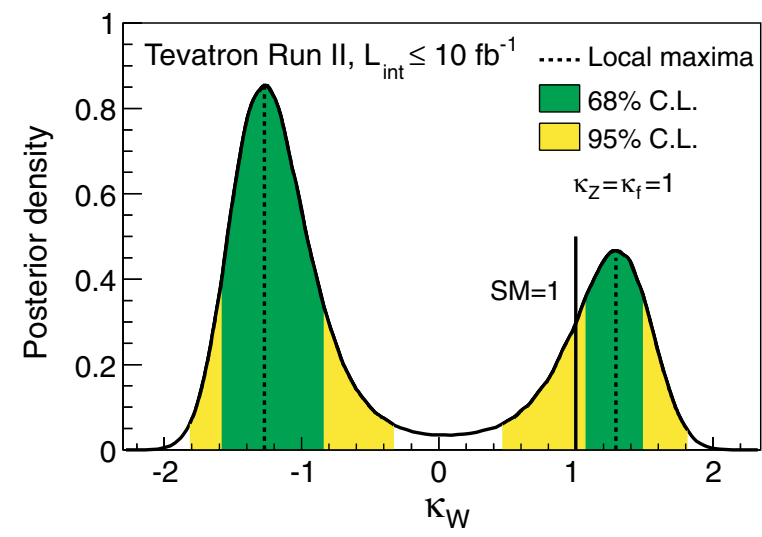

FIG. 17 (color online). Posterior probability density for $\kappa_{W}$ from the combination of Tevatron searches for a SM-like Higgs boson with $m_{H}=125 \mathrm{GeV} / c^{2}$. The couplings of the Higgs boson to fermions and to the $Z$ boson are assumed to be as predicted by the SM. The values that maximize the local posterior probability densities are shown with dashed lines, and the $68 \%$ and $95 \%$ C.L. intervals are indicated with the dark- and light-shaded regions, respectively. The predicted SM value of $\kappa_{W}$ is indicated by the solid vertical line.

that contain $68 \%$ of the integral of the posterior results in two intervals, $-1.56<\kappa_{W}<-0.81$ and $1.04<\kappa_{W}<$ 1.51. We perform a similar test for $\kappa_{Z}$, assuming $\kappa_{W}=$ $\kappa_{f}=1$. The resulting posterior probability density is shown in Fig. 18. The Higgs boson searches at the Tevatron are sensitive almost exclusively to the square of $\kappa_{Z}$, and thus the posterior density is nearly symmetric in positive and negative couplings. The best-fit values are $\kappa_{Z}=1.05_{-0.55}^{+0.45}$ and $\kappa_{Z}=-1.05_{-0.45}^{+0.55}$. Finally, we perform

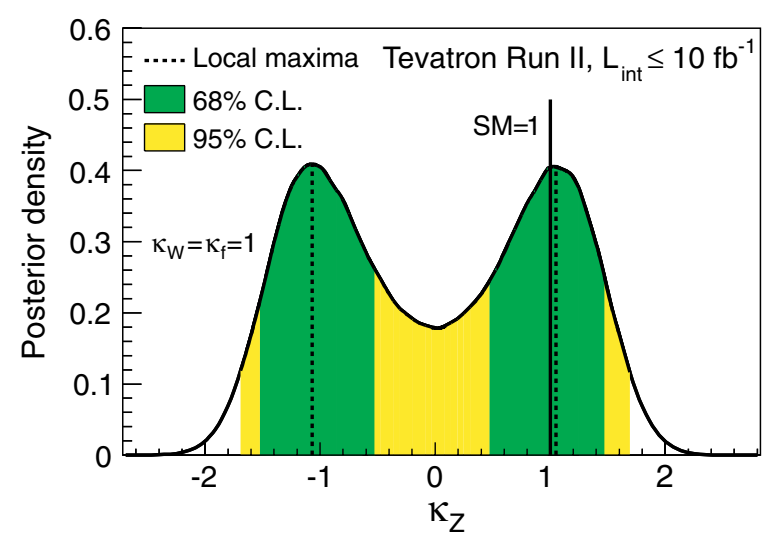

FIG. 18 (color online). Posterior probability density for $\kappa_{Z}$ from the combination of Tevatron searches for a SM-like Higgs boson with $m_{H}=125 \mathrm{GeV} / c^{2}$. The couplings of the Higgs boson to fermions and to the $W^{ \pm}$boson are assumed to be as predicted by the SM. The values that maximize the local posterior probability densities are shown with dashed lines, and the $68 \%$ and $95 \%$ C.L. intervals are indicated with the dark- and light-shaded regions, respectively. The predicted SM value of $\kappa_{Z}$ is indicated by the solid vertical line. 
a similar test for $\kappa_{f}$, the common scale factor on the Higgs boson couplings to fermions, holding $\kappa_{W}=\kappa_{Z}=1$. The resulting posterior probability density is shown in Fig. 19. An asymmetry is seen in this distribution, due again to the outcome in the $H \rightarrow \gamma \gamma$ channels. We obtain a best-fit value of $\kappa_{f}=-2.64_{-1.30}^{+1.59}$. The large magnitude of the fitted value is due to the excesses seen in the $H \rightarrow b \bar{b}$ and $H \rightarrow \gamma \gamma$ searches.

We then allow both $\kappa_{W}$ and $\kappa_{Z}$ to vary independently, also allowing $\kappa_{f}$ to vary by integrating the likelihood function times a uniform prior in $\kappa_{f}$ over negative and positive values. The resulting areas in the $\left(\kappa_{W}, \kappa_{Z}\right)$ plane preferred by the Tevatron data are shown in Fig. 20. While we allow either coupling scale factor to be negative, only two quadrants are shown in Fig. 20 due to an overall sign ambiguity. The point $\left(\kappa_{W}, \kappa_{Z}\right)=(0,0)$ corresponds to no Higgs boson production or decay in the most sensitive search modes at the Tevatron and is excluded at more than $95 \%$ C.L. due to the Higgs-boson-like signal in the $H \rightarrow b \bar{b}$ and $H \rightarrow W^{+} W^{-}$channels. Our best-fit points are $\left(\kappa_{W}, \kappa_{Z}\right)=(1.25, \pm 0.90)$.

We study the ratio $\lambda_{W Z}=\kappa_{W} / \kappa_{Z}$ using the same posterior probability density that is used in Fig. 20. We choose a projection onto a one-dimensional variable that preserves the uniformity of the prior probability density in the twodimensional plane. This variable is the angle with respect to the $\kappa_{W}$ axis, $\theta_{W Z}=\tan ^{-1}\left(\kappa_{Z} / \kappa_{W}\right)=\tan ^{-1}\left(1 / \lambda_{W Z}\right)$. Figure 21 shows the one-dimensional posterior probability density in this variable. This function is symmetric for positive and negative $\theta_{W Z}$. We measure $\left|\theta_{W Z}\right|=$ $0.68_{-0.41}^{+0.21}$, which corresponds to $\lambda_{W Z}=1.24_{-0.42}^{+2.34}$.

Assuming that custodial symmetry [79] holds $\left(\lambda_{W Z}=1\right)$, we allow both $\kappa_{V}$ and $\kappa_{f}$ to vary, and show in Fig. 22

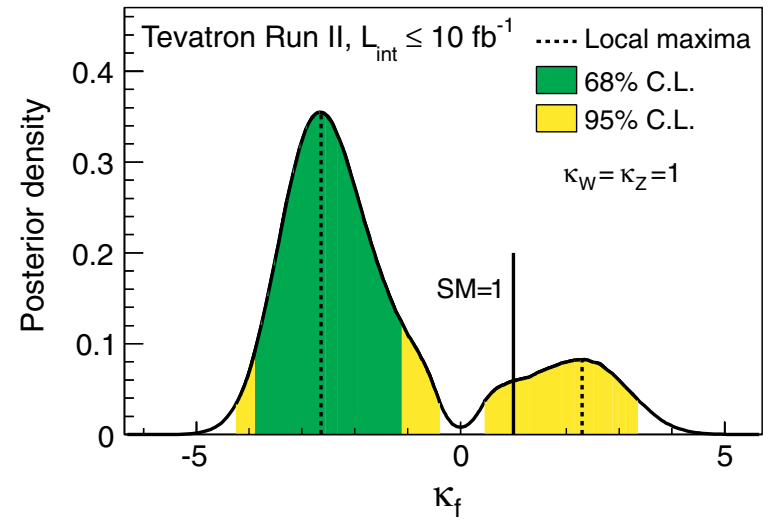

FIG. 19 (color online). Posterior probability density for $\kappa_{f}$ from the combination of Tevatron searches for a SM-like Higgs boson with $m_{H}=125 \mathrm{GeV} / c^{2}$. The couplings of the Higgs boson to the $W^{ \pm}$and $Z$ bosons are assumed to be as predicted by the SM. The values that maximize the local posterior probability densities are shown with dashed lines, and the $68 \%$ and $95 \%$ C.L. intervals are indicated with the dark- and light-shaded regions, respectively. The predicted SM value of $\kappa_{f}$ is indicated by the solid vertical line.

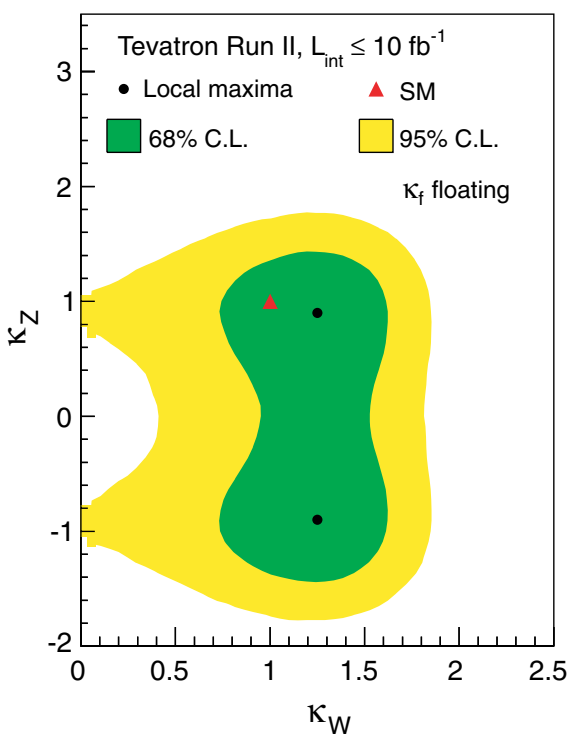

FIG. 20 (color online). Two-dimensional constraints in the $\left(\kappa_{W}, \kappa_{Z}\right)$ plane, for the combined Tevatron searches for a SM-like Higgs boson with mass $125 \mathrm{GeV} / c^{2}$ allowing $\kappa_{f}$ to float. The points that maximize the local posterior probability densities are marked with dots, and the $68 \%$ and $95 \%$ C.L. intervals are indicated with the dark- and light-shaded regions, respectively. The SM prediction for $\left(\kappa_{W}, \kappa_{Z}\right)$ is marked with a triangle.

the regions preferred at the $68 \%$ C.L. and the 95\% C.L. in the two-dimensional plane $\left(\kappa_{V}, \kappa_{f}\right)$. The asymmetry induced by the excesses in the $H \rightarrow \gamma \gamma$ searches is visible in this projection as well. The best-fit point is $\left(\kappa_{V}, \kappa_{f}\right)=$ $(1.05,-2.40)$, but a secondary maximum in the posterior density is seen at $\left(\kappa_{V}, \kappa_{f}\right)=(1.05,2.30)$, consistent with the SM expectation, given the large uncertainties. The integral of the posterior density in the $(+,+)$ quadrant is $26 \%$

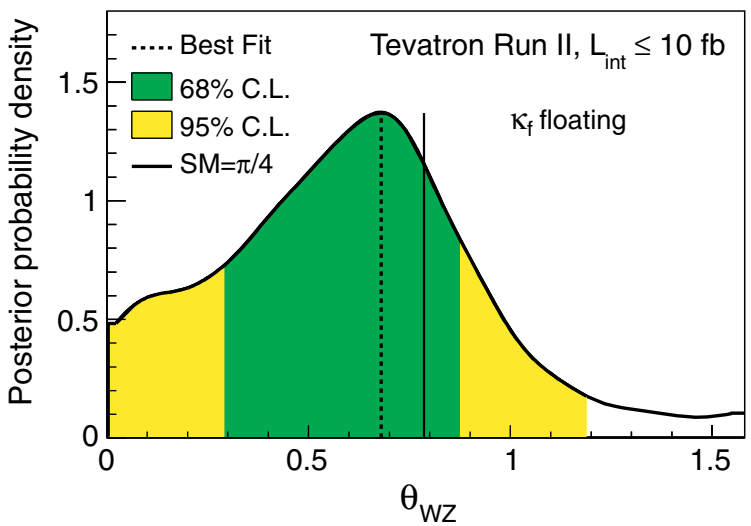

FIG. 21 (color online). Posterior probability density for $\theta_{W Z}=$ $\tan ^{-1}\left(\kappa_{Z} / \kappa_{W}\right)$, from the combination of Tevatron searches for a SM-like Higgs boson with $m_{H}=125 \mathrm{GeV} / c^{2}$ allowing $\kappa_{f}$ to float. The value that maximizes the posterior probability density is shown with a dashed vertical line, and the $68 \%$ and $95 \%$ C.L. intervals are indicated with the dark- and light-shaded regions, respectively. The predicted SM value of $\theta_{W Z}$ is indicated by the solid vertical line. 


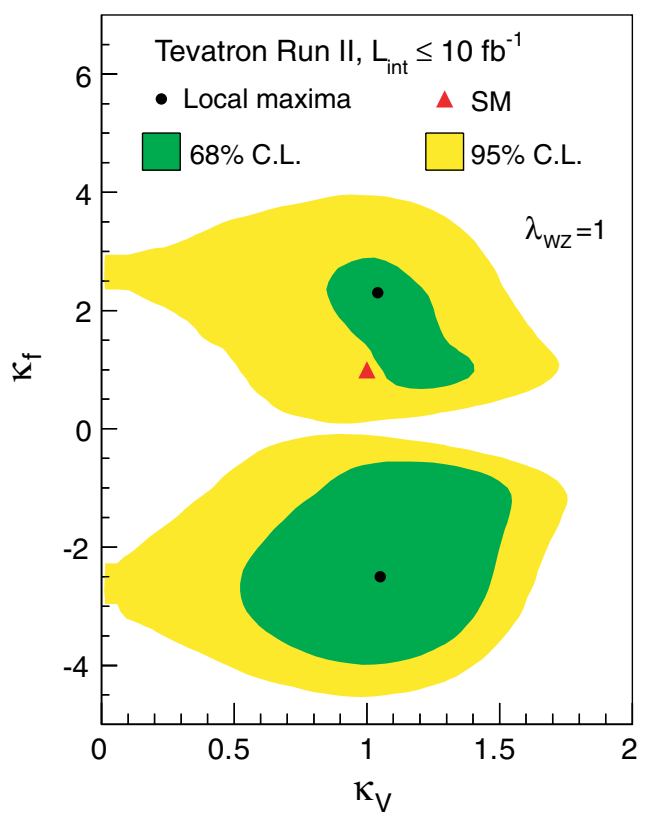

FIG. 22 (color online). Two-dimensional constraints in the $\left(\kappa_{V}, \kappa_{f}\right)$ plane, for the combined Tevatron searches for a SM-like Higgs boson with mass $125 \mathrm{GeV} / c^{2}$ assuming custodial symmetry $\left(\lambda_{W Z}=1\right)$. The points that maximize the local posterior probability densities are marked with dots, and the $68 \%$ and $95 \%$ C.L. intervals are indicated with the dark- and lightshaded regions, respectively. The SM prediction for $\left(\kappa_{V}, \kappa_{f}\right)$ is marked with a triangle.

of the total, while the remaining $74 \%$ of the integral of the posterior density is contained within the $(+,-)$ quadrant.

\section{RESULTS-NON-STANDARD MODEL INTERPRETATIONS}

The mechanism of electroweak symmetry breaking may entail a richer phenomenology than expected in the SM. Natural extensions include the addition of a fourth generation of fermions with masses much larger than those of the three known generations or models with several Higgs bosons or models in which the Higgs boson(s) may have modified couplings. We interpret our Higgs boson search results in models with a sequential fourth generation of fermions (SM4) and in the fermiophobic Higgs model (FHM) described below.

\section{A. Fourth generation interpretation}

With the inclusion of two additional heavy fourth generation quarks in the SM4 [80], the $g g \rightarrow H$ coupling is enhanced by a factor of roughly 3 relative to the SM coupling [81-83]. The partial decay width for $H \rightarrow g g$ is enhanced by the same factor as the production cross section. However, because the $H \rightarrow g g$ decay is mediated by a loop amplitude, the $H \rightarrow W^{+} W^{-}$decay continues to dominate for Higgs boson masses above $135 \mathrm{GeV} / c^{2}$. Since the expected signal yield is larger in the SM4 model than the SM, the sensitivity of CDF's and D0's Higgs boson searches extends to higher masses. For this reason, the upper end of the search range for the relevant channels is raised to $300 \mathrm{GeV} / c^{2}$ for interpretations associated with this model.

Two scenarios for the masses of the fourth generation fermions are considered. In the first, the low-mass scenario, we set the mass of the fourth generation neutrino as $m_{\nu 4}=$ $80 \mathrm{GeV} / c^{2}$ and the mass of the fourth generation charged lepton as $m_{\ell 4}=100 \mathrm{GeV} / c^{2}$, in order to have the maximum impact on the Higgs boson decay branching ratios and to be compatible with the experimental constraint on the mass of an unstable $\nu_{4}$ [84]. In the case that the $\nu_{4}$ is stable or has a lifetime long enough to escape the search presented in Ref. [84], $m_{\nu_{4}}$ could be lighter, modifying the decay branching ratios [85], resulting in weaker mass limits. In our second scenario, the high-mass scenario, we set $m_{\nu 4}=m_{\ell 4}=1 \mathrm{TeV} / c^{2}$, so that the fourth generation leptons do not modify the decay branching ratios of the Higgs boson relative to the SM. In both scenarios, we choose the masses of the quarks to be those of the second scenario in Ref. [83] $\left(m_{d 4}=400 \mathrm{GeV} / c^{2}\right.$ and $m_{u 4}=$ $450 \mathrm{GeV} / c^{2}$ ). The NNLO production cross section calculation of Ref. [83] is used, which is a modified version of the NNLO SM calculation. Previous interpretations of SM Higgs boson searches within the context of a fourth generation of fermions at the Tevatron excluded $131<m_{H}<$ $207 \mathrm{GeV} / c^{2}$ [86]. Similar searches have been performed by the ATLAS [87] and CMS [88] Collaborations, excluding $140<m_{H}<185 \mathrm{GeV} / c^{2}$ and $144<m_{H}<$ $207 \mathrm{GeV} / c^{2}$, respectively. A more recent search by the

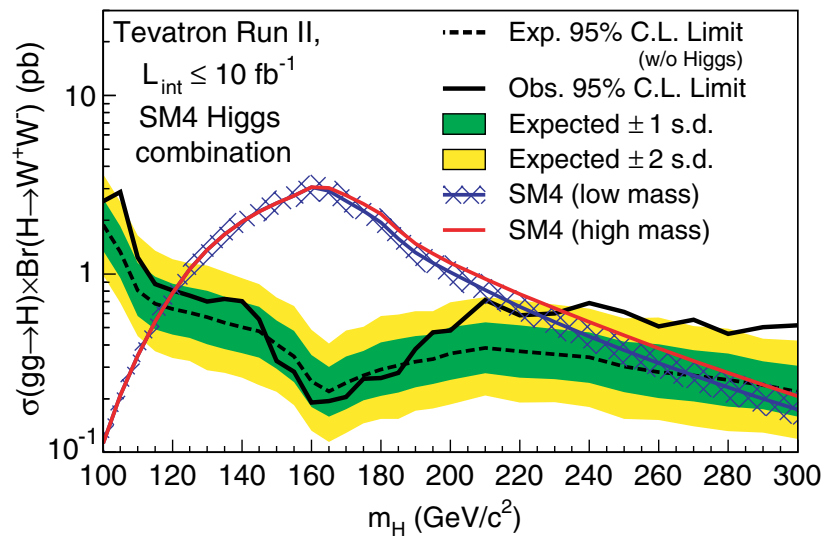

FIG. 23 (color online). Observed and median expected (for the background-only hypothesis) $95 \%$ C.L. Bayesian upper limits on the cross section times branching ratio $\sigma(g g \rightarrow H) \times \mathcal{B}(H \rightarrow$ $W^{+} W^{-}$) from the combination of CDF's and D0's Higgs boson search channels focusing on this production and decay mode. The dark and light-shaded bands indicate, respectively, the 1 and 2 s.d. probability regions in which the limits are expected to fluctuate in the absence of signal. Theoretical predictions for SM4 in the low- and high-mass scenarios are shown with blue and red lines, respectively. The hatched band indicates the theoretical uncertainty associated with the SM4 low-mass scenario. 
CMS Collaboration excluded the mass range $110<m_{H}<$ $600 \mathrm{GeV} / c^{2}$ [89].

We combine our searches for a Higgs boson in the processes $\quad g g \rightarrow H \rightarrow W^{+} W^{-}$and $g g \rightarrow H \rightarrow Z Z$. Limits on the SM4 models and on $\sigma(g g \rightarrow H) \times \mathcal{B}(H \rightarrow$ $W^{+} W^{-}$) are derived. This result is an update of Ref. [86]. The analyses are performed equivalently to the SM searches except that $g g \rightarrow H$ production only is considered for the signal. The MVA classifiers are retrained accordingly and, for the specific case of the D0 $H \rightarrow W^{+} W^{-} \rightarrow$ $\ell^{ \pm} \nu \ell^{\mp} \nu$ channel, the two-jet bin, which is less sensitive to $g g \rightarrow H$ production, is not included.

The branching ratios for $H \rightarrow W^{+} W^{-}$are calculated using HDECAY [26] modified to include fourth generation

TABLE VIII. Observed and median expected (for the background-only hypothesis) $95 \%$ C.L. upper limits on the cross section times branching ratio $\sigma(g g \rightarrow H) \times \mathcal{B}\left(H \rightarrow W^{+} W^{-}\right)$ from the combination of CDF's and D0's Higgs boson search channels focusing on this production and decay mode, obtained using the Bayesian and $\mathrm{CL}_{\mathrm{s}}$ methods.

\begin{tabular}{|c|c|c|c|c|}
\hline \multirow[b]{2}{*}{$m_{H}\left(\mathrm{GeV} / c^{2}\right)$} & \multicolumn{2}{|c|}{ Bayesian } & \multicolumn{2}{|c|}{$\mathrm{CL}_{\mathrm{s}}$} \\
\hline & $\begin{array}{l}\text { Observed } \\
\text { limit }(\mathrm{pb})\end{array}$ & $\begin{array}{l}\text { Expected } \\
\text { limit }(p b)\end{array}$ & $\begin{array}{l}\text { Observed } \\
\text { limit }(\mathrm{pb})\end{array}$ & $\begin{array}{l}\text { Expected } \\
\text { limit }(\mathrm{pb})\end{array}$ \\
\hline 100 & 2.56 & 1.89 & 2.58 & 1.87 \\
\hline 105 & 2.87 & 1.33 & 2.62 & 1.32 \\
\hline 110 & 1.24 & 0.81 & 1.27 & 0.82 \\
\hline 115 & 0.88 & 0.68 & 0.90 & 0.70 \\
\hline 120 & 0.81 & 0.63 & 0.81 & 0.66 \\
\hline 125 & 0.75 & 0.61 & 0.77 & 0.61 \\
\hline 130 & 0.70 & 0.57 & 0.70 & 0.58 \\
\hline 135 & 0.72 & 0.53 & 0.75 & 0.54 \\
\hline 140 & 0.70 & 0.50 & 0.72 & 0.52 \\
\hline 145 & 0.56 & 0.48 & 0.55 & 0.48 \\
\hline 150 & 0.33 & 0.40 & 0.32 & 0.42 \\
\hline 155 & 0.28 & 0.34 & 0.30 & 0.35 \\
\hline 160 & 0.19 & 0.25 & 0.20 & 0.26 \\
\hline 165 & 0.19 & 0.22 & 0.20 & 0.22 \\
\hline 170 & 0.20 & 0.24 & 0.21 & 0.25 \\
\hline 175 & 0.26 & 0.27 & 0.26 & 0.27 \\
\hline 180 & 0.26 & 0.29 & 0.26 & 0.29 \\
\hline 185 & 0.28 & 0.31 & 0.29 & 0.31 \\
\hline 190 & 0.38 & 0.32 & 0.40 & 0.33 \\
\hline 195 & 0.47 & 0.33 & 0.47 & 0.34 \\
\hline 200 & 0.48 & 0.36 & 0.49 & 0.37 \\
\hline 210 & 0.71 & 0.38 & 0.73 & 0.39 \\
\hline 220 & 0.59 & 0.37 & 0.60 & 0.37 \\
\hline 230 & 0.60 & 0.36 & 0.61 & 0.36 \\
\hline 240 & 0.69 & 0.34 & 0.69 & 0.34 \\
\hline 250 & 0.61 & 0.30 & 0.60 & 0.30 \\
\hline 260 & 0.51 & 0.28 & 0.49 & 0.29 \\
\hline 270 & 0.55 & 0.27 & 0.56 & 0.27 \\
\hline 280 & 0.46 & 0.25 & 0.47 & 0.25 \\
\hline 290 & 0.50 & 0.24 & 0.48 & 0.24 \\
\hline 300 & 0.52 & 0.22 & 0.50 & 0.22 \\
\hline
\end{tabular}

fermions [82]. To include the $g g \rightarrow H \rightarrow Z Z$ searches, we assume the SM value for $\mathcal{B}\left(H \rightarrow W^{+} W^{-}\right) / \mathcal{B}(H \rightarrow Z Z)$. In setting limits on $\sigma(g g \rightarrow H) \times \mathcal{B}\left(H \rightarrow W^{+} W^{-}\right)$, the $g g \rightarrow H \rightarrow Z Z$ process is included assuming that its signal yield scales equivalently to that from the $g g \rightarrow H \rightarrow$ $W^{+} W^{-}$channel.

When setting limits on $\sigma(g g \rightarrow H) \times \mathcal{B}\left(H \rightarrow W^{+} W^{-}\right)$, the theoretical uncertainty on the prediction of $\sigma(g g \rightarrow H) \times \mathcal{B}\left(H \rightarrow W^{+} W^{-}\right)$is not included since these limits are independent of the predictions. However, when setting limits on $m_{H}$ in the context of fourth generation models, uncertainties on the theoretical predictions are included as described for the SM searches.

The combined limits on $\sigma(g g \rightarrow H) \times \mathcal{B}\left(H \rightarrow W^{+} W^{-}\right)$ obtained using the Bayesian method are shown in Fig. 23 along with the theory predictions for fourth generation models in the low- and high-mass scenarios. Limits obtained using both the Bayesian and $\mathrm{CL}_{\mathrm{s}}$ methods are listed as a function of Higgs boson mass in Table VIII. A broad, moderate excess above the background expectation is seen for masses above $200 \mathrm{GeV} / c^{2}$.

Production limits obtained for the two SM4 scenarios using the Bayesian method are shown in Fig. 24. The limits are presented as ratios relative to SM4 low-mass scenario predictions as a function of the Higgs boson mass. In the low-mass scenario, which gives the smaller excluded mass range, a SM-like Higgs boson with a mass in the range $121-225 \mathrm{GeV} / c^{2}$ is excluded at the $95 \%$ C.L.

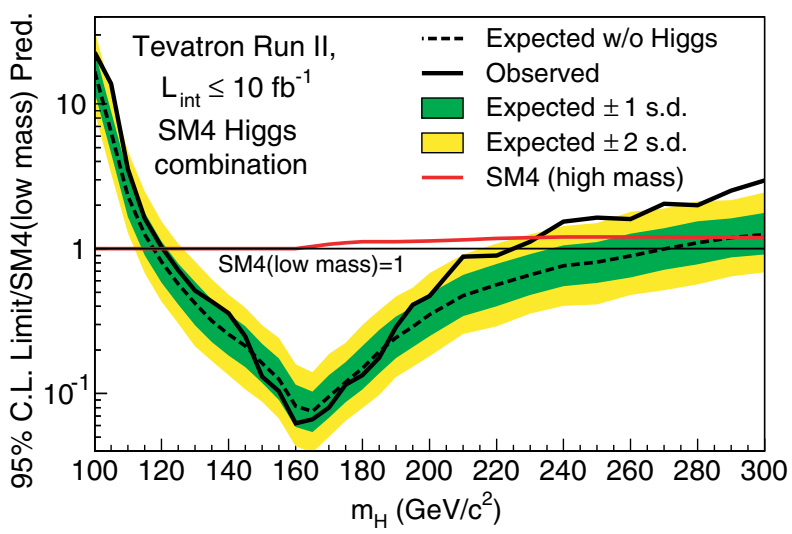

FIG. 24 (color online). Observed and median expected (for the background-only hypothesis) $95 \%$ C.L. Bayesian upper production limits expressed as multiples of the SM4 (low-mass scenario) cross section as a function of Higgs boson mass from the combination of CDF's and D0's Higgs boson searches focusing on the $g g \rightarrow H$ production and $H \rightarrow W^{+} W^{-}$decay modes. Uncertainties associated with theoretical cross section and branching ratio predictions are incorporated in the limit. The dark- and light-shaded bands indicate, respectively, the 1 and 2 s.d. probability regions in which the limits are expected to fluctuate in the absence of signal. The red line shows the prediction for the signal rate in the high-mass scenario, divided by that of the low-mass scenario. 
The expected excluded mass range is $118-270 \mathrm{GeV} / c^{2}$. In the high-mass scenario, the mass range $121-232 \mathrm{GeV} / c^{2}$ is excluded, with an expected excluded mass range of $118-290 \mathrm{GeV} / c^{2}$.

\section{B. Fermiophobic interpretation}

In the FHM, the lightest Higgs boson does not couple to fermions at tree level, but aside from this one difference, its behavior is indistinguishable from that of the SM Higgs boson. In the FHM, the production of Higgs bosons, $H_{f}$, at hadron colliders via the process $g g \rightarrow H_{f}$ is suppressed to a negligible rate and is ignored in the context of this interpretation. The associated production mechanisms $p \bar{p} \rightarrow W H_{f}+X$ and $p \bar{p} \rightarrow Z H_{f}+X$, as well as the vector-boson-fusion (VBF) processes $q \bar{q} \rightarrow q^{\prime} \bar{q}^{\prime} H_{f}$, remain nearly unchanged relative to the corresponding processes in the SM. Thus, the corresponding SM cross sections and associated uncertainties described previously are also used here. In the FHM, direct decays to fermions are forbidden; the decays to $W^{+} W^{-}, \gamma \gamma, Z Z$, and $Z \gamma$ account for nearly the entire decay width. For the mass range under investigation the $W^{+} W^{-}$decay mode has the largest branching fraction. The branching fraction $\mathcal{B}\left(H_{f} \rightarrow \gamma \gamma\right)$ is greatly enhanced over $\mathcal{B}\left(H_{\mathrm{SM}} \rightarrow \gamma \gamma\right)$ for

TABLE IX. Ratios of observed and median expected (for the background-only hypothesis) $95 \%$ C.L. upper limits on the production rate of a fermiophobic Higgs boson relative to the FHM prediction as a function of the Higgs boson mass for the combination of CDF's and D0's searches, obtained using the Bayesian and $\mathrm{CL}_{\mathrm{s}}$ methods.

\begin{tabular}{lllll}
\hline \hline & \multicolumn{3}{c}{ Bayesian } & \multicolumn{2}{c}{$\mathrm{CL}_{\mathrm{s}}$} \\
$m_{H_{f}}\left(\mathrm{GeV} / c^{2}\right)$ & $R_{95}^{\text {obs }}$ & $R_{95}^{\text {exp }}$ & $R_{95}^{\text {obs }}$ & $R_{95}^{\text {exp }}$ \\
\hline 100 & 0.21 & 0.13 & 0.21 & 0.13 \\
105 & 0.36 & 0.22 & 0.37 & 0.23 \\
110 & 0.40 & 0.37 & 0.36 & 0.37 \\
115 & 0.95 & 0.54 & 0.88 & 0.53 \\
120 & 1.13 & 0.69 & 1.06 & 0.68 \\
125 & 1.41 & 0.83 & 1.44 & 0.81 \\
130 & 1.21 & 0.91 & 1.06 & 0.90 \\
135 & 1.26 & 1.00 & 1.16 & 0.97 \\
140 & 1.65 & 1.11 & 1.48 & 1.06 \\
145 & 1.47 & 1.15 & 1.30 & 1.13 \\
150 & 1.33 & 1.21 & 1.19 & 1.17 \\
155 & 1.30 & 1.19 & 1.17 & 1.18 \\
160 & 1.20 & 1.17 & 1.11 & 1.14 \\
165 & 0.98 & 1.17 & 0.94 & 1.11 \\
170 & 1.49 & 1.31 & 1.35 & 1.26 \\
175 & 1.96 & 1.48 & 1.76 & 1.43 \\
180 & 2.34 & 1.72 & 2.04 & 1.60 \\
185 & 3.13 & 1.96 & 2.58 & 1.93 \\
190 & 3.75 & 2.36 & 3.24 & 2.32 \\
195 & 4.58 & 2.62 & 3.92 & 2.54 \\
200 & 5.43 & 2.85 & 4.64 & 2.77 \\
\hline \hline
\end{tabular}

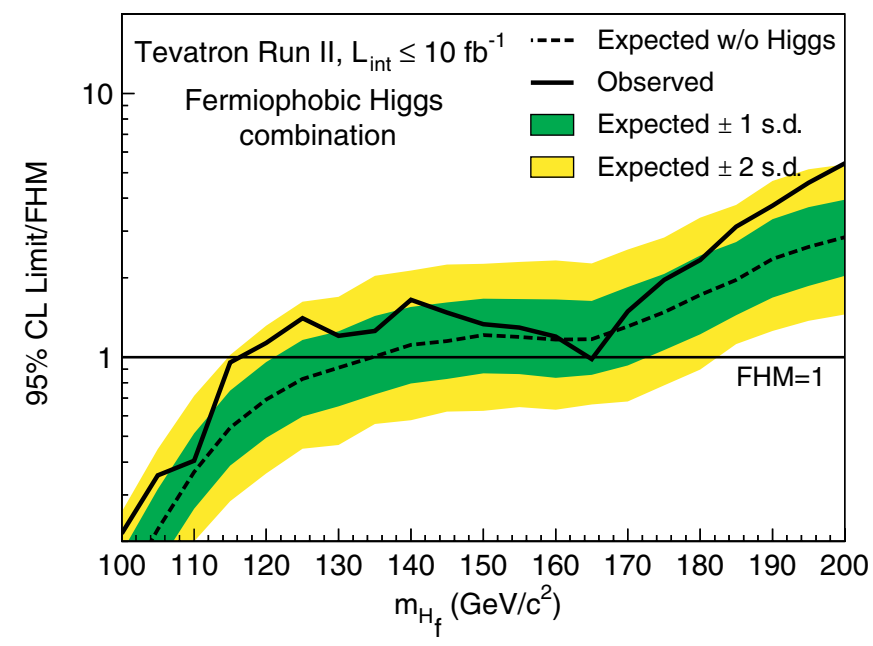

FIG. 25 (color online). Observed and median expected (for the background-only hypothesis) $95 \%$ C.L. Bayesian upper production limits expressed as multiples of the FHM cross section as a function of Higgs boson mass for the combined CDF and D0 searches. The dark- and light-shaded bands indicate, respectively, the 1 and 2 s.d. probability regions in which the limits are expected to fluctuate in the absence of signal.

all $m_{H}$, and the clean signature and excellent mass resolution of this channel provide most of the search sensitivity for $m_{H_{f}}<120 \mathrm{GeV} / c^{2}$. The analyses combined here seek Higgs boson decays to $W^{+} W^{-}, \gamma \gamma$, and ZZ. Previous searches for a fermiophobic Higgs boson at the Tevatron excluded signals with masses smaller than $119 \mathrm{GeV} / c^{2}$ [90]; the expected exclusion was also $m_{H_{f}}<119 \mathrm{GeV} / c^{2}$. The ATLAS and CMS Collaborations excluded $m_{H_{f}}$ in the ranges $110.0-118.0 \mathrm{GeV} / c^{2}$ and $119.5-121.0 \mathrm{GeV} / c^{2}$ using diphoton final states [91] and in the range $110-194 \mathrm{GeV} / c^{2}$ by combining multiple final states [92].

The SM $H \rightarrow \gamma \gamma$ analyses are reoptimized as the kinematic distributions of the Higgs bosons, their decay products, and the particles produced in association with the Higgs bosons differ between the FHM and the SM. Events contain either an associated $W$ or $Z$ boson, or recoiling quark jets in the case of VBF, and thus the transverse momentum $\left(p_{T}\right)$ of the Higgs boson is on average greater than it is in the SM. The analyses combined here update previous searches for the Higgs boson in the FHM $[93,94]$. Similarly, SM searches in $H \rightarrow W^{+} W^{-}$channels cannot be interpreted directly in the FHM due to the different mixture of production modes. Signal contributions from $g g \rightarrow H_{f}$ production to the MVA discriminant distributions are ignored, and the remaining contributions from other production mechanisms are scaled by the ratio of branching ratio predictions $\mathcal{B}\left(H_{f} \rightarrow V V\right) / \mathcal{B}\left(H_{\mathrm{SM}} \rightarrow V V\right)$. The existing subdivision of channels based on the number of reconstructed jets accompanying the leptons and missing transverse energy in the event naturally optimizes the search within the FHM interpretation. Hence, the development of a 
separate set of analysis channels as in the case of $H_{f} \rightarrow \gamma \gamma$ is not required, though the MVAs are retrained.

The combined limits on Higgs boson production normalized to FHM predictions obtained from both the Bayesian and $\mathrm{CL}_{\mathrm{s}}$ methods are listed in Table IX as a function of Higgs boson mass. The expected limits assume no Higgs boson production. The limits obtained using the Bayesian method are shown in Fig. 25. Fermiophobic Higgs bosons in the mass range $100-116 \mathrm{GeV} / c^{2}$ are excluded at the $95 \%$ C.L.; the expected excluded mass range is $100-135 \mathrm{GeV} / c^{2}$.

\section{CONCLUSIONS}

The search for the standard model Higgs boson at the Tevatron is challenging due to the small expected signal and the need to accurately model large background contributions. We have developed advanced tools to search for the Higgs boson in the leading production and decay modes predicted by the SM and control the impact of systematic uncertainties using constraints from the observed data. We have combined searches by the CDF and D0 Collaborations for the standard model Higgs boson in the mass range $90-200 \mathrm{GeV} / c^{2}$ using Tevatron $p \bar{p}$ collision data corresponding to up to $10 \mathrm{fb}^{-1}$ of integrated luminosity collected at $\sqrt{s}=1.96 \mathrm{TeV}$. The results of searches focusing on the $H \rightarrow b \bar{b}, H \rightarrow$ $W^{+} W^{-}, \quad H \rightarrow Z Z, \quad H \rightarrow \tau^{+} \tau^{-}$, and $H \rightarrow \gamma \gamma$ decay modes are included in the combination. The results are also interpreted in fermiophobic and fourth generation models. Fermiophobic Higgs bosons in the mass range $100-116 \mathrm{GeV} / c^{2}$ are excluded at the $95 \%$ C.L., and a SM-like Higgs boson in the presence of a fourth sequential generation of fermions is excluded in the mass range $121-225 \mathrm{GeV} / c^{2}$ at the $95 \%$ C.L. The SM Higgs boson is excluded, at the $95 \%$ C.L., from 90 to $109 \mathrm{GeV} / c^{2}$, and from 149 to $182 \mathrm{GeV} / c^{2}$. The expected exclusion regions in the absence of signal are $90-120 \mathrm{GeV} / c^{2}$ and $140-184 \mathrm{GeV} / c^{2}$. The results of the $H \rightarrow b \bar{b}$ searches were validated through a measurement of the diboson $(W Z+Z Z)$ production cross section using the same data samples and analysis techniques, treating those diboson processes as signal. The resulting diboson cross-section measurement is in agreement with the SM prediction. We observe a significant excess of events in the mass range between 115 and $140 \mathrm{GeV} / c^{2}$. The local significance at $m_{H}=125 \mathrm{GeV} / c^{2}$ corresponds to 3.0 standard deviations, with a median expected significance, assuming the SM Higgs boson is present at $m_{H}=$ $125 \mathrm{GeV} / c^{2}$, of 1.9 standard deviations, with a best-fit signal strength of $1.44_{-0.56}^{+0.59}$ times the SM expectation. We also separately combined searches focusing on the $H \rightarrow b \bar{b}, H \rightarrow W^{+} W^{-}, H \rightarrow \tau^{+} \tau^{-}$, and $H \rightarrow \gamma \gamma$ decay modes. The observed best-fit signal strengths obtained from each of these combinations are consistent with the expectations for a SM Higgs boson at $m_{H}=$ $125 \mathrm{GeV} / c^{2}$. We performed tests of the compatibility of the observed excess with the expectations for the couplings of a SM Higgs boson and saw no significant deviations.

\section{ACKNOWLEDGMENTS}

We thank the Fermilab staff and technical staffs of the participating institutions for their vital contributions. We acknowledge support from the DOE and NSF (USA), ARC (Australia), CNPq, FAPERJ, FAPESP and FUNDUNESP (Brazil), NSERC (Canada), NSC, CAS and CNSF (China), Colciencias (Colombia), MSMT and GACR (Czech Republic), the Academy of Finland, CEA and CNRS/IN2P3 (France), BMBF and DFG (Germany), DAE and DST (India), SFI (Ireland), INFN (Italy), MEXT (Japan), the Korean World Class University Program and NRF (Korea), CONACyT (Mexico), FOM (Netherlands), MON, NRC KI and RFBR (Russia), the Slovak R\&D Agency, the Ministerio de Ciencia e Innovación, and Programa Consolider-Ingenio 2010 (Spain), The Swedish Research Council (Sweden), SNSF (Switzerland), STFC and the Royal Society (United Kingdom), the A.P. Sloan Foundation (USA), and the EU community Marie Curie Fellowship Contract No. 302103.
[1] S. L. Glashow, Nucl. Phys. 22, 579 (1961); S. Weinberg, Phys. Rev. Lett. 19, 1264 (1967); A. Salam, Elementary Particle Theory, edited by N. Svartholm (Almqvist \& Wiksell, Stockholm, 1968), p. 367.

[2] F. Englert and R. Brout, Phys. Rev. Lett. 13, 321 (1964); P. W. Higgs, Phys. Rev. Lett. 13, 508 (1964); G. S. Guralnik, C. R. Hagen, and T.W. B. Kibble, Phys. Rev. Lett. 13, 585 (1964); P. W. Higgs, Phys. Rev. 145, 1156 (1966).
[3] T. Aaltonen et al. (CDF and D0 Collaborations), arXiv:1204.0042.

[4] T. Aaltonen et al. (CDF and D0 Collaborations), Phys. Rev. D 86, 092003 (2012).

[5] ALEPH, CDF, D0, DELPHI, L3, OPAL, and SLD Collaborations, LEP Electroweak Working Group, the Tevatron Electroweak Working Group, and SLD Electroweak and Heavy Flavour Working Groups, arXiv:1012.2367v2. The most recent values from March 
2012, as quoted, are available from http://lepewwg .web.cern.ch/LEPEWWG/.

[6] In this article, C.L. denotes confidence level for frequentist and modified frequentist results, and credibility level for Bayesian results.

[7] ALEPH, DELPHI, L3, and OPAL Collaborations, and LEP Working Group for Higgs Boson Searches, Phys. Lett. B 565, 61 (2003).

[8] G. Aad et al. (ATLAS Collaboration), Phys. Lett. B 716, 1 (2012).

[9] S. Chatrchyan et al. (CMS Collaboration), Phys. Lett. B 716, 30 (2012).

[10] G. Aad et al. (ATLAS Collaboration), Phys. Lett. B 718, 369 (2012).

[11] S. Chatrchyan et al. (CMS Collaboration), Phys. Lett. B 710, 284 (2012).

[12] T. Aaltonen et al. (CDF and D0 Collaborations), Phys. Rev. Lett. 109, 071804 (2012).

[13] T. Aaltonen et al. (CDF and D0 Collaborations), Phys. Rev. Lett. 104, 061802 (2010).

[14] T. Aaltonen et al. (CDF Collaboration), preceding article, Phys. Rev. D. 88, 052013 (2013).

[15] V. M. Abazov et al. (D0 Collaboration), this issue, Phys. Rev. D. 88, 052011 (2013).

[16] T. Sjöstrand, S. Mrenna, and P. Skands, J. High Energy Phys. 05 (2006) 026. We use PYTHIA version 6.216 to generate the Higgs boson signals.

[17] H. L. Lai, J. Huston, S. Kuhlmann, J. Morfin, F. Olness, J. F. Owens, J. Pumplin, and W. K. Tung, Eur. Phys. J. C 12, 375 (2000); J. Pumplin, D. R. Stump, J. Huston, H-L. Lai, P. Nadolsky, and W-K. Tung, J. High Energy Phys. 07 (2002) 012.

[18] C. Anastasiou, R. Boughezal, and F. Petriello, J. High Energy Phys. 04 (2009) 003.

[19] D. de Florian and M. Grazzini, Phys. Lett. B 674, 291 (2009).

[20] J. Baglio and A. Djouadi, J. High Energy Phys. 10 (2010) 064; O. Brein, R. V. Harlander, M. Weisemann, and T. Zirke, Eur. Phys. J. C 72, 1868 (2012).

[21] P. Bolzoni, F. Maltoni, S.-O. Moch, and M. Zaro, Phys. Rev. Lett. 105, 011801 (2010).

[22] M. Ciccolini, A. Denner, and S. Dittmaier, Phys. Rev. Lett. 99, 161803 (2007); Phys. Rev. D 77, 013002 (2008).

[23] W. Beenakker, S. Dittmaier, M. Krämer, B. Plümper, M. Spira, and P. M. Zerwas, Phys. Rev. Lett. 87, 201805 (2001); L. Reina and S. Dawson, Phys. Rev. Lett. 87, 201804 (2001).

[24] A. D. Martin, W. J. Stirling, R. S. Thorne, and G. Watt, Eur. Phys. J. C 63, 189 (2009).

[25] S. Dittmaier et al. (LHC Higgs Cross Section Working Group), arXiv:1201.3084.

[26] A. Djouadi, J. Kalinowski, and M. Spira, Comput. Phys. Commun. 108, 56 (1998). We use HDECAY Version 3.53 .

[27] A. Bredenstein, A. Denner, S. Dittmaier, and M. M. Weber, Phys. Rev. D 74, 013004 (2006); A. Bredenstein, A. Denner, S. Dittmaier, A. Mück, and M. M. Weber, J. High Energy Phys. 02 (2007) 080.

[28] G. Bozzi, S. Catani, D. de Florian, and M. Grazzini, Phys. Lett. B 564, 65 (2003); Nucl. Phys. B737, 73 (2006).
[29] M. Mangano, M. Moretti, F. Piccinini, R. Pittau, and A. Polosa, J. High Energy Phys. 07 (2003) 001.

[30] S. Frixione and B. R. Webber, J. High Energy Phys. 06 (2002) 029.

[31] G. Corcella, I. G. Knowles, G. Marchesini, S. Moretti, K. Odagiri, P. Richardson, M. H. Seymour, and B. R. Webber, J. High Energy Phys. 01, (2001) 010.

[32] E. Boos, V. Bunichev, M. Dubinin, L. Dudko, V. Ilyin, A. Kryukov, V. Edneral, V. Savrin, A. Semenov, and A. Sherstnev, Nucl. Instrum. Methods Phys. Res., Sect. A 534, 250 (2004); E. E. Boos, V.E. Bunichev, L. V. Dudko, V. I. Savrin, and A. V. Sherstnev, Phys. At. Nucl. 69, 1317 (2006).

[33] J. M. Campbell and R. K. Ellis, Phys. Rev. D 60, 113006 (1999).

[34] U. Langenfeld, S. Moch, and P. Uwer, Phys. Rev. D 80, 054009 (2009).

[35] N. Kidonakis, Phys. Rev. D 74, 114012 (2006).

[36] R. Hamberg, W. L. van Neerven, and T. Matsuura, Nucl. Phys. B359, 343 (1991); B644, 403(E) (2002).

[37] A heavy-flavor jet is a reconstructed cluster of calorimeter energies associated with particles produced in the hadronization and decay of a bottom or charm quark.

[38] A $b$-tagged jet is one identified to have originated from the decay of a heavy flavor quark.

[39] R. Brun and F. Carminati, CERN Program Library Long Writeup W5013, 1993 (unpublished).

[40] D. Acosta et al. (CDF Collaboration), Phys. Rev. D 71, 032001 (2005); A. Abulencia et al. (CDF Collaboration), J. Phys. G 34, 2457 (2007).

[41] V. M. Abazov et al. (D0 Collaboration), Nucl. Instrum. Methods Phys. Res., Sect. A 565, 463 (2006); M. Abolins et al., Nucl. Instrum. Methods Phys. Res., Sect. A 584, 75 (2008); R. Angstadt et al., Nucl. Instrum. Methods Phys. Res., Sect. A 622, 298 (2010).

[42] T. Aaltonen et al. (CDF Collaboration), Phys. Rev. Lett. 109, 111804 (2012).

[43] T. Aaltonen et al. (CDF Collaboration), Phys. Rev. D 87, 052008 (2013).

[44] T. Aaltonen et al. (CDF Collaboration), Phys. Rev. Lett. 109, 111803 (2012).

[45] T. Aaltonen et al. (CDF Collaboration), J. High Energy Phys. 02 (2013) 004.

[46] T. Aaltonen et al. (CDF Collaboration), Phys. Rev. Lett. 109, 181802 (2012).

[47] T. Aaltonen et al. (CDF Collaboration), this issue, Phys. Rev. D 88, 052012 (2013).

[48] T. Aaltonen et al. (CDF Collaboration), Phys. Rev. Lett. 108, 181804 (2012).

[49] T. Aaltonen et al. (CDF Collaboration), Phys. Lett. B 717, 173 (2012).

[50] T. Aaltonen et al. (CDF Collaboration), Phys. Rev. D 86, 072012 (2012).

[51] V. M. Abazov et al. (D0 Collaboration), Phys. Rev. Lett. 109, 121804 (2012).

[52] V. M. Abazov et al. (D0 Collaboration), this issue, Phys. Rev. D. 88, 052008 (2013).

[53] V. M. Abazov et al. (D0 Collaboration), Phys. Lett. B 716, 285 (2012)

[54] V. M. Abazov et al. (D0 Collaboration), Phys. Rev. Lett. 109, 121803 (2012). 
[55] V. M. Abazov et al. (D0 Collaboration), this issue, Phys. Rev. D. 88, 052010 (2013).

[56] V. M. Abazov et al. (D0 Collaboration), this issue, Phys. Rev. D. 88, 052006 (2013).

[57] V. M. Abazov et al. (D0 Collaboration), Phys. Lett. B 714, 237 (2012)

[58] V. M. Abazov et al. (D0 Collaboration), this issue, Phys. Rev. D. 88, 052009 (2013).

[59] V. M. Abazov et al. (D0 Collaboration), this issue, Phys. Rev. D. 88, 052005 (2013).

[60] V. M. Abazov et al. (D0 Collaboration), this issue, Phys. Rev. D. 88, 052007 (2013).

[61] For a recent review, see P. C. Bhat, Annu. Rev. Nucl. Part. Sci. 61, 281 (2011). The specific details of the MVA for each analysis are described in the respective references.

[62] J. Freeman, T. Junk, M. Kirby, Y. Oksuzian, T. J. Phillips, F. D. Snider, M. Trovato, J. Vizan, and W. M. Yao, Nucl. Instrum. Methods Phys. Res., Sect. A 697, 64 (2013); D. Acosta et al. (CDF Collaboration), Phys. Rev. D 71, 052003 (2005); A. Abulencia et al. (CDF Collaboration), Phys. Rev. D 74, 072006 (2006).

[63] V. M. Abazov et al., Nucl. Instrum. Methods Phys. Res., Sect. A 620, 490 (2010).

[64] Statistics in K. Nakamura et al. (Particle Data Group), J. Phys. G 37, 075021 (2010).

[65] W. Fisher, Report No. FERMILAB-TM-2386-E (2006).

[66] T. Junk, Nucl. Instrum. Methods Phys. Res., Sect. A 434, 435 (1999); A. L. Read, J. Phys. G 28, 2693 (2002).

[67] S. Alekhin et al. (PDF4LHC Working Group), arXiv:1101.0536; M. Botje et al. (PDF4LHC Working Group), arXiv:1101.0538.

[68] C. Anastasiou, G. Dissertori, M. Grazzini, F. Stöckli, and B. R. Webber, J. High Energy Phys. 08 (2009) 099.

[69] J. Baglio and A. Djouadi, J. High Energy Phys. 03 (2011) 055.

[70] I. W. Stewart and F. J. Tackmann, Phys. Rev. D 85, 034011 (2012).

[71] J. M. Campbell, R. K. Ellis, and C. Williams, Phys. Rev. D 81, 074023 (2010).

[72] K. Nakamura et al. (Particle Data Group), J. Phys. G 37, 075021 (2010).

[73] T. Aaltonen et al. (CDF Collaboration), Phys. Rev. Lett. 104, 201801 (2010); 108, 101801 (2012); D. Acosta et al., Phys. Rev. D 86, 031104(R) (2012).
[74] T. Aaltonen et al. (CDF Collaboration), Phys. Rev. Lett. 109, 111805 (2012).

[75] As discussed later, a particular decay mode defined by an experimental signature as done here may be an admixture of several decay modes, though dominated by the one denoted.

[76] J. R. Espinosa, C. Grojean, M. Mühlleitner, and M. Trott, J. High Energy Phys. 12 (2012) 045.

[77] A. David et al. (LHC Higgs Cross Section Working Group), arXiv:1209.0040.

[78] M. Spira, A. Djouadi, D. Graudenz, and P. M. Zerwas, Nucl. Phys. B453, 17 (1995).

[79] P. Sikivie, L. Susskind, M. B.Voloshin, and V. Zakarov, Nucl. Phys. B173, 189 (1980).

[80] B. Holdom, W. S. Hou, T. Hurth, M. L. Mangano, S. Sultansoy, and G. Unel, PMC Phys. A 3, 4 (2009).

[81] E. Arik, O. Cakir, S. A. Cetin, and S. Sultansoy, Acta Phys. Pol. B 37, 2839 (2006).

[82] G. D. Kribs, T. Plehn, M. Spannowsky, and T. M.P. Tait, Phys. Rev. D 76, 075016 (2007).

[83] C. Anastasiou, R. Boughezal, and E. Furlan, J. High Energy Phys. 06 (2010) 101.

[84] P. Achard et al. (L3 Collaboration), Phys. Lett. B 517, 75 (2001).

[85] K. Belotsky, D. Fargion, M. Khlopov, R. Konoplich, and K. Shibaev, Phys. Rev. D 68, 054027 (2003).

[86] T. Aaltonen et al. (CDF and D0 Collaborations), Phys. Rev. D 82, 011102 (2010).

[87] G. Aad et al. (ATLAS Collaboration), Eur. Phys. J. C 71, 1728 (2011).

[88] S. Chatrchyan et al. (CMS Collaboration), Phys. Lett. B 699, 25 (2011).

[89] S. Chatrchyan et al. (CMS Collaboration), Phys. Lett. B 725, 36 (2013).

[90] T. Aaltonen et al. (CDF and D0 Collaborations), arXiv:1109.0576.

[91] G. Aad et al. (ATLAS Collaboration), Eur. Phys. J. C 72, 2157 (2012).

[92] S. Chatrchyan et al. (CMS Collaboration), J. High Energy Phys. 09 (2012) 111.

[93] T. Aaltonen et al. (CDF Collaboration), Phys. Rev. Lett. 103, 061803 (2009).

[94] V. M. Abazov et al. (D0 Collaboration), Phys. Rev. Lett. 102, 231801 (2009). 